\title{
Funnel Plots for Assessing Institutional Performance
}

\author{
Amy Jennings
}

\author{
A thesis \\ submitted to the Victoria University of Wellington \\ in fulfilment of the \\ requirements for the degree of \\ Master of Science in Statistics \\ Victoria University of Wellington \\ 2013
}





\begin{abstract}
This thesis presents a comparison of maternal outcomes for births in New Zealand District Health Boards (DHBs). This is carried out through analysis of the National Minimum Dataset collected by the Ministry of Health for 2007.
\end{abstract}

The outcome compared is postpartum haemorrhage (PPH) the results are displayed using funnel plots, a useful tool for displaying unbiased information on performance outcomes when comparing institutions.

Exploration of the data found that there are differences in the demographics, maternal and birth characteristics among DHBs. The rates of PPH are different and the population mixes are made up of a range of different proportions of ethnic groups, ages and deprivation indexes. The exploratory analysis found that a large number of factors are associated with PPH. And that birth weight, parity and gestation had a large number of missing observations. These factors are not missing at random and require imputing prior to constructing the funnel plots.

Results show that there is divergence amongst DHBs in the postpartum haemorrhage rate. First a raw PPH rate was plotted and the results indicated there were differences among DHBs. As there are many potential predictors for $\mathrm{PPH}$ a logistic regression model was applied to find the most important factors related to PPH. This allows us to apply an adjusted rate for the funnel plot. The risk adjusted funnel plot also indicated differences among DHBs. 
Two approaches are taken to account for the overdispersion. A winsorised estimate and a winsorised estimate with a random effects term are applied to the data. The approaches produced different results. The winsorised estimate widened the control limits and the random effects term narrowed the control limits. All four plots identified an extreme outlier and this was later removed from the analysis and the winsorisation funnel plots were rerun. The influential outlier made a difference and from this we can concluded that 2 out 20 DHBs lie outside the $95 \%$ control limits. These two DHBs could be stated as having a very low rate of PPH. 


\section{Acknowledgments}

Firstly I would like to thank my supervisors Nokuthaba Sibanda and Ivy Liu for their help, advice, support and motivation through this long journey and for being understanding and patient with me through out this challenge I set upon myself.

I would like to acknowledge Lisa Woods and Franzsika Broell for cleaning and preparing the dataset that I have work with.

Thank you to my work colleagues for all your support and encouragement through the past two years, this journey could of not been complete without you.

I would like to thank Clive for helping me get started with writing and your general support, motivation and drive through out the last two years.

I would like to thank my friends and family for all your support, encouragement, positivity and continuously telling me I can do it! Thank you to Dr Kirsten Wadsworth for your fantastic proof reading and editing.

And lastly I would like to thank my partner Llewe, my Super Hero! For my constant demanding for coffee, proof reading, layout, editing and bringing me chocolate. And for being supportive, encouraging, devoted, loving and dedicated to me and my thesis journey over these past few years. 


\section{Contents}

1 Introduction $\quad \mathbf{1}$

1.1 Background .................... 1

1.2 Structure of this Thesis . . . . . . . . . . . . . . . 4

1.3 Research Objectives . . . . . . . . . . . . . . . . 5

1.4 Postpartum Haemorrhage . . . . . . . . . . . . . 5

2 Literature Review $\quad 9$

2.1 Literature Review on Funnel Plots . . . . . . . . . . . . 9

2.2 New Zealand Research . . . . . . . . . . . . . . . . . . . 13

3 Data Description 17

3.1 Data Collection Procedure . . . . . . . . . . . . . 17

3.2 Coding of Ethnicity . . . . . . . . . . . . . . . . . . 19

3.3 Data Quality Issues . . . . . . . . . . . . . . . . . 20

3.4 Study Dataset . . . . . . . . . . . . . . . . . 21

3.5 Data Cleaning . . . . . . . . . . . . . . . . . . 21

3.6 Derived Variables . . . . . . . . . . . . . . . . . . 22

4 Statistical Methods $\quad 27$

4.1 Exploratory Data Analysis . . . . . . . . . . . . . . . 27

4.2 Funnel Plots . . . . . . . . . . . . . . . . . . . 28

4.2.1 Unadjusted Funnel Plot . . . . . . . . . . . . . . . . 30

4.2.2 Risk Adjusted Funnel Plot . . . . . . . . . . . . . . . . 31 
4.2.3 Winsorised Funnel Plots . . . . . . . . . . . . . 32

4.2.4 Winsorisation using general linear modelling . . . . 33

4.2.5 Winsorised Estimate using the Random Effects . . . . 34

4.3 Logistic Regression . . . . . . . . . . . . . . . . . 35

4.3 .1 Choice of Model . . . . . . . . . . . . . 40

4.4 Imputation . . . . . . . . . . . . . . . 41

4.4 .1 Introduction . . . . . . . . . . . . 41

4.4.2 Mechanisms of Missingness . . . . . . . . . . . . 42

4.4 .3 Related Studies . . . . . . . . . . . . . 45

4.4.4 Methods of Imputation . . . . . . . . . . . . 46

4.4.5 Choice of Variables and Imputation Method . . . . . 47

5 Exploratory Data Analysis $\quad 49$

5.1 Data Description . . . . . . . . . . . . . . 49

5.2 Demographic Characteristics . . . . . . . . . . . 53

5.2.1 Maternal and Birth Characteristics . . . . . . . . 58

5.2.2 Birth Interventions and mode of delivery . . . . . . . 61

5.3 Potential Predictors for PPH . . . . . . . . . . . . 63

5.3.1 District Health Boards . . . . . . . . . . . . . 63

5.3 .2 Demographics . . . . . . . . . . . . . 65

5.3.3 Maternal Birth Characteristics . . . . . . . . . 66

5.3.4 Mode of Delivery and birth interventions . . . . . . . 68

6 Unadjusted Comparison $\quad 71$

6.1 DHB Comparisons . . . . . . . . . . . . . . 71

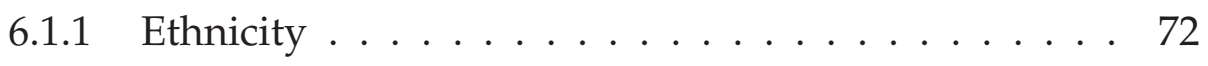

6.1 .2 Mother's Age . . . . . . . . . . . . . . 74

6.1 .3 Gender .................... 75

6.1 .4 Parity .................... 76

6.1 .5 Deprivation Index $\ldots \ldots . \ldots . \ldots 76$

6.1 .6 Summary . . . . . . . . . . . . . 77

6.2 Unadjusted Funnel Plot . . . . . . . . . . . . . . . 78 
6.2.1 Unadjusted Funnel Plots Method . . . . . . . . . . . . 78

6.2.2 Unadjusted Funnel Plot Results . . . . . . . . . . . . . 79

7 Risk Adjusted Comparisons $\quad 81$

7.1 Imputation $\ldots \ldots \ldots . \ldots \ldots 1$

7.1.1 Investigation of Variables to be Imputed . . . . . . . . 81

7.1.2 Imputation Method . . . . . . . . . . . . . . . 87

7.1.3 Imputation Results . . . . . . . . . . . . . . 90

7.1 .4 Summary . . . . . . . . . . . . . . 92

7.2 Logistic Regression Results . . . . . . . . . . . . . . . . 94

7.3 Risk Adjusted Funnel Plots . . . . . . . . . . . . . . . . . 97

7.3.1 Risk Adjusted Funnel Plot Results . . . . . . . . . . . 99

7.4 Winsorised Funnel Plots . . . . . . . . . . . . . . . . . . 101

7.4.1 Winsorised Estimates with Random Effects . . . . . . 104

7.4.2 Winsorisation Funnel Plot Results . . . . . . . . . 106

7.4.3 Winsorisation Random Effects Funnel Plot Results . . 107

7.4.4 Winsorisation Results Excluding Auckland DHB . . . 108

7.4.5 Winsorisation with Random Effects Results Excluding Auckland DHB . . . . . . . . . . . . . . . . 110

8 Discussion 113

$\begin{array}{ll}\text { A Glossary } & 121\end{array}$

B Imputation Regression Results 123

B.1 Gestation . . . . . . . . . . . . . . . . . . . . . 123

B.2 Parity . . . . . . . . . . . . . . . . . . . 124

B.3 Birth Weight . . . . . . . . . . . . . . . . 125

C Model Selection Results 129

C.1 Regression results for Adjusted Plot . . . . . . . . . . . 133 


\section{List of Figures}

5.1 Map of NZ DHBs . . . . . . . . . . . . . . . . 50

5.2 Frequency of Mother's Age . . . . . . . . . . . . . . 53

5.3 Percentage of mothers by Deprivation Index . . . . . . . . 55

5.4 Percentage of Mothers Ethnic Group by Age . . . . . . . . . 56

5.5 Deprivation Index by Mothers Age . . . . . . . . . . . . 57

5.6 Deprivation Index by Mothers Ethnicity . . . . . . . . . . 57

5.7 Gestation . . . . . . . . . . . . . . . . . 59

5.8 New borns birth weight . . . . . . . . . . . . . . 59

5.9 Age by Parity for all Mothers . . . . . . . . . . . . . . 61

5.10 Deprivation Index by Parity . . . . . . . . . . . . . 62

5.11 Mother's Ethnicity for mothers who have had a PPH . . . . 66

$6.1 \mathrm{PPH}$ comparison across DHBs . . . . . . . . . . . . 72

6.2 DHB by Ethnicity . . . . . . . . . . . . . . . 73

6.3 DHB by Median Age . . . . . . . . . . . . . . . 75

6.4 DHB by Parity . . . . . . . . . . . . . . . . . 76

6.5 DHB by NZ Deprivation Index . . . . . . . . . . . 77

6.6 Unadjusted Funnel Plot . . . . . . . . . . . . . . . . . . 79

7.1 Age Distribution, for the whole dataset, data where gestation is missing, data where parity is missing and data where birth weight is missing (as defined below) . . . . . . . 82 
7.2 Distribution of Ethnic Group (top) and Deprivation Index distribution (bottom) for the whole dataset, where gestation is missing, where parity is missing and where birth weight is missing . . . . . . . . . . . . . . . . 84

7.3 Distribution of birth weight, gestation and parity, before and after imputation . . . . . . . . . . . . 93

7.4 Matrix of Regression Variables for selection . . . . . . . . . 96

7.5 Matrix of Regression Variables for that are chosen . . . . . . 97

7.6 Log Odds Ratio . . . . . . . . . . . . . . . . . . . . 98

7.7 Adjusted Rate . . . . . . . . . . . . . . . . . 100

7.8 Winsorisation Funnel Plot . . . . . . . . . . . . . . . 106

7.9 Winsorisation Random Effects Funnel Plot . . . . . . . . . . 108

7.10 Winsorisation Funnel Plot Excluding Auckland DHB . . . . 109

7.11 Winsorisation Random Effects excluding Auckland DHB . . 111 


\section{List of Tables}

1.1 ICD10-AM Codes and definitions. . . . . . . . . . . 6

3.1 Standard prioritisation of ethnicity. . . . . . . . . . . 20

3.2 The numbers of records removed in the data clean step. . . 23

3.3 Mapping of Ethnicities . . . . . . . . . . . . . . . . . 24

5.1 The Total Number of Births for each DHB. . . . . . . . . . . . 51

5.2 The NZ Population for each DHB . . . . . . . . . . . . . 52

5.3 The Total Number of Mothers Ethnicity . . . . . . . . . . . 54

5.4 Frequency of Parity . . . . . . . . . . . . . . . . . 60

5.5 Type of Births Procedures . . . . . . . . . . . . . . 63

5.6 The number of Mothers who have had a PPH by DHB . . . . 64

5.7 Mother's Age by PPH . . . . . . . . . . . . . . . . . 65

5.8 Parity by PPH . . . . . . . . . . . . . . . . . . 67

5.9 New Born's birth weight for mothers who had a PPH . . . . 67

5.10 PPH by Gestation . . . . . . . . . . . . . . . . . . 68

5.11 Type of Birth by Postpartum Haemorrhage . . . . . . . . . . 69

7.1 The number of percentage of missing parity by PPH. . . . . 84

7.2 Percentage of missing records for Gestation, Birth Weight and Parity for each DHB. . . . . . . . . . . . . 85

7.3 Summary of Birth Weight Imputation . . . . . . . . . . . 91

7.4 Summary of Gestation Imputation . . . . . . . . . . . . . . . . . . . . . . . . . . .

7.5 Summary of Parity Imputation . . . . . . . . . . . . 92 


\section{Chapter 1}

\section{Introduction}

\subsection{Background}

This thesis studies the rates of Postpartum Haemorrhage (PPH) in New Zealand district health boards (DHBs). Funnel plots will display and show divergence in PPH rates between each DHB. This thesis used data supplied from the Ministry of Health (MoH) National Minimum Dataset (NMDS) and Maternal and Newborn Information Systems (MNIS).

Apart from annual reports published by the Ministry of Health (Ministry of Health, 2004) there is very little research in New Zealand that looks at DHBs and their outcomes in a medical context. DHBs are mainly measured against each other for economic reasons using administrative data and not for the interest of health outcomes, such as mortality related events. This research will specifically look at the rate of PPH for each DHB.

This topic is unique in New Zealand because it will investigate a medical outcome (PPH). Most research in this area has previously been based on mortality or Myocardial infraction rates. Secondly this type of research is useful to inform hospitals, providers and their funders to identify areas that require further in-depth self monitoring and quality improvement for 
health care in maternity.

\section{Funnel Plots}

A funnel plot is a way of displaying information visually for institutional comparison. It helps to identify institutions that are divergent from the average and those that are not (Spiegelhalter, 2005a). The funnel plot can be from a complex dataset and allows a visual comparison of data derived from multiple health care units such as hospitals, doctors and surgeons et cetra (Gale et al., 2006).

A funnel plot is a form of Statistical Process Control (SPC) Chart (Dover and Schopflocher, 2011) and can be used to study comparative performance measures (Gale et al., 2006). An SPC chart is also known as a Shewart chart or process-behaviour chart and is used to determine whether the process being monitored is in control or not. If a process being monitored is not in control then some observations from the process will be divergent from the rest. These charts are also useful in identifying variation amongst institutions. If variation has been identified, adjustments need to be made to bring the process back into control (Spiegelhalter, 2005b).

In a funnel plot a 'target' rate and an observed indicator for each institution are plotted against a precision measure. Control limits that are based on this precision measure are then added and form a 'funnel' around the target. The funnel is wider where precision is low and narrower as precision increases. This adds a visual line to the plot so we can see where the observations lie in relation to each other and the control limits. This identifies outliers, those who are performing well and those who are not (Spiegelhalter, 2005a). The control limits are similar to confidence intervals. When the control limits are plotted they can easily help interpret and identify the outlying institutions. 
If an observation lies near the horizontal target line this indicates that the number of outcomes observed is similar to the expected outcome. If an observation is below the horizontal line then the outcome observed is lower than expected. If the observation is above the horizontal line then the observed outcome is greater than expected (Coory et al., 2007).

An important advantage of a funnel plot is that it displays variation among the units which can be adjusted for. If overdispersion around the target occurs there are many methods that can be applied to adjust for this. Previous research has used the funnel plot to look at hospital units or patients against a specific outcome (Coory et al., 2007; Spiegelhalter, 2005a; Richardson et al., 2009).

A funnel plot is a useful graphic aid to display and compare institutions against each other without ranking. It identifies the divergent units and the most extreme units as well as the units closest to the average (Spiegelhalter, 2005a).

\section{Overdispersion}

Overdispersion is the presence of greater variability than expected in the dataset (Berk and MacDonald, 2008). It cannot be accredited to just a few institutions or change. It can arise because there can be many factors that can contribute to the excess variability. If contributing factors are not accounted for then the majority of institutions can be incorrectly labeled as abnormal (Spiegelhalter, 2005b).

There are many methods for dealing with overdispersion such as using an adjustment method or a winsorisation random effects method (Spiegelhalter, 2005a). The risk adjustment method measures factors that are associated with the excessive variability and can bring the process in control. The random effects method adds a constant to the sampling variance 
of each unit (Spiegelhalter, 2005a). Other methods include clustering the units into more homogeneous groups so that the comparisons can be made by comparing like with like. This is similar to benchmarking and can be considered as a form of risk stratification within the cluster (Spiegelhalter, 2005b).

\subsection{Structure of this Thesis}

Chapter Two is a literature review divided into two sections which discuss international and NZ based research. The research specifically looks at studies in a medical context using funnel plots and the performance of institutions, with specific examples that relate to this research analysis.

Chapter Three describes the National Minimum Dataset (NMDS) and examines how the data is collected, the coding of ethnicity and the quality issues with the dataset.

Chapter Four describes the methods that will be used in the following chapters or sections including Exploratory Data Analysis (EDA), Funnel Plots, Logistic Regression and Imputation.

Chapter Five presents the Exploratory Data Analysis (EDA) where demographic, maternal, type of birth, birth interventions and mode of delivery are explored. These explanatory variables are explored to identify relationships with PPH.

Chapter Six presents the unadjusted comparison results. Firstly DHB comparisons are explored. The relationship between the explanatory variables and the DHBs are analysed to identify differences among DHBs. The unadjusted funnel plot method and results are then presented. 
Chapter Seven presents the choice of imputation method used and the results from imputation. The logistic regression results are presented which are used for the risk adjusted funnel plot. The risk adjusted, winsorised and winsorisation random effects funnel plots results are presented and examined.

Chapter Eight is a discussion on the findings of this research and a conclusion of the results.

\subsection{Research Objectives}

There are three key objectives in this body of research:

1. To determine if there is a difference among DHBs in their rates of $\mathrm{PPH}$.

2. To model the factors that are associated with PPH.

3. To determine if risk adjustment reduces divergence in PPH rate among DHBs.

\subsection{Postpartum Haemorrhage}

This section describes the term Postpartum Haemorrhage (PPH) and the associated factors and outlines previous research about PPH. Throughout the chapter Postpartum Haemorrhage will be referred to as PPH (Ministry of Health, 2004).

$\mathrm{PPH}$ is the excessive bleeding of a genital tract following the birth of a baby. A primary $\mathrm{PPH}$ in more precise terms it is where the mother has 
had a blood loss of more than $500 \mathrm{ml}$ from the genital tract within the first 24 hours following delivery (Ministry of Health, 2004). A secondary PPH is blood loss of $500 \mathrm{ml}$ or more after 24 hours of delivery. (Ministry of Health, 2004). For this work primary PPH is the main focus.

It is assumed, in this study, a mother who had a PPH had one of the following diagnosis ICD-10-AM (the 10th revision of the ICD10 Australian modification) codes in table 1.1.

Table 1.1: ICD10-AM Codes and definitions.

\begin{tabular}{|l|l|}
\hline ICD10-AM Code & Description \\
\hline 16556700 & $\begin{array}{l}\text { Other management of postpartum } \\
\text { haemorrhage }\end{array}$ \\
\hline O720 & $\begin{array}{l}\text { Immediate postpartum haemorrhage, } \\
\text { within the first 24 hours (following } \\
\text { delivery of placenta) }\end{array}$ \\
\hline O721 & $\begin{array}{l}\text { Delayed and secondary postpartum } \\
\text { haemorrhage (after the first 24 hours } \\
\text { following delivery) }\end{array}$ \\
\hline O722 & $\begin{array}{l}\text { Postpartum haemorrhage due to co- } \\
\text { agulation defects }\end{array}$ \\
\hline
\end{tabular}

Definitions of PPH can vary. The main definition is classified as blood loss from the genital tract exceeding 500mls within 24 hours of delivery of the baby (Harrison, 1998; Mousa, 2007). In practical terms this is very difficult to measure and quantify. Blood loss can often be underestimated because of catchment of blood, as swabs and clothes are often used to contain the blood. Combs et al. (1991) states that primary PPH with a loss greater than $500 \mathrm{ml}$ occurs in $1-5 \%$ of deliveries in high-income families.

$\mathrm{PPH}$ is an associated risk factor for pregnancy. Excessive blood loss following birth can cause a severe drop in blood pressure leading to shock and death if not treated (University of Virginia Health System, 2010). Ma- 
ternal death from postpartum haemorrhage is extremely rare. Mousa (2007) found that in the UK, 1/100,000 deliveries are at risk of maternal death. In developing countries the risk is greater at about 1/1000 (Mousa, 2007).

Taylor et al. (2005) found that PPH has a higher risk following a second pregnancy after a C-section and found that women who did not have a $\mathrm{C}$-section were at lower risk of PPH.

Risk factors associated with PPH are placental abruption, multiple pregnancy, pregnancy induced hypertension, having many previous births, infections, obesity, induced labour, the use of forceps or vacuum assisted delivery, episiotomy, previous $\mathrm{PPH}, \mathrm{C}$-section, pre-eclampsia, race/ethnicity and many others (Combs et al., 1991; University of Virginia Health System, 2010; Harrison, 1998; Mousa, 2007; McCormic et al., 2002; Taylor et al., 2005; Waitemata District Health Board, 2006; Jacobs, 2012). Many symptoms can occur during PPH such as uncontrolled bleeding, decreased blood pressure, increased heart rate, decrease in the red blood cell count and swelling and pain in the tissues of the vaginal and perineal wall (Jacobs, 2012; Taylor et al., 2005; McCormic et al., 2002).

The reported incidence of PPH can vary. The incidence of PPH in the UK varies from $4 \%$ to $11 \%$. National Womens Hospital reports a high incidence of PPH with $18 \%$ of all women suffering a primary PPH of $500 \mathrm{mls}$ to $1000 \mathrm{mls}$ and $4 \%$ suffering a primary blood loss of greater than $1000 \mathrm{mls}$ (National Womens Hospital, 1996). Knight et al. (2009) found in 2006 that in Australia 10\% of mothers giving birth had a PPH. In Scotland the rate was 4 per 1000 women, Canada 5\% and US 3\%. University of Virginia Health System (2010) suggests that $4 \%$ of women have a PPH and it is more likely to occur with a caesarean birth. This shows measurment of blood loss for PPH can differ. 
PPH can be treated and managed prior and after pregnancy. Management can consist of medication, procedures or therapies (University of Virginia Health System, 2010; Mousa, 2007). The main risks factors for PPH associated with this analysis are instrumental delivery, caesarean section, episiotomy, birth weight, parity and ethnicity. 


\section{Chapter 2}

\section{Literature Review}

\subsection{Literature Review on Funnel Plots}

There are a number of international research publications that have looked at many outcomes for health care providers using funnel plots. Many studies have used mortality outcomes related to heart conditions for example myocardial infraction. This section will review previous international research using funnel plots in a medical context.

A study based in Queensland, Australia, (Coory et al., 2007) involved patients admitted to hospitals with Acute Myocardial Infraction (AMI). This study compares specific hospitals using cross-sectional analysis and sequential motoring based on control charts. The main issue that needed addressing is how to present such data in a meaningful way that is easy to interpret. As most hospitals data are collected for administration and funding use, it makes it difficult to have such measures for quality of care.

Control charts are used to display and detect unusual performance of hospitals. A crude rate of AMI can be misinterpreted, risk adjustment is required to take into account factors that are related to AMI. This is so that hospitals that treat higher risk patients are not unfairly penalised. There 
are several methods for risk adjustment such as risk adjusted cumulative sum (CUSUM), risk adjusted expected minus observed, and Cumulative Risk-Adjusted Mortality (CRAM) plots. For this study the expected minus observed was used for a risk adjusted method.

With the use of league table type methods, a few outlying hospitals can be identified as either high or low outliers. This ignores the differing levels of precision across hospitals due to varying sample sizes. This method has many limitations and a more preferable way to display hospital outcomes would be the use of a funnel plot. This allows a hospital specific measure to be plotted against a precision measure with confidence limits that form the funnel around the average or target. From this graphical representation one can easily determine where each hospital sits in relation to each other.

A study based in the UK, Gale et al. (2006) used funnel plots to look at the performance of health care professionals using Myocardial infraction data. The purpose was to use funnel plots to demonstrate that data from the MINAP (Myocardial Infarction National Audit Project) can be used to allow more meaningful interpretations. This study investigated 230 hospitals, approximately 100,000 patients. Gale et al. (2006) found that the funnel plot is a useful aid to display institutional comparison. It was quickly identified how many hospitals fell outside the 95\% control limits and how many were inside the control limits. Gale et al. (2006) concluded that funnel plots can be applied to complex data from multiple health care institutions, as it avoids such rankings as pass or fail for a hospitals' performance.

Dover and Schopflocher (2011) used funnel plots in public health surveillance. Dover and Schopflocher (2011) investigated the MVA (Motor Vehicle Accidents) death rate in 2007 in Alberta, Canada. The aim was to inves- 
tigate how to display the analysis, so that it is in the best interest of planning, implementation and evaluation of the public health system. This will allow public health policies to be made after monitoring the health status of the population.

The first approach uses a simple model to construct the first funnel plot which uses a crude motor vehicle mortality rate. It was found that $23 \%$ of the sub regions fall outside the $95 \%$ confidence limits. As this first approach shows large overdispersion a second approach was taken where a risk adjusted rate is used to take into the account variables that may be associated with MVA, such as the use of the seat belts, drink driving and road type etc. The adjusted rate then becomes the product of the provincial crude rate and the ratio of observed to expected values from the relevant regression model. A step by step approach was used for the model where a small set of factors was added to the regression model and analysed. Pearson's goodness-of-fit statistics were used at each stage of the modelling process to assess whether the adjusted variables were significant. Once the regression model was finalised the risk adjustment funnel plot showed that the overdispersion had been taken into account.

Dover and Schopflocher (2011) concluded that funnel plots are a useful tool for displaying small area data in health surveillance and detecting anomalies. It is also important to consider relationships among other variables to deal with overdispersion. Using this information the public health decision makers can discuss the implications and use for routine monitoring.

Ohlssen et al. (2007) conducted a larger study using a modelling framework to identifying unusual performance in health care providers. Crosssectional data were used from health care providers to detect unusual performance. The purpose was to create a structure for comparing methods, 
focusing on the estimation and hypothesis testing approach.

This paper discusses two alternative approaches that correspond to different interpretations of the word 'unusual'. The two approaches used were an estimation approach and a hypothesis based approach. The estimation approach is based on a single complex model in which the 'extreme' cases are identified. The hypothesis testing approach attempts to find a simple model to describe the cases which are of interest and that are divergent.

There are two study examples that are used in this paper by Ohlssen et al. (2007). The first is a patient-specific risk score that looks at coronary artery bypass graft patients, where the measure is 30 day morality rates for patients for each surgeon. Ohlssen et al. (2007) found that one surgeon was an outlier but could be treated as an over performer rather than ander performer. The second study contains routine data that are used in central monitoring of health services. It was based on data from the health care commission and looks at provider performance against emergency readmissions to hospital with 28 days of discharge.

This study by Ohlssen et al. (2007) concludes that there are three possible approaches to cross-sectional studies of unusual performance. The first, estimation of provider effects, requires flexible modelling assumptions and that the provider effects can be assessed using ranking or a set of external targets. The second, the hypothesis testing approach of each provider's outcome with an estimated null model using a classical p-value with no explicative alternative. And thirdly, a fully Bayesian hypothesis testing approach with an explicit alternative and calculation of posterior probabilities of the providers. The recommended approach is to use the hypothesis testing approach with no specific alternative and the use of $\mathrm{p}$ values to quantify unusual performance. 
This suggests that there are some key points that need to be considered. First, deciding on the null model for acceptable variation between providers. Secondly how to remove the influential providers that are potentially not in the null distribution or down weigh them (ie winsorisation). Thirdly, how to adjust for multiple comparisons.

\subsection{New Zealand Research}

A New Zealand based study by Richardson et al. (2009) looks at the ethnic and social-economic inequalities in mortality rates across regions. Using a hierarchical Bayesian approach, it uses posterior mortality rates from the models to calculate standardised rate ratios with credible intervals.

There are three main objectives that this study explores. Firstly to examine the mortality differences across regions in NZ by looking at income and ethnical inequalities. Secondly, to demonstrate the use of hierarchical Bayesian methods compared against simple empirical methods. Lastly, to investigate across regions the mortality rate for such factors as ethnicity, income, age and sex and to see how they differ among regions. The data used is the 2001 Census combined with the 2001-2004 mortality data in the New Zealand Census Mortality Study (NZCMS).

(Richardson et al., 2009) uses a hierarchical Bayesian regression approach to model the mortality rates in the 2001-2004 cohort, Richardson et al. (2009) found evidence of varying mortality rates among ethnic groups across NZ regions. There was no evidence for divergence among income groups in mortality across regions. If routine data were used it would report similar variability in NZ European mortality rates across DHBs but much greater variability in Māori morality rates and in Māori:European rate ratios. 
Richardson et al. (2009) concluded this is an interesting paper for the health service. They concluded that there are differences among regions in mortality rates and perhaps this should be considered when funding specific regions or DHBs. It is recommended that hierarchical Bayesian methods play an important role in prediction capabilities and displaying regional variation. This application allows more definite conclusions than routine empirical methods when comparing small populations.

Another NZ based study which has been recently published by Graham et al. (2012) looks at the variation among hospital outcomes using hierarchical Bayesian modelling and propensity scores to assess hospital performance. The study goes into great detail about how to adjust for comparing hospital performance with propensity scores methods to control case-mix factors. With the use of a Bayesian model this will allow assessment of performance on multiple outcomes. The sample population used is that of NZ public hospitals for patients that have been treated for AMI, pneumonia or stroke and the 30 day post admission mortality rate.

There are two main concerns that arise with the data and are addressed. Firstly, how to manage random variation and secondly the influence of case-mix adjustment. These are both important aspects of measuring performance as an incorrect measure could classify hospitals as having 'good' performance when it is actually 'poor' performance.

(Graham et al., 2012) shows how a hierarchical Bayesian method can improve the precision of the target and can control for any variation that may occur. Propensity scores are used here to obtain population standardised risk rates for each hospital and then the standardised risk rates are then modelled using hierarchical Bayesian method. 
Although there are specific limitations of this study were identified Graham et al. (2012) found the Bayesian method and propensity scores for assessing hospital performance for treatment against AMI, pneumonia or stroke is a valuable and practical method to measure and compare the performance of each hospital. 


\section{Chapter 3}

\section{Data Description}

The Ministry of Health's New Zealand Health Information Services collects and holds two datasets that contain information on hospital births. These are the National Minimum Dataset (NMDS) and the Maternal and Newborn Information Systems (MNIS). Data for this study were extracted from these two datasets. This chapter describes the data collection procedure, coding and quality issues for these two datasets (Ministry of Health, 2012a).

\subsection{Data Collection Procedure}

There are two sources which the $\mathrm{MoH}$ have provided and are used in this study. The NMDS and the MNIS both are described in more detail.

The NMDS provides unit record data about hospital events, including births. This information is provided by both publicly funded and private hospitals (Ministry of Health, 2012a) and includes information about health conditions and procedures used.

All hospitals whether public or private, must follow and use the interna- 
tional standard classifications of diseases and health problems, or ICD10-AM, the 10th revision of the ICD10 Australian modification codes. All records must also have a valid National Health Index (NHI) number. This is to match the hospital admissions data from NMDS (Ministry of Health, 2012a). Records are then sent to New Zealand Health Information Service (NZHIS) for validation and checking and then are loaded into the NMDS. Publicly funded hospitals have 21 days after the month of discharge to lodge the event. For public hospitals the files are electronic and these are processed daily. For paper and private hospital electronic records NZHIS has a team that manually processes these.

MNIS is data collected by services provided by Lead Maternity Caregivers (LMCs). It contains information and data on antenatal, birth and postnatal maternal services.

There are two sources where information is obtained from: the HealthPAC (health payment, agreements and compliances) and NMDS Systems. HealthPAC (health payment, agreements and compliances) previously known as Health Benefits Limited, collects information from claim forms submitted by LMCs. The main purpose of this system is to collect information about payments of LMCs for their services to pregnant women, mothers and babies. The payment information identifies services used and care given to the recipient (the mother and or baby). This information is then submitted to HealthPAC via a claim form (Ministry of Health, 2012a).

These claim forms are then forwarded to $\mathrm{MoH}$ and then in turn, entered into the co-payments database operated by HealthPAC. LMC claims are mainly used to provide primary information about payments and caregivers to facilitate payment of the LMCs. Therefore clinical and demographic information is of secondary nature and these areas of interest and have less scrutiny about the quality of information given. This informa- 
tion is supplemented once extracted from hospital records form the NMDS (Ministry of Health, 2012a).

MNIS was established in 2001. The MNIS contains information from 1999 onwards. The coverage of this information has improved over the years from $68 \%$ in 1999, for all births, deaths and marriages to $83 \%$ in 2003 and $77 \%$ in 2004 . The MNIS and NMDS are linked together through the women's NHI number which is matched to hospital admissions data from NMDS (Ministry of Health, 2012a).

\subsection{Coding of Ethnicity}

Ethnicity is the ethnic group or groups that people identify with or feel they belong to. Ethnicity is a measure of cultural affiliation, as opposed to race, ancestry, nationality or citizenship. Ethnicity is self perceived and people can belong to more than one ethnic group.

A person's ethnicity can change over time. $\mathrm{MoH}$ can record up to three ethnic groups for a person. Ethnic group is defined by $\mathrm{MoH}$ as "a group of people who have culture, language, history of traditions in common. Ethnicity is not the same as race, ancestry, or country of birth." (Ministry of Health, 2012b)

Collecting ethnicity can be problematic as providers or health care users are unwilling to divulge the information. Some can be unwilling to label themselves and some can be confused about the difference between ethnicity, nationallity, race or citizenship. $\mathrm{MoH}$ collects ethnicity data in the same way the NZ census ethnicity data are collected (Ministry of Health, 2012b; Statistics New Zealand, 2012). This is essential because hospitalisation rates are calculated by comparing the two datasets to determine pro- 
portions of the population hospitalised. $\mathrm{MoH}$ uses prioritised ethnicity according to Statistics NZ (Statistics New Zealand, 2012) by using specific algorithms that prioritised ethnic codes. Prioritised ethnicity is a hierarchical classification of ethnic group. For simpler analysis multiple records of ethnic groups per individual are prioritised using a standard by the NZHIS see table 3.1

Table 3.1: Standard prioritisation of ethnicity.

\begin{tabular}{|l|c|}
\hline Ethnicity & Prioritisation order \\
\hline Māori & 1 \\
Pacific peoples & 2 \\
South East Asian & 3 \\
Indian & 4 \\
Chinese & 5 \\
Other Asian & 6 \\
Other & 7 \\
Other European & 8 \\
European & 9 \\
Not Reported & 10 \\
\hline
\end{tabular}

\subsection{Data Quality Issues}

There are some known quality issues with the data which are described below. This information has been sourced from Ministry of Health (2004) and with personal communication from Information Analysts at $\mathrm{MoH}$. The quality issues are:

1. Incorrect reporting of parity as very little data quality work was done on this in the HealthPAC source system. This led to limited completeness and quality of parity data.

2. Incorrect reporting of Estimated delivery date (EDD). EDD was accepted if in an incorrect date format. 
3. The pregnancy identifier EDD field sometimes contained the provider ID instead of EDD.

Projects are underway to improve the quality and completeness of maternal and infant data in the MNIS National collection.

\subsection{Study Dataset}

The data used, has separate datasets for mothers and babies. These were then linked using the mother's NHI number and delivery date. The dates used to extract data are the babies' date of birth from 1/1/2007 to 31/12/2007. Several ICD-10-AM codes are used to identify where a mother has given birth. These include outcome of delivery, delivery code or a delivery procedure code. To identify birth events there will be one record per baby using the diagnosis of ICD-10-AM of live born infants.

\subsection{Data Cleaning}

In this section we describe the data cleaning that is required to prepare the data for the EDA and analysis presented in the next few chapters. As the dataset contained birth information from years 2004-2008 and there were separate tables for mothers and babies. Data processing and cleaning was necessary prior to the analysis.

1. Select 2007 year.

2. Selected matched mothers and babies only, otherwise not all variables are available for analysis (removed where match index is equal to $\mathrm{B}$ or $\mathrm{M}$ ) 
3. Remove cases where any DHB birth is overseas (as we are not interested in comparing overseas hospitals, only DHBs in NZ).

4. Removed cases where birth location of the hospital is not private or public (coded as 1 or 2).

5. Removed cases where mother's plurality is a not recorded (ie missing value).

6. Remove mothers where plurality is 2 or 3 , as this is recorded where the mother gave birth to twins or triplets. To restrict to single births.

7. Removed records where the deprivation index cannot be identified, this is where DHB of residence is overseas or not identified.

8. Remove multiple mothers that are not identified in the above steps. Removed mothers where mother's ID and babies DoB (Date of Birth) are the same as this indicates twins.

9. Removed multiple mothers second births because a mother may have had two births in 2007. Here we kept only the first birth. However this may cause a small bias if PPH is more (or less) likely the more children a mother has.

Table 3.2 shows the number of records removed in the data clean for each step.

\subsection{Derived Variables}

\section{PPH}

PPH is recorded as an ICD-10-AM code as based on V4 (April 09) FINAL ICD-10-AM 6th Edition Clinical Code Table. The codes descriptions are other management of postpartum haemorrhage, third-stage haemorrhage, 
Table 3.2: The numbers of records removed in the data clean step.

\begin{tabular}{|c|c|c|}
\hline Total Number & Difference & Description \\
\hline 65,784 & & $\begin{array}{l}\text { In the } 2007 \text { Year, total number of } \\
\text { records }\end{array}$ \\
\hline 57,478 & 8,306 & $\begin{array}{l}\text { Selected matched mothers and babies } \\
\text { only, so removed where matched in- } \\
\text { dex is B or M }\end{array}$ \\
\hline 57,427 & 51 & $\begin{array}{l}\text { Removed where DHB birth is } 999 \text { or } \\
x x x \text {. Where DHB is overseas. }\end{array}$ \\
\hline 57,426 & 1 & $\begin{array}{l}\text { Removed cases where birth location is } \\
\text { not private or public }\end{array}$ \\
\hline 57,426 & 0 & $\begin{array}{l}\text { Removed where any cases where gen- } \\
\text { der of the baby is unknown }\end{array}$ \\
\hline 57,419 & 7 & $\begin{array}{l}\text { Removed cases where mothers plu- } \\
\text { rality is NA }\end{array}$ \\
\hline 55,907 & 1,512 & $\begin{array}{l}\text { Removed mothers where plurality is } \\
2 \text { or } 3 \text {, so if plurality was recorded } \\
\text { with a } 2 \text { or } 3 \text {, that is twins or triplets, } \\
\text { these records are removed from the } \\
\text { analysis }\end{array}$ \\
\hline 55,890 & 17 & $\begin{array}{l}\text { Remove where DHB residence is } 999 \\
\text { or xxx }\end{array}$ \\
\hline 55,840 & 50 & $\begin{array}{l}\text { Removed multiple mothers that were } \\
\text { not identified in the above steps. Re- } \\
\text { moved mothers where their ID and } \\
\text { babies DoB birth are the same this in- } \\
\text { dicators twins }\end{array}$ \\
\hline 55,811 & 29 & $\begin{array}{l}\text { Removed multiple mothers second } \\
\text { births. As a mother may have had } \\
\text { two births in 2007, here we kept only } \\
\text { the first birth. }\end{array}$ \\
\hline & 55,811 & Total \\
\hline
\end{tabular}

other immediate postpartum haemorrhage and delayed and secondary postpartum haemorrhage. The codes are 1656700, O720, O721, O722 respectively. If any four of these diagnosis codes was assigned to the mother then PPH was set to 1, if a mother did not have anyone of these diagnosis codes then PPH was set to 0 for no PPH. 


\section{Mother's Age}

The age of the mother when giving birth. For the EDA this was grouped into the following age bands: 19 and Under, 20-24, 25-29, 30-34, 35-39 and $40+$. For the risk adjusted comparison age bands where not used and the mothers age was used.

\section{Ethnicity}

Ethnicity is recorded as 26 different ethnic groups. In this research the ethnicity groups were classed as one of the five groups: European, Māori, Pacific, Asian, Other or Non Response. Table 3.3 shows the grouping of ethnicity used for this research.

Table 3.3: Mapping of Ethnicities

\begin{tabular}{|c|c|c|}
\hline European & Māori & Pacific \\
\hline $\begin{array}{l}\text { European not further de- } \\
\text { fined } \\
\text { New Zealand European } \\
\text { Pakeha } \\
\text { Other European }\end{array}$ & Māori & $\begin{array}{l}\text { Pacific Peoples not further } \\
\text { defined } \\
\text { Samoan } \\
\text { Cook Island Māori } \\
\text { Tongan } \\
\text { Niuean } \\
\text { Tokelauan } \\
\text { Fijian } \\
\text { Other Pacific Peoples }\end{array}$ \\
\hline Asian & Other & NR \\
\hline Asian not further defined & Middle Eastern & Don't know \\
\hline Southeast Asian & Latin American/Hispanic & Refused to answer \\
\hline Chinese & $\begin{array}{l}\text { African (or cultural group } \\
\text { of African origin) }\end{array}$ & Response unidentifiable \\
\hline Indian & Other (retired 01/07/2009) & Not stated \\
\hline Other Asian & Other ethnicity & \\
\hline
\end{tabular}

\section{Gestation}

Is recorded in weeks. For the EDA has been grouped into the following categories: 0-37 weeks pre-term, 38-42 weeks normal, 42+ weeks post term. 


\section{Parity}

Parity is the number of times a mother has previously given birth and this is recorded from 0 to 14 times. For analysis work parity has been grouped into two categories: 1 for mothers who have never given birth before and 0 for mothers who have given birth at least once before.

\section{Breech}

This indicates if the mother has had a breech birth. Five different types of breech have been recorded. These are, an assisted breech delivery, assisted breech delivery with forceps, a breech extraction, a breech extraction with forceps and a spontaneous breech delivery. As breech birth numbers are very low these have been combined into one category called breech.

\section{Birth weight}

Birth weight is recorded in grams and for the EDA has been grouped into the following categories: $\leq 2499 \mathrm{~g}, 2500-4499 \mathrm{~g}$ and $4500 \mathrm{~g}+$. 


\section{Chapter 4}

\section{Statistical Methods}

In this chapter the statistical methods that are used in this thesis are described. This includes a description of the data that are investigated in the Exploratory Data Analysis (EDA) and an explanation of the funnel plots that will be analysed and displayed. The funnel plots include the unadjusted, risk adjusted and the winsorised funnel plots. Logistic regression and imputation methods are then presented.

\subsection{Exploratory Data Analysis}

The Exploratory Data Analysis (EDA) presents a summary overview of DHBs and their populations. It explores demographic characteristics such as age, ethnicity, deprivation index and the relationships between these characteristics. Maternal and birth characteristics including gestation, newborn's birth weight and parity are examined. Birth interventions and mode of delivery are explored. Potentially important predictors of $\mathrm{PPH}$ are presented and the relationships among the variables are investigated. Lastly, comparisons among DHBs are presented and the differences between DHBs in terms of demographic, maternal and birth characteristics and $\mathrm{PPH}$ are investigated. The EDA is a purely exploratory analysis where results are presented in tables and graphs. 


\subsection{Funnel Plots}

This section introduces components, functions and advantages of the funnel plot in the context of health related studies. A funnel plot is useful for providing a graphical representation of outcomes or health indicators across different providers (Spiegelhalter, 2005a). It is a scatter plot of an outcome against a measure of precision. An institution can be defined as a health provider, hospital, surgeon etc. In particular the funnel plot draws attention to the important relationships between the outcomes and volume of cases and can easily identify the outliers. (Sterne and Egger, 2001; Spiegelhalter, 2005a)

In the funnel plot the observed indicator for each institution is plotted against a precision measure. A 'target' indicator value is determined and a line is drawn as this value. The target can be the average across all institutions or a pre-specified value. Control limits are calculated as functions of the precision measure and then added to the plot to form a funnel around the target. This aids the understanding of where the observations lie in relation to each other and the control limits and identifies outliers, those who are performing well and those who are not (Spiegelhalter, 2005a).

With the aid of confidence limits it makes it easy to identify the outlying units. DHBs are used in this analysis however any unit of analysis could be used. Other analysis in the health sector have used hospitals, surgical teams, General Practitioners, or individual surgeons (Coory et al., 2007; Gale et al., 2006).

An advantage of funnel plots is that there are no false rankings of institutions compared to league tables where there must be rank order (Spiegel- 
halter, 2005a). For example in a league table one hospital must be at the top and one must be at the bottom. Another important aspect of a funnel plot is that it can allow for additional variation. If a funnel plot is over-dispersed then adjustments for this can be included, such as using winsorisation or adjustment for additional variables that are important to predict the outcome (Spiegelhalter, 2005a).

The funnel plot for proportions has four components

1. An indicator $Y$. In this study $Y$ is the proportion of births in which PPH occurs.

2. A target $\theta_{0}$ for $Y$ which denotes the expectation, so that $\mathbb{E}\left(Y \mid \theta_{0}\right)=\theta_{0}$ for the providers considered 'in control'.

3. A precision parameter $\rho$, which determines accuracy of the indicator. So for a given DHB that is in control and the target being achieved is the null distribution of $Y$ is taken as $p\left(y \mid \theta_{0}, \rho\right)$, where $\rho$ is proportional to the inverse of the variance of $Y$ where $n$ is the sample size. Since $Y$ is a proportion we have $\mathrm{E}\left(Y \mid \theta_{0}\right)=\theta_{0}$ and $\operatorname{Var}\left(Y \mid \theta_{0}\right)=\theta_{0}\left(1-\theta_{0}\right) / n$

Given that the first two moments of rate $p$ are

$$
\begin{aligned}
E\left[Y \mid \theta_{0}\right] & =\theta_{0} \\
\operatorname{Var}\left(Y \mid \theta_{0}\right) & =\theta_{0}\left(1-\theta_{0}\right) / n
\end{aligned}
$$


we can parameterise these using a location $\theta_{0}$ and precision $\rho$ where

$$
\begin{aligned}
\rho & =\frac{g\left(\theta_{0}\right)}{\operatorname{Var}\left(Y \mid \theta_{0}\right)} \\
& =\frac{g\left(\theta_{0}\right)}{\theta_{0}\left(1-\theta_{0}\right) / n}
\end{aligned}
$$

for some arbitrary function $g($.) The precision parameter can be made independent of $\theta_{0}$ by setting $g\left(\theta_{0}\right)=\theta_{0}\left(1-\theta_{0}\right)$, in which case $\rho=n$.

4. Control Limits $y_{p}\left(\theta_{0}, \rho\right)$ for a P-value $p$, where the chance of exceeding these limits for an in control unit is $p$. The control limits can then be calculated as

$$
Y_{p}\left(\theta_{0}, \rho\right)=\theta_{0} \pm Z_{p} \sqrt{\operatorname{Var}\left(Y \mid \theta_{0}\right)}=\theta_{0} \pm Z_{p} \sqrt{\frac{\theta_{0}\left(1-\theta_{0}\right)}{n}}
$$

Given a series of $I$ observations, $Y_{i}$ is associated with the precision parameter $\rho_{i}$. A funnel plot then can be constructed as a plot of $Y_{i}$ against $\rho_{i}$, with $\theta_{0}$ as the target which can be shown as a horizontal line and the control limits are superimposed on the plot as a function of the parameter $\rho$.

The above section describes the general form of the funnel plot. This now can be applied to the NMDS where the rate of PPH will be plotted against each DHB.

\subsubsection{Unadjusted Funnel Plot}

An unadjusted funnel plot uses the unadjusted rate, $Y_{i}$ and plots this against the population size $\rho_{i}=n_{i}$. The unadjusted plot is the first step to investigating and displaying the raw rates of $\mathrm{PPH}$ for each $\mathrm{DHB}$. This approach does not take into account that each DHB is made up of different popula- 
tion mixes which would need to be adjusted for.

\subsubsection{Risk Adjusted Funnel Plot}

Funnel plots are not limited to a single rate but can be used for more complex models to estimate the rate of interest. An risk adjusted funnel plot takes into account factors that may have influenced the outcome of interest. The risk adjustment is carried out using a judgment based modelling procedure where the choice of covariates are based on variation in patient mix that occurs between units (DHBs). This is to allow a for a fair comparison between DHBs to occur.

The target $\theta_{0}$ is still the national rate of PPH this is displayed as the centreline. A logistic regression model is used for the risk adjustment to calculate the expected number of events. The logistic model is used to derive a probability of an event occurring (say PPH) for individuals with a particular set of covariates. The predicted values for each $\mathrm{DHB}$ are summed over the set of individuals that have given birth in each DHB to give the expected number of events. This can be contrasted with the observed number of events $\mathrm{O}$ to give a standardised event ratio where this can be calculated by:

$\mathrm{E}=\sum_{i}^{n} \hat{p}_{i}$, where $\hat{p}_{i}$ is the predicted probability of the PPH individual $i$, $\mathrm{O}=\sum_{i}^{n} x_{i}$, where $x_{i}=1$ if PPH occurs and $x_{i}=0$ otherwise.

We then calculate the standardised ratio as $O / E \times N$, where $N$ is the national rate.

This is the risk adjusted rate which can then be plotted against each DHBs population. The control limits use the same formula as the unadjusted funnel plot, now with the adjusted rate (Spiegelhalter, 2005a). For more details on this approach and logistic regression, this is explained in more 
detail in section 4.3 .

\subsubsection{Winsorised Funnel Plots}

The previous two funnel plots methods unadjusted and risk adjusted are based on the assumption that the null target distribution, $f\left(y \mid \theta_{0}, \rho\right)$, fully expresses the variability of the in control units. However this may not be the case as the data could actually be 'overdispersed'. Overdispersion is the presence of greater variability than is expected in the dataset (Berk and MacDonald, 2008).

As the data becomes overdispersed it becomes increasingly necessary to take this overdispersion into consideration. If the above assumption is violated then this would suggest that the data is overdispersed (around the target). This may occur because of a larger number of unmeasured covariates that have not been accounted for in the risk adjusted method. The winsorisation approach attempts to adjust for this overdispersion (Spiegelhalter, 2005a)

There are two approaches to winsorisation

\section{A standard GLM approach}

2. Winsorisation using a random effects model

The GLM approach adds a factor $\phi$ that inflates the null variance and limits the extreme values and draws them into the mean. The GLM approach reduces the effect of large outliers (Spiegelhalter, 2005b). The random effects model adds a constant term to the sampling variance of each DHB unit. These methods are described in more detail in the next sections. 


\subsubsection{Winsorisation using general linear modelling}

The standard GLM approach will add a factor $\phi$, that inflates the null variance by a certain percentage factor $k$. This changes the extreme $Z-$ score values bringing them closer to the mean and the target. Which in turn reduces the treatment effect.

The precision parameter $\rho$, is calculated by

$$
\rho=g\left(\theta_{0}\right) / \mathbb{V}_{0}\left(Y \mid \theta_{0}, \rho\right)
$$

where the subscript on $\mathbb{V}_{0}$ has now been introduced to indicate this situation with no allowance for over-dispersion.

Here a multiplicative approach is used. This introduces a over-dispersion factor of $\phi$ that inflates the null variance, so that

$$
\mathbb{V}\left(Y \mid \theta_{0}, \rho, \phi\right)=\phi \mathbb{V}_{0}\left(Y \mid \theta_{0}, \rho\right)=\frac{\phi g\left(\theta_{0}\right)}{\rho}
$$

Note the previous equation used $g\left(\theta_{0}\right) \rho=\theta_{0}\left(1-\theta_{0}\right) / n$ to specify the variance. If all sample units $I$ are assumed to be in control then $\phi$ can be estimated by:

$$
\hat{\phi}=\frac{1}{I} \sum_{i} \frac{\left(y_{i}-\theta_{0}\right)^{2} \rho_{i}}{g\left(\theta_{0}\right)}=\frac{1}{I} \sum_{i} z_{i}^{2}
$$

where $z_{i}$ is the standardized Pearson residual given by

$$
Z_{i}=\frac{Y_{i}-\theta_{0}}{\sqrt{V_{0}\left(Y_{i} \mid \theta_{0}, \rho_{i}\right)}}
$$

The current control limits can then be inflated by a factor $\sqrt{\hat{\phi}}$ around $\theta_{0}$ based on the previous control limits. Over-dispersed control limits can be calculated as in equation 4.1 which also include the inflated factor $\hat{\phi}$ (Spiegelhalter, 2005a). 


$$
y_{p}\left(\theta_{0}, \rho\right)=\theta_{0} \pm z_{p} \sqrt{\hat{\phi} g\left(\theta_{0}\right) / \rho}
$$

\subsubsection{Winsorised Estimate using the Random Effects}

The second approach for adjusting for overdispersion is using the winsorised estimate as calculated above and adding a random effects term $\tau$. This is a additive variance model, so for each DHB the variance is increased by the same amount.

The random effects model assumes that $Y_{i}$ has an expected value of $E\left(Y_{i}\right)=$ $\theta_{i}$ and a variance of $\mathbb{V}\left(Y_{i}\right)=\sigma_{i}^{2}$. For 'on target' DHBs it is assumed that $\theta_{i}$ is drawn from distribution and $i$ is for each DHB, distributed with a mean $\theta_{0}$ and standard deviation $\tau$. A standard 'methods of moments' estimator can be used to calculate $\tau$ (Spiegelhalter, 2005a). The null hypothesis is now represented by a distribution rather than a single point.

In general

$$
E\left(Y_{i}\right)=\theta_{i}
$$

then

$$
\operatorname{Var}\left(Y_{i}\right)=\frac{\theta_{i}\left(1-\theta_{i}\right)}{n_{i}}
$$

where $n_{i}$ is the sample size from each $Y_{i}$ that is observed.

To adjust for overdispersion using a random-effects approach it is assumed that

$$
\theta_{i} \sim G\left[\theta_{o}, \tau^{2}\right]
$$

where $G\left[\theta_{o}, \tau^{2}\right]$ is a distribution with a mean $\theta_{o}$ and variance $\tau^{2}$.

We therefore estimate $\theta_{i}$ by $p_{i}$ the observed rate for each DHB

in this case

$$
\operatorname{Var}\left[Y_{i}\right]=\theta_{i}\left(1-\theta_{i}\right) / n_{i} \sim p_{i}\left(1-p_{i}\right) / n_{i}=s_{i}^{2}
$$


The method of moments estimate can be calculated as:

$$
\hat{\tau}^{2}=\frac{I \hat{\phi}-(I-1)}{\sum_{i} w_{i}-\sum_{i} w_{i}^{2} / \sum_{i} w_{i}}
$$

where $w_{i}=1 / s_{i}^{2}$,

$s_{i}^{2}=$ is the variance for each DHB proportion $p_{i}\left(1-p_{i}\right) / n_{i}$ and $\hat{\phi}$ is the test for heterogeneity if $\hat{\phi}<(I-1) / I$ then $\hat{\tau}^{2}$ is to be set to 0 and complete homogeneity is assumed.

The funnel plot confidence limits are given by:

$$
\theta_{0} \pm Z_{p} \sqrt{V_{0}\left(Y \mid \theta_{0}, \rho\right)+\tau^{2}}
$$

\subsection{Logistic Regression}

A common method for regression applications is the analysis of binary data in the use of a Logistic Regression Model. Binary responses are a common outcome that are studied in medical research. For example the death of a patient due to disease, the presence of disease and treatment outcomes after surgery or occurrence of PPH. For this research it is of interest to look at a response variable $\mathrm{Y}$ and see how a set of predicator variables $\mathrm{X}$ are related to $\mathrm{Y}$. For example these predictors could be demographic characteristics, type of treatment and risk factors etc. The outcome variable, known as the response, can take the value of 0 or 1 where $Y=1$ denotes where the event has occurred (Harrell, 2001).

First let's look at a linear regression model that is denoted by:

$$
E(Y \mid X)=x^{T} \beta
$$

For binary outcomes we wish to model $P\left(Y_{i}=1 \mid X\right)$, where $i$ indexes an 
individual patient if not a hospital, there are many predictors let $X$ denote a vector of $\mathrm{X}^{\prime} \mathrm{s}\left(X_{1}, X_{2}, \ldots X_{k}\right)$. If a linear regression model is used to model binary outcomes the predictions of $P(Y=1 \mid X)$ may not be constrained between 0 and 1 . Hence a generalized linear model is preferred for the analysis of binary response (Harrell, 2001).

A generalized linear model is made up of a linear predicator

$$
\eta_{i}=\beta_{0}+\beta_{1} x_{1 i}+\ldots+\beta_{p} x_{p i}
$$

and two functions a link function and a variance function. The link function describes how the mean, $E\left(Y_{i}\right)=\mu_{i}$, depends on the linear predicator using $g\left(\mu_{i}\right)=\eta_{i}$. The variance function describes how the variance, $\operatorname{var}\left(Y_{i}\right)$ depends on the mean. $\operatorname{var}\left(Y_{i}\right)=\phi V\left(\mu_{i}\right)$, where $\phi$ is the dispersion parameter.

For binary data the distribution is

$$
Y_{i} \sim \operatorname{Binomial}\left(1, \mu_{i}\right)=\operatorname{Bernoulli}\left(\mu_{i}\right)
$$

Where $\mu_{i}=P\left(Y_{i}=1\right)$

Then

$$
E\left(Y_{i}\right)=\mu_{i}
$$

and

$$
\operatorname{var}\left(Y_{i}\right)=\mu_{i}\left(1-\mu_{i}\right)
$$

Therefore the variance function is given by

$$
V\left(\mu_{i}\right)=\mu_{i}\left(1-\mu_{i}\right)
$$

The link function $g\left(u_{i}\right)$ must map $\mu_{i}$ from $(0,1)$ to the range $(-\infty, \infty)$ where, 


$$
g\left(\mu_{i}\right)=\operatorname{logit}\left(\mu_{i}\right)=\log \left(\frac{\mu_{i}}{1-\mu_{i}}\right)
$$

The logistic regression model links the mean $\mu_{i}=P(Y=1 \mid X)$ to the linear predicator using

$$
\begin{aligned}
\operatorname{logit}\left(\mu_{i}\right) & =\log \frac{\mu_{i}}{1-\mu_{i}} \\
& =X_{i}^{T} \beta
\end{aligned}
$$

If the factors are additive then this model will assume that for every predictors $X_{j}$

$$
\begin{aligned}
\operatorname{logit}\left(\mu_{i}\right) & =\operatorname{logit}\left(P\left(Y_{i}=1 \mid X\right)\right)=\beta_{0}+\beta_{1} X_{i 1}+\ldots+\beta_{j} X_{i j}+\ldots+\beta_{k} X_{i k} \\
& =\beta_{j} X_{i j}+C
\end{aligned}
$$

where if all other factors are held constant, $\mathrm{C}$ is defined

$$
C=\beta_{0}+\beta_{1} X_{1}+\ldots+\beta_{j-1} X_{j-1}+\beta_{j+1} X_{j+1}+\ldots+\beta_{k} X_{k}
$$

The parameter $\beta_{j}$ can be interpreted as the change in log odds for a unit change in $X_{j}$, if $X_{j}$ represents a single factor and all other factors remain constant. This can also be written in the terms of odds ratio:

$$
\begin{aligned}
\frac{\operatorname{odds}\left(Y=1 \mid X_{1}, X_{2}, \ldots, X_{j}+1, \ldots, X_{k}\right)}{\operatorname{odds}\left(Y=1 \mid X_{1}, X_{2}, \ldots, X_{j}, \ldots, X_{k}\right)} & =\exp \left[\beta_{j} X_{j}+\beta_{j}-\beta_{j} X_{j}\right] \\
& =\exp \left(\beta_{j}\right)
\end{aligned}
$$

Therefore, a one unit increase in $X_{j}$ increases the odds that $Y=1$ by a 
factor of $\exp \left(\beta_{j}\right)$. To demonstrate only one predictor $X$ will be used that has a binary outcome of 0 or 1 . The model can be written as:

$$
\begin{aligned}
& \operatorname{logit}(Y=1 \mid X=0)=\beta_{0} \\
& \operatorname{logit}(Y=1 \mid X=1)=\beta_{0}+\beta_{1}
\end{aligned}
$$

$\beta_{0}$ is the log odds where $Y=1$ and $X=0$. The difference between these two equations is $\beta_{1}$. This can be seen as the difference when $X=0$ and when $X=1$. Therefore the regression parameters can be interpreted by:

$$
\begin{aligned}
& \beta_{0}=\operatorname{logit}\left(P^{0}\right)=\log \left(P^{0} /\left(1-P^{0}\right)\right) \\
& \beta_{1}=\operatorname{logit}\left(P^{1}\right)-\operatorname{logit}\left(P^{0}\right)
\end{aligned}
$$

where $P^{0}$ is $P(Y=1 \mid X=0)$ and $P^{1}$ is $P(Y=1 \mid X=1)$. If we add a continuous predictor $X$ the regression equation becomes

$$
\operatorname{logit}(Y=1 \mid X)=\beta_{0}+\beta_{1} X_{1}
$$

where this model assumes a straight line in the log odds and that for one unit increase in $\mathrm{X}$ will increase the odds by a factor of $\exp \left(\beta_{1}\right)$.

This can be simplified to an odds ratio for each estimate as

$$
\frac{\operatorname{odds}(X=1)}{\operatorname{odds}(X=0)}=\exp \left(\beta_{1}\right)
$$

where $X=0$ is the reference level and for large samples the odds ratio has the $95 \%$ confidence interval:

$$
\exp (\hat{\beta}) \pm 1.95 \sqrt{\operatorname{Var}(\hat{\beta})}
$$




\section{Goodness of fit}

The goodness of fit test describes how well the model fits the data. There are few measures to assess the goodness of fit for logistic regression models. Pearson and Deviance residuals are calculated for logistic regression models and these measures can be assessed. Residual plots using the Deviance and Pearson residuals are used to check influential outliers and systematic trends.

The deviance can be used for unweighted data and is used to compare the maximised values of the log likelihood function for the maximal and the model of interest. The deviance is defined as:

$$
D=2\left[\log L_{\max }-\log L\right]
$$

where Lmax is the likelihood for the saturated model and L is the likelihood for the model of interested. In both cases the likelihood is evaluated at the maximum likelihood estimates of the parameters.

In large samples the deviance follows an approximate chi-square distribution $D \sim \chi_{n-p}^{2}$ where $n$ is the number of observations and $p$ is the number of parameters. The Pearson residuals can be tested using chi-square test. This compares the observed and expected values, using a chi-square distribution $\chi_{n-p}^{2}$.

A Hosmer-Lemeshow test can also be used to assess the goodness of fit for a binary logistic model. It tests whether or not the observed event rates match the expected event rates and follows a chi-square distribution with $n-2 \mathrm{df}$. 


\subsubsection{Choice of Model}

As there are a large number of possible explanatory variables to apply to the risk adjustment funnel plot, choosing the 'most appropriate' model can be a tedious task (Harrell, 2001).

An advantage with logistic regression and any regression model, is you can apply three different methods to identify the most 'appropriate model'. These methods are forward, backward and stepwise elimination. Below is a explanation about each choice of model selection. (Harrell, 2001)

\section{Backwards Elimination:}

This method starts with a model containing all the explanatory variables and eliminates variables one by one, at each stage choosing the variables for exclusion as the one leading to the smallest decrease in the AIC value. The AIC value is used to judge when further exclusions would represent a significant deterioration in the model.

Forward Selection:

This method starts with a model containing none of the exploratory variables and then considers variables one by one for inclusion, at each step the variable added is one that results in the biggest increase in AIC. An AIC value is used to judge whether further additions would represent a significant improvement in the model.

Stepwise Regression:

This method is essentially a combination of forward selection and backward elimination. It starts with no variables in the model and variables are added using the forward selection method. With each addition of a variable, a backward elimination process is considered to assess where the variables entered earlier might now be removed because they no longer contribute significantly to the model. The AIC value is used to detect vari- 
ables to be kept or removed, a entry and exit value can be used.

The stepwise logistic regression procedure considers a number of possible multiple regression models and selects subsets of parameters to test for possible addition to the model or elimination from the model. This is the most preferable method as it has the ability to add and remove covariates. The stepwise method is used to select the best model. This model is then applied to the imputation method used.

\subsection{Imputation}

This section presents approaches and methods for imputation. In detail it describes imputation, missing data mechanisms, the choice of variables and the most suitable imputation approach.

\subsubsection{Introduction}

Imputation is the substitution of a particular value for a missing value. It uses a set of methods, rules or a set of conditions that compute a value to be imputed for a missing value (Gelman and Hill, 2007).

As potential predictors for the model can have large numbers of missing values it is an essential step to impute these values. Without substituting these missing values the results will become biased. It can also severely reduce power and produce biased estimates (Arnold and Kronmal, 2003). There are many imputation methods for dealing with missing data and a selected few will be described below.

Imputation is used to prevent data becoming misleading of the population and non-representative of real world data. It is useful to impute values to allow the data to become more representative and help deal with missing 
data. One variable may be more susceptible to missingness than any other variable. It is sensible to fill the gaps (missing values) with sensible values which results in the imputation being unbiased (Gelman and Hill, 2007).

In $\mathrm{R}$, a language and environment for statistical computing and graphics, missing values are denoted by NAs. If values are missing the regression automatically excludes them and therefore can limit the amount of information available for analysis. These factors that could be removed can be potentially good predictors of the data model and if missing, biased estimates and results will be produced.

It is best to use imputation when there are small item non-response or missing values for critical variables, so that we can be confident that the effect of imputation is minimal, and that the methods used are strongly informed by the data. It is best to first evaluate variables and to identify what type of missingness there is prior to imputing values.

\subsubsection{Mechanisms of Missingness}

As there are many different imputation methods first useful to know why the data is missing and learn the pattern of missingness. This can help determine the most appropriate imputation method. There are four general conceptions of missing data which are known as missing data mechanisms. These are missingness completely at random (MCAR), missing at random (MAR), missing not at random (MNAR) and missing that depends on the missing value itself (not ignorable)(Gelman and Hill, 2007).

\section{MCAR (Missing Completely at Random)}

MCAR missingness means that the units with missing data can be treated as a simple random sample of the total sample: and in particular that miss- 
ingness does not depend on either the covariates or the outcome variable of interest. (Scheffer, 2002; Rubin, 1976). 


\section{MAR (Missing at Random)}

Here the probability that an individual value will be missing is independent of $Y^{(m)}$ (but may depend on $Y^{(o)}$ ). If this assumption holds likelihood based inferences are valid. For example if gender, ethnicity and age are provided but gestation is missing at random, then the probability of a non-response to this variable depends on these others being fully recorded variables. Here it is acceptable to exclude the missing cases, as long as the regression controls for all variables that affect the probability of missingness (Little and Rubin, 1989; Scheffer, 2002).

\section{MNAR (Missing Not at Random)}

Missingness that depends on unobserved predictors. When the probability that an individual value will be missing depends on $Y^{(m)}$ (Arnold and Kronmal, 2003).

\section{Missingness that depends on the value itself}

When the probability of missingness depends on the potentially missing variable itself. So for example one DHB could be more likely to not respond to a question for some reason, this could be to do with training, quality etc. If the observations with these missing values are discarded it can lead to results that are not truly representative of the DHB. Complete cases only could be a solution. However this would discard cases that are not complete cases and can lead to the above problem of biased estimates (Gelman and Hill, 2007). Bias is the biggest problem associated with NMAR missingness, and it cannot be corrected for. It cannot be tested for, since we lack the ability to detect the correlation between the response indicator and the unobserved outcome. 


\subsubsection{Related Studies}

A study by Schenker et al. (2006) on NHIS (National Health Interview Survey) health and income data describes and evaluates the methods and approaches of multiple regression imputation. The data is from the 2005 NHIS from the National Health Centre for Health. It is based on the National Health and Income Survey which contains a large proportion of non response for total family income and personal earnings from employment. The study uses multiple imputation which is similar to the approach that will be used with the NMDS. The method used takes the form; let $X$ denote the fully observed variables and let $Y^{(1)}, Y^{(2)}, \ldots, Y^{(k)}$ denote the $k$ variables with missing values, ordered by the amount for missingness from least to most. The process then follows for $Y^{(1)}, Y^{(2)}, \ldots, Y^{(k)}$ in $c$ rounds. In the first instance the regression of $Y^{(1)}$ on $X$ is fitted to the cases with $Y^{(1)}$ and the missing values of $Y^{(1)}$ are randomly imputed from the fitted model. Then the regression of $Y^{(2)}$ on $X$ and $Y^{(1)}$ is fitted with the $Y^{(2)}$ observed and then the $Y^{(2)}$ values are imputed and so forth. It is a continuous loop until all missing values for the variables listed are imputed. The main findings of this study found was that multiple imputation usually results in lower estimated standard errors than data that does not have any imputation.

Another study by Arnold and Kronmal (2003), describes the experience of using cardiovascular health study of imputing missing data on a large number of variables that were collected at baseline. The data used in this study was designed to identify risk factors for cardiovascular disease in individuals aged 65 years and over. When participants were first examined (this is the baseline measure) an extensive clinical examination was conducted. Multiple imputation was applied to missing data where an iterative approach is used on these variables.

When it comes to the choice of predictors in the imputation model it is 
important to include a large number of predictors and is also necessary to use such variables that will be used in further analysis (Rubin, 1996; Meng, 1994; Schenker et al., 2006).

\subsubsection{Methods of Imputation}

There are many different methods for imputing values. It is important to choose the most sensible approach for the type of data that will be imputed. Some methods of imputation which potentially could be used for this data are described below.

\section{Mean Imputation}

Mean imputation imputes the mean value for all missing values. It takes the mean of observed values of the variable for complete data and imputes this value into all missing values. It gives a sensible overall mean, assuming the data is MAR. This approach can only be done for continuous outcome variables However it can distort the distribution. This approach also ignores the relationships among variables.

\section{Hot Deck Imputation - within classes}

This approach divides data into imputation classes then replaces each missing value with the data from a randomly chosen donor in the same class. This method assumes MAR (where $X=$ imputation class), it preserves distributions within the classes and it works well with moderately large classes $(30+)$. This method finds it difficult to deal with multivariate $(\mathbf{X}, \mathbf{Y})$ relationships.

\section{Hot Deck Imputation - Distance based}

Chooses values from the most similar donors available and is based on a multivariate distance. This method can choose the best match or randomly 
from $k$ best. As it is multivariate distance it is possible to limit the donor usage by including a penalty for heavy use of using values further away. This model is more robust as it allows for multivariate $(\mathbf{X}, \mathbf{Y})$ relationships, though it can be less efficient to run.

\section{Regression Imputation}

In this approach, missing values are estimated based on predicted values generated by a regression model. The regression model is based on known variables for the missing unit of observation and requires that all values used as predictors are complete cases for the variables used in the regression.

\section{Multiple Imputation}

Rubin (1987) describes multiple imputation as a technique used that replaces each missing value with two or more values representing a distribution of possibilities. In summary the method of multiple imputation involves firstly imputing data under an appropriate model of choice and repeating this step to ensure the data is filled in. Secondly, analyse each dataset and or variable separately to identify and obtain desired parameter estimates and standard errors. And lastly combine all results from above to form a full dataset (Fox, 2008; Arnold and Kronmal, 2003).

\subsubsection{Choice of Variables and Imputation Method}

As there are a variety of imputation methods to choose from how do we know which is the most appropriate to use?

If mean imputation were to be used it assumes that the data is MAR. However this could severely distort the distribution as there are several variables that require imputation that are continuous. It would not make 
much sense to impute via this method. If hot deck imputation was chosen, donors of similar characteristics are chosen which allows for multivariate relationships. This is a more preferable method. For the NMDS we may want to impute through matching. For example each unit of $Y$ that is missing, find a unit with the same values for a set of predictors, $X$ in the observed data and copies the $Y$ value. This would be a sensible approach as it would not distort the data. The best approach would be to use a multiple imputation regression approach.

The advantage of regression imputation approach is that you can use a separate set of regression models for each $Y$ and is easier to fit a more reasonable model. The disadvantage is that you have to be careful in ensuring that the separate regression models are consistent with each other so that all regression models should be fairly similar (Gelman and Hill, 2007).

There are several variables that have a large number of missing values that are important predictors of PPH. These are gestation, parity and birth weight. First these three variables need to be investigated and explored to determine the pattern of missingness. 


\section{Chapter 5}

\section{Exploratory Data Analysis}

In this section the population of interest is defined and then explored. The Exploratory Data Analysis (EDA) presents a summary overview of DHBs and their population demographic characteristics such as age, ethnicity, deprivation index and their relationships. Maternal and birth characteristics are then presented. These include gestation, newborns birth weight and parity. Birth interventions and model of delivery are explored and lastly the potential predictors of $\mathrm{PPH}$ are presented and relationships among variables are explored. This establishes our expectations about relationships among the variables of interest. EDA is a purely an exploratory analysis where table and graphs are presented displaying but not quantifying relationships among two or three variables at a time.

\subsection{Data Description}

In 2007 New Zealand was divided into 21 DHBs. See Figure 5.1 for a map of NZ DHBs.

In the 2007 data there were 56,681 distinct mothers who gave birth to 57,478 babies. For this analysis we are interested in single births therefore 


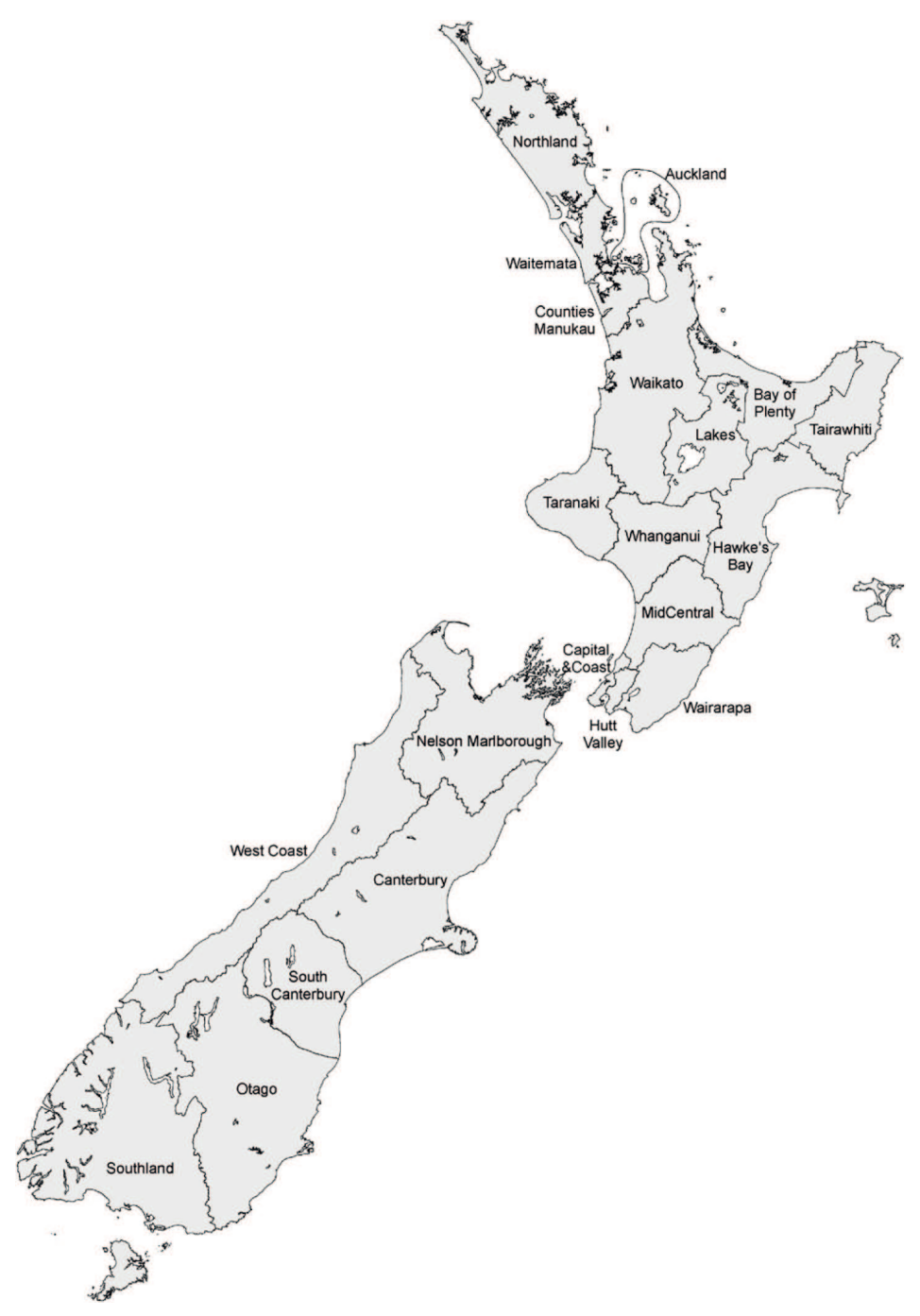

Figure 5.1: Map of NZ DHBs

mothers who gave birth to more than one baby were excluded. Mothers who gave birth overseas or who are overseas residents are also excluded from the analysis. See section 3.5 for a more detailed description of exclusions from the dataset. The analysis in this section will only look at singleton births. There are 55,811 mothers who gave birth to 55,811 babies. This is the total number of mothers that will used in the analysis. 
Table 5.1: The Total Number of Births for each DHB.

\begin{tabular}{|l|r|}
\hline District Health Board & Total Number of Births (\%) \\
\hline Counties Manukau & $7,986(14.13 \%)$ \\
Waitemata & $6,797(12.18 \%)$ \\
Auckland & $6,091(10.91 \%)$ \\
Canterbury & $6,039(10.82 \%)$ \\
Waikato & $4,757(8.52 \%)$ \\
Capital and Coast & $3,511(6.29 \%)$ \\
Bay of Plenty & $2,616(4.69 \%)$ \\
MidCentral & $2,004(3.59 \%)$ \\
Hawke's Bay & $1,986(3.56 \%)$ \\
Hutt & $1,918(3.44 \%)$ \\
Otago & $1,876(3.36 \%)$ \\
Northland & $1,850(3.31 \%)$ \\
Nelson Marlborough & $1,482(2.66 \%)$ \\
Taranaki & $1,430(2.56 \%)$ \\
Lakes & $1,380(2.47 \%)$ \\
Southland & $1,359(2.44 \%)$ \\
Whanganui & $783(1.40 \%)$ \\
Tairawhiti & $649(1.16 \%)$ \\
South Canterbury & $602(1.08 \%)$ \\
Wairarapa & $455(0.82 \%)$ \\
West Coast & $340(0.61 \%)$ \\
\hline Total & $55,811(100 \%)$ \\
\hline
\end{tabular}

Table 5.1 shows the number of births in each DHB in 2007 in rank order. Counties Manukau has the largest proportion of births at $14.13 \%$ and West Coast has the smallest proportion of births in NZ at $0.61 \%$.

It is not surprising that the first four DHBs listed in table 5.1 have the highest proportion of births, (Counties Manukau, Waitemata, Auckland and Canterbury, $14.13 \%, 12.18 \%, 10.91 \%$ and $10.82 \%$ respectively) as these are the largest populated areas in New Zealand (Statistics New Zealand, 2012). Table 5.2 shows Waitemata, Canterbury, Counties Manukau and Auckland have $10-12 \%$ of the population and therefore should be expected 
to have the highest number of births. Likewise that South Canterbury, Wairarapa and West Coast (1.08\%,0.82\% and $0.61 \%$ respectively) have the smallest number of births in New Zealand as these are small populated areas. This can be compared to table 5.2 where West Coast, Wairarapa and South Canterbury only have $1 \%$ of the NZ population each. From this table 5.1 also demonstrates that there is a large variation in numbers of births per DHB. Some DHBs have a very small number of births compared to other DHBs which report a very large number of births.

Table 5.2: The NZ Population for each DHB

\begin{tabular}{|l|r|}
\hline District Health Board & Population (\%) \\
\hline Waitemata & $481,611(11.96 \%)$ \\
Canterbury & $466,407(11.58) \%$ \\
Counties Manukau & $433,086(10.75) \%$ \\
Auckland & $404,619(10.05 \%)$ \\
Waikato & $339,192(8.42 \%)$ \\
Capital and Coast & $266,658(6.62 \%)$ \\
Bay of Plenty & $194,931(4.84) \%$ \\
Otago & $179,397(4.45 \%)$ \\
MidCentral & $158,841(3.94 \%)$ \\
Northland & $148,440(3.69 \%)$ \\
Hawkes Bay & $148,248(3.68 \%)$ \\
Hutt & $136,101(3.38 \%)$ \\
Nelson Marlborough & $130,062(3.23 \%)$ \\
Southland & $106,827(2.65 \%)$ \\
Taranaki & $104,277(2.59 \%)$ \\
Lakes & $98,319(2.44 \%)$ \\
Whanganui & $62,211(1.54 \%)$ \\
South Canterbury & $53,877(1.34 \%)$ \\
Tairawhiti & $44,463(1.10 \%)$ \\
Wairarapa & $38,613(0.96 \%)$ \\
West Coast & $31,326(0.78 \%)$ \\
\hline Total & $4,027,506(100 \%)$ \\
\hline
\end{tabular}




\subsection{Demographic Characteristics}

In this section the demographic characteristics of the 2007 NMDS investigates age, ethnicity, deprivation index, gender of the baby and their relationships are explored.

\section{Mother's Age}

Figure 5.2 shows the percentage of mothers by age. This distribution shows the age of mothers range from 13 to 51 years. Most mothers giving birth in 2007 are aged 32 and 33 years. The average age of the mother is 29 years. There are smaller proportions of mothers aged under 19 years and over 40 years.

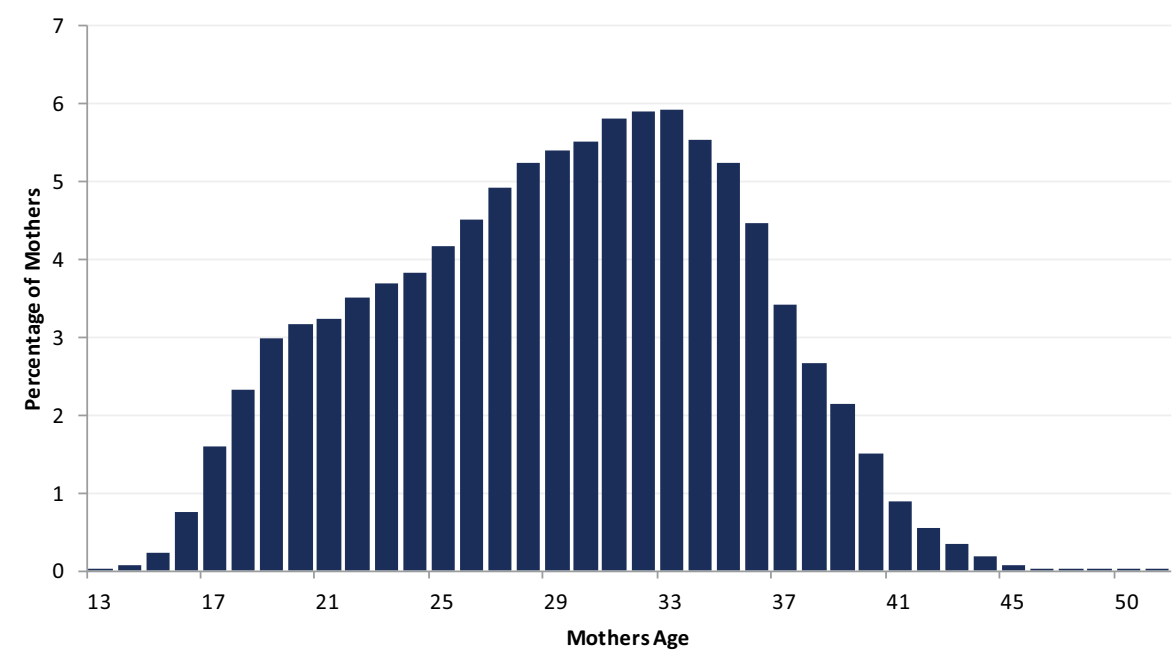

Figure 5.2: Frequency of Mother's Age

\section{Mother's Ethnicity}

The largest proportion of mothers are of European ethnicity (58\%), as shown in table 5.3 followed by Māori, (19\%), Pacific (10\%) and Asian (9\%) ethnicity. Other and Unknown are very small proportions (3\% and 1\% respectively) of mothers who gave birth in 2007. There are 398 missing ethnicity 
values.

Table 5.3: The Total Number of Mothers Ethnicity

\begin{tabular}{|c|c|c|c|c|c|c|}
\hline European & Māori & Pacific & Asian & Other & Unknown & Total \\
\hline $32,204(58 \%)$ & $10,804(19 \%)$ & $5,712(10 \%)$ & $5,276(9 \%)$ & $1,417(3 \%)$ & $398(1 \%)$ & 55,811 \\
\hline
\end{tabular}

\section{Deprivation Index}

Deprivation Index is an index of socioeconomic deprivation based on the 2006 census data, and calculated for each meshblock from Statistics NZ (Statistics New Zealand, 2012). The ordinal scale ranges from 1 to 10, where 1 represents the areas with the least deprived and 10 the most deprived areas. The deprivation index is derived from nine factors from the 2006 census and combined into a score for each meshblock, reflecting 10 different deprivation scores. A meshblock is defined as a geographical unit defined by Statistics New Zealand, for 2006 it contains a median of approximately 87 people (Statistics New Zealand, 2012).

As shown in Figure 5.3 a large proportion of mothers live in the most deprived areas. There are smaller proportions of mothers who live in the least deprived areas. $14.61 \%$ of mothers are situated in the deprived index of 9 , and $13.81 \%$ in the deprived index of 10 . At the least deprived indices of 1,2 and 3 . There are $7 \%$ of mothers in each of these areas. There are 37 missing values for this variable.

\section{Gender}

Of the 55,811 babies born $48 \%$ of births are female and $52 \%$ are male.

\section{Mother's Ethnicity by Mother's Age}

Figure 5.4 shows mother's ethnic group by age. European is the largest ethnic group for all age groups over 20 years. In the 19 years and under age group, $48 \%$ of mothers are Māori and 37\% are European. This age group 


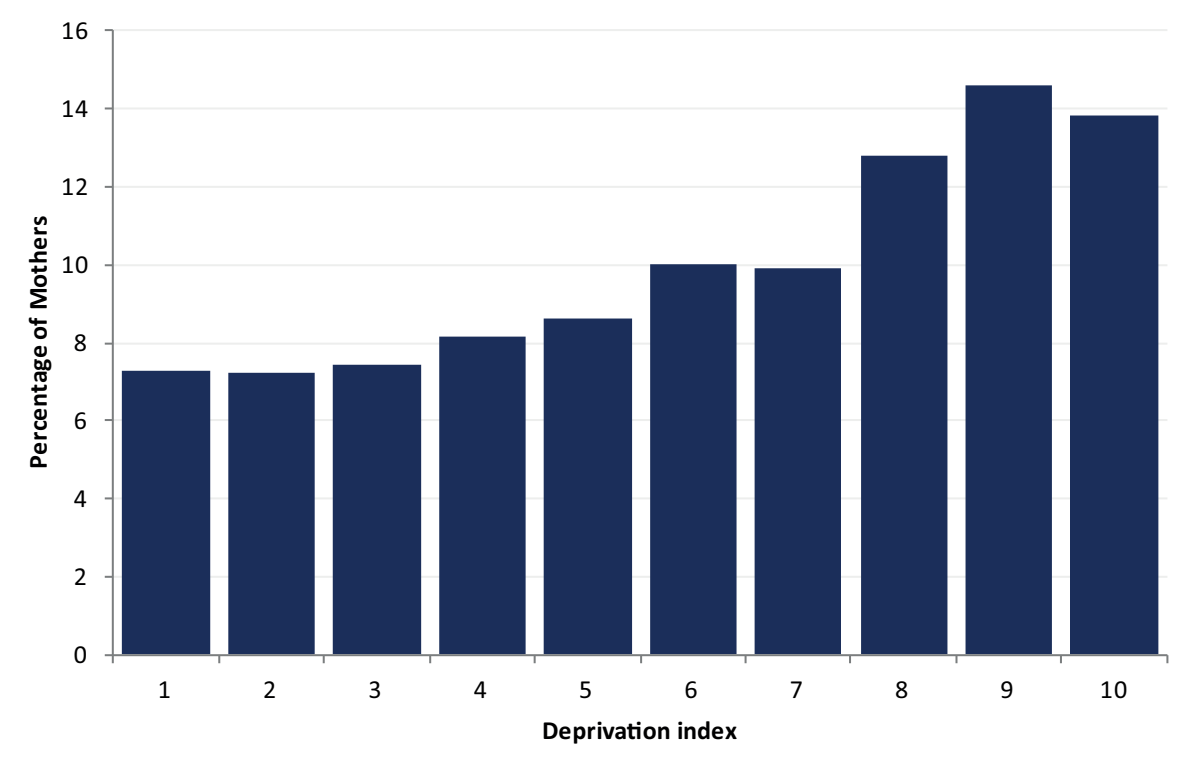

Figure 5.3: Percentage of mothers by Deprivation Index

has the largest proportion of Māori ethnicity compared to any other age group. Given that Māori mothers are 19\% of all births in 2007, and Māori are $15 \%$ of the NZ population they are over-represented in the 19 and Under and 20-24 age group (Statistics New Zealand, 2012). For the 20-24 age group there are 32\% Māori and $43 \%$ are European. For the Asian Ethnicity group the largest proportion of mothers are in the 25-29 age group at $12 \%$ and also in the 30-34 age group at $11 \%$. Pacific People have large proportions of mothers in the 20-24 age group at 15\% and at the 25-29 age group at $12 \%$. A chi-squared test shows that there is an association between age group and ethnicity $\left(\chi^{2}=5967, \mathrm{df}=20, \mathrm{p}\right.$-value $\left.<0.0001\right)$.

\section{Mother's age by Deprivation Index}

Large proportions of women aged 30-34 years are in the least deprived areas of 1-4, with approximately $35 \%$ in each index and small proportions of these mothers in the most deprived area 10 at $20 \%$. For the younger women in the 19 and under age group, $14 \%$ of mothers are based in the 


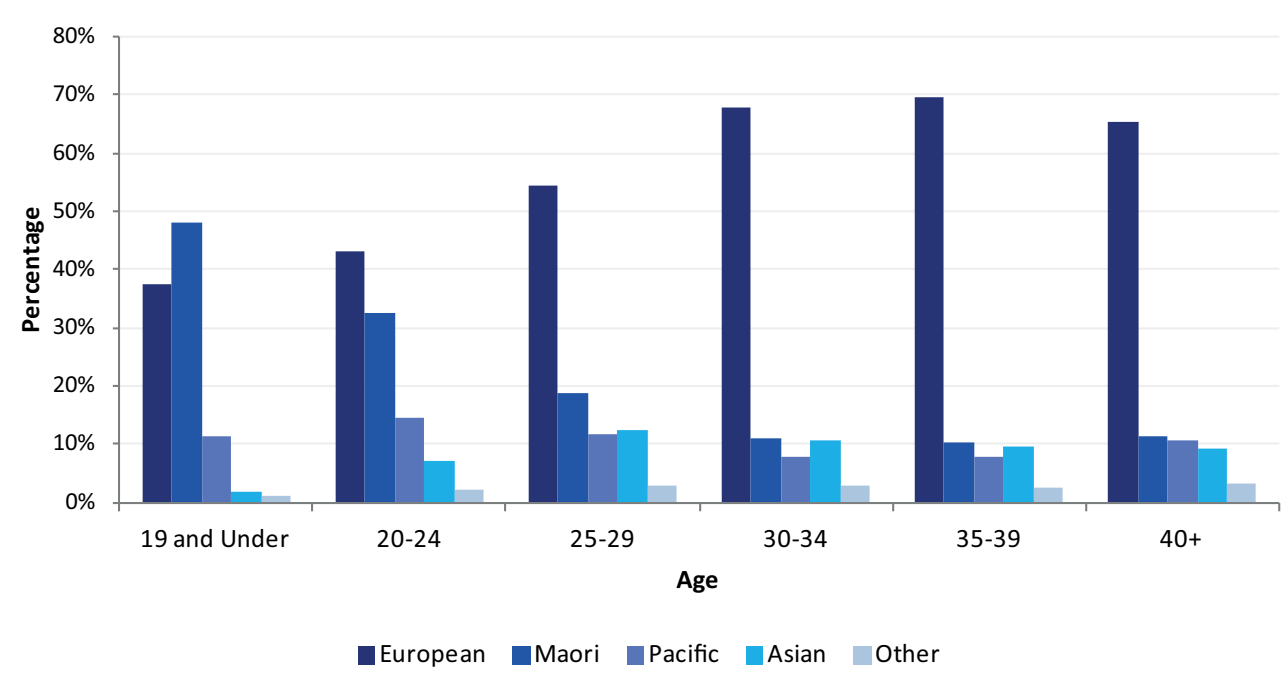

Figure 5.4: Percentage of Mothers Ethnic Group by Age

most deprived areas of 10 and $2.9 \%$ based in the least deprived area of 1 . Also mothers who are in the age group 20-24 years have large proportions of mothers in the most deprived areas, compared to the least deprived areas, $26 \%$ to $6 \%$. For the $25-29$ age group there are even proportions of mothers across each deprivation index, see figure 5.5. A Chi-square test shows that there is an association between deprivation index and mothers age $\left(\chi^{2}=3754, \mathrm{df}=45, \mathrm{p}\right.$-value $\left.<0.0001\right)$.

\section{Deprivation Index by Mothers Ethnic Group}

There is an association between mother's ethnic group and deprivation index $\left(\chi^{2}=11398, \mathrm{df}=36, \mathrm{p}\right.$-value $\left.<0.0001\right)$. There are large proportions of European ethnicity in deprivation index 1-8, and smaller proportions in deprived decilesS 9 and 10, see figure 5.6. In the deprivation index 10, there are large proportions of Māori, 40\%, Pacific 31\% and 20\% European. In the deprivation index of 9, 29\% are Māori, 44\% European and $13 \%$ Pacific. This shows these two deprivation areas are made up of different proportions of ethnic groups. The Asian ethnic group is a constant 


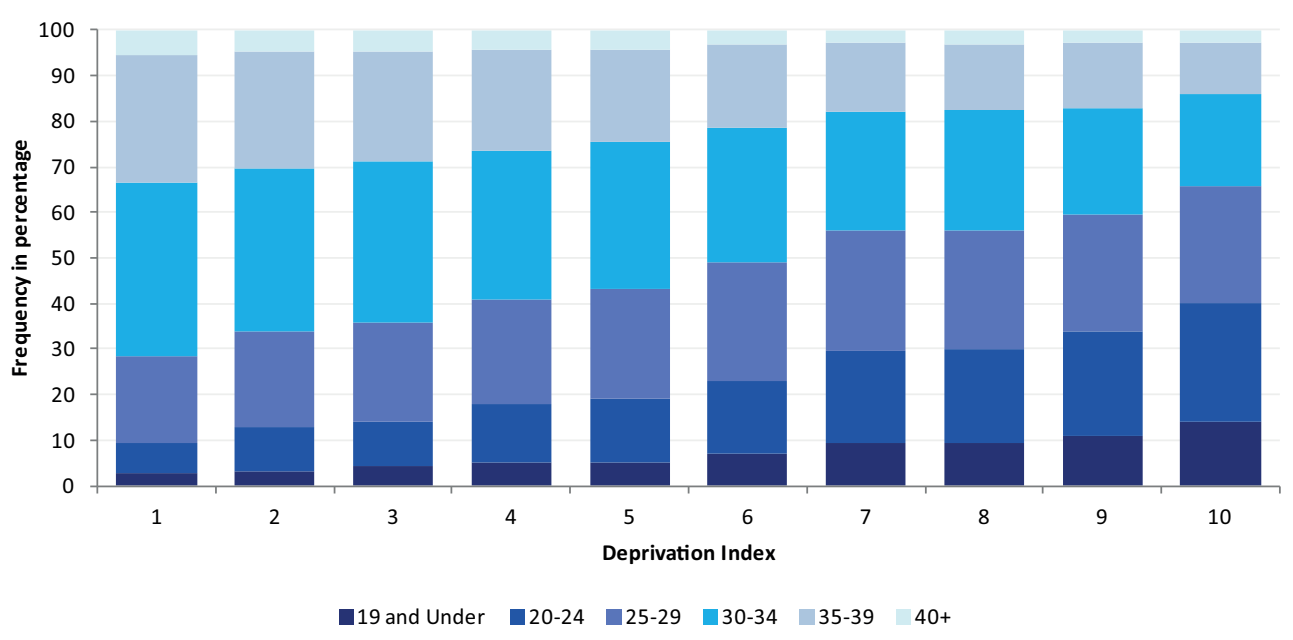

Figure 5.5: Deprivation Index by Mothers Age

proprotion across the indices, approximately $10 \%$ in each area. The most deprived area is the only exception here with only $6 \%$ being Asian in this group.

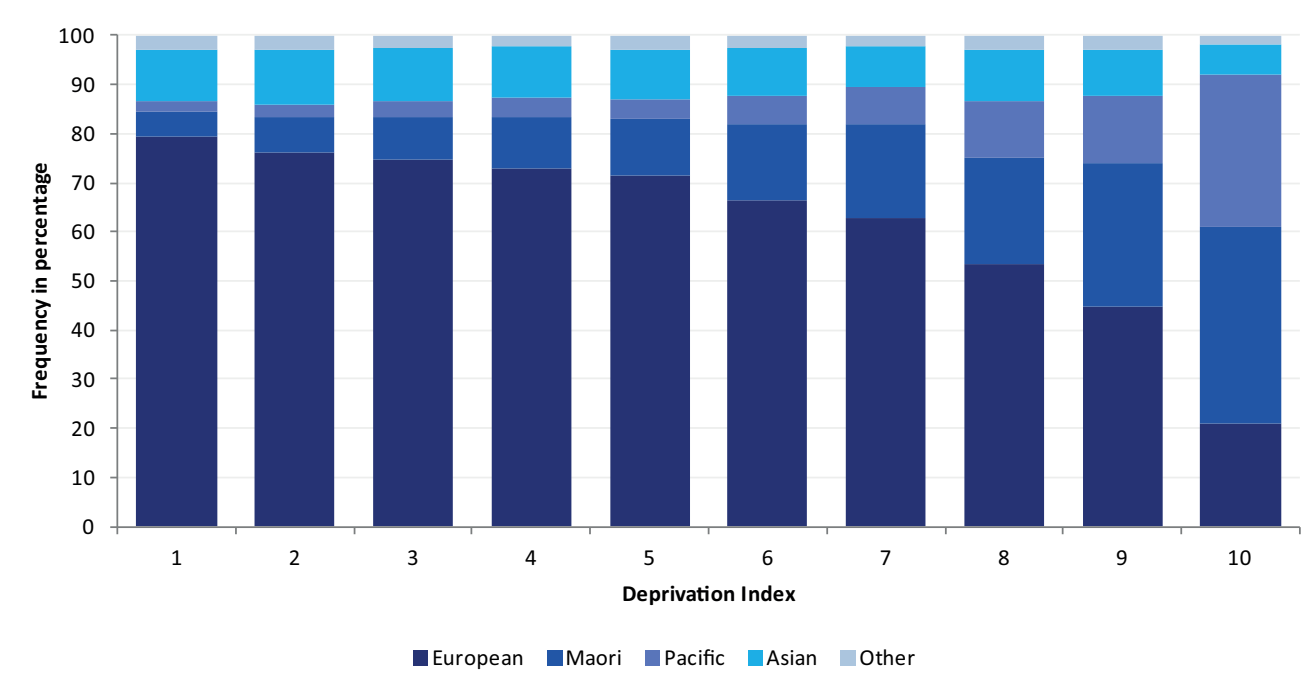

Figure 5.6: Deprivation Index by Mothers Ethnicity 


\subsubsection{Maternal and Birth Characteristics}

This section presents some key maternal and birth characteristics. These include gestation, birth weight, parity and PPH. These factors and their relationships are explored.

\section{Postpartum Haemorrhage}

There are 55,811 mothers who gave birth in NZ in 2007. Of these 5,249 (9.40\%) had a PPH and 50,562 (90.60\%) mothers did not have a PPH.

\section{Gestation}

Gestation is recorded in weeks and shows the length of pregnancy until a mother gives birth. A normal pregnancy can range from 37 to 42 weeks. If an infant is born before 37 weeks this is considered pre-term and infants born after 42 weeks are considered a post-term birth. We would expect to see in 2007, NMDS the majority of births occurring between 37 to 42 weeks (Ministry of Health, 2012b).

Figure 5.7 shows there are very small proportions of mothers who have given birth between 14-34 weeks. At 37 weeks this number increases dramatically to a large peak at around 40 weeks. This then drops just as steadily as it rose at 42 weeks. This variable has 1,009 (1.84\%) missing values.

\section{Birth weight}

The average full term baby weighs $3500 \mathrm{~g}$ (seven and a half pounds, (Ministry of Health, 2012a). Around 95\% of babies weigh between $2500 \mathrm{~g}$ and $4250 \mathrm{~g}$. If a baby weighs less than $2500 \mathrm{~g}$, this is considered a low birth weight. In the NMDS, birth weight ranges from $225 \mathrm{~g}$ to $7000 \mathrm{~g}$, with an average weight of $3466 \mathrm{~g}$. The data shows a left-skewed distribution of birth weight, with the highest proportion of birth weights at around $3600 \mathrm{~g}$ as seen in figure 5.8. There are 448 missing values for this variable. 


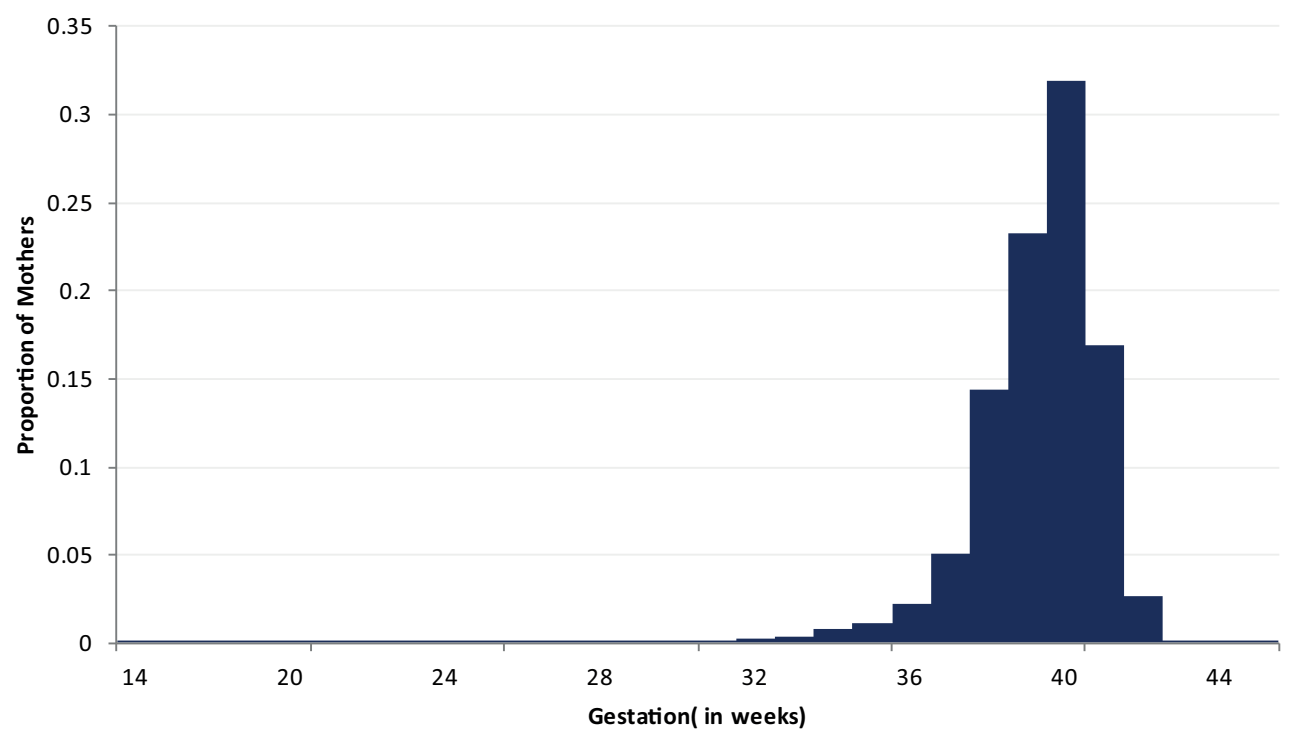

Figure 5.7: Gestation

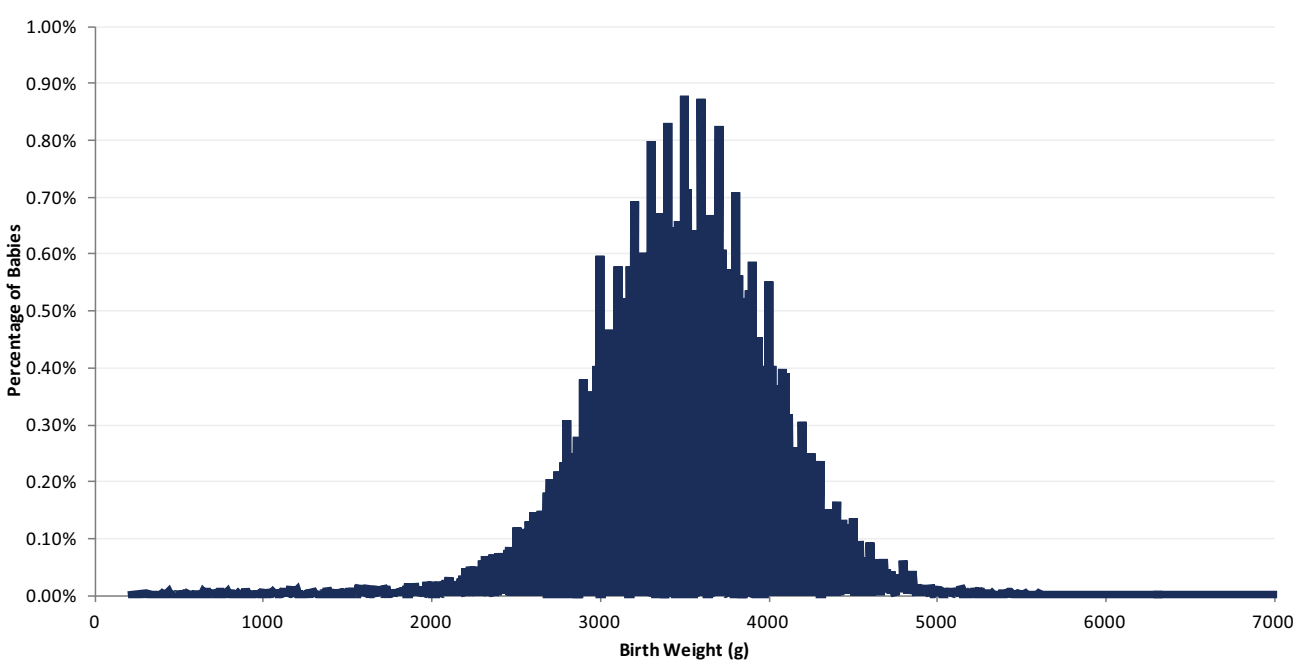

Figure 5.8: New borns birth weight

\section{Parity}

There are 19,014 (34.07\%) new mothers, who had never given birth before 
and 5,774 (10.35\%) mothers have previously given birth once before, the most extreme is a mother who has given birth 14 times. This variable has $26,083(46.73 \%)$ missing values (see Table 5.4 ). This is a very large number of missing values(near 50\%. Most of these values are likely to be zero. As this is a key explantory variable imputation will be carried out for parity in Chapter 7. For further analysis work parity has been grouped into two categories, one for mothers who have never given birth before and the second for mothers who have given birth at least once before.

Table 5.4: Frequency of Parity

\begin{tabular}{|l|r|}
\hline Parity & Number of Mothers \\
\hline 0 & $19,014(34.07 \%)$ \\
1 & $5,774(10.35 \%)$ \\
2 & $2,950(5.29 \%)$ \\
3 & $1,115(2.00 \%)$ \\
4 & $484(0.87 \%)$ \\
5 & $212(0.38 \%)$ \\
6 & $104(0.19 \%)$ \\
7 & $39(0.07 \%)$ \\
8 & $21(0.04 \%)$ \\
9 & $8(0.01 \%)$ \\
$10+$ & $7(0.01 \%)$ \\
Missing & $26,083(46.73 \%)$ \\
\hline Total & $55,811(100 \%)$ \\
\hline
\end{tabular}

\section{Parity by Age Group}

For mothers with known parity, age by parity in figure 5.9 shows $98 \%$ of mothers who have only given birth once for age group 19 and under. $93 \%$ of mothers aged 20-24 have only given birth once. There are more mothers in the older age groups, 35-39 and 40+ that have given birth more than once. A test of association shows that there is a relationship between parity and mothers age $\left(\chi^{2}=4002, \mathrm{df}=5, \mathrm{p}\right.$-value $\left.<0.0001\right)$. 


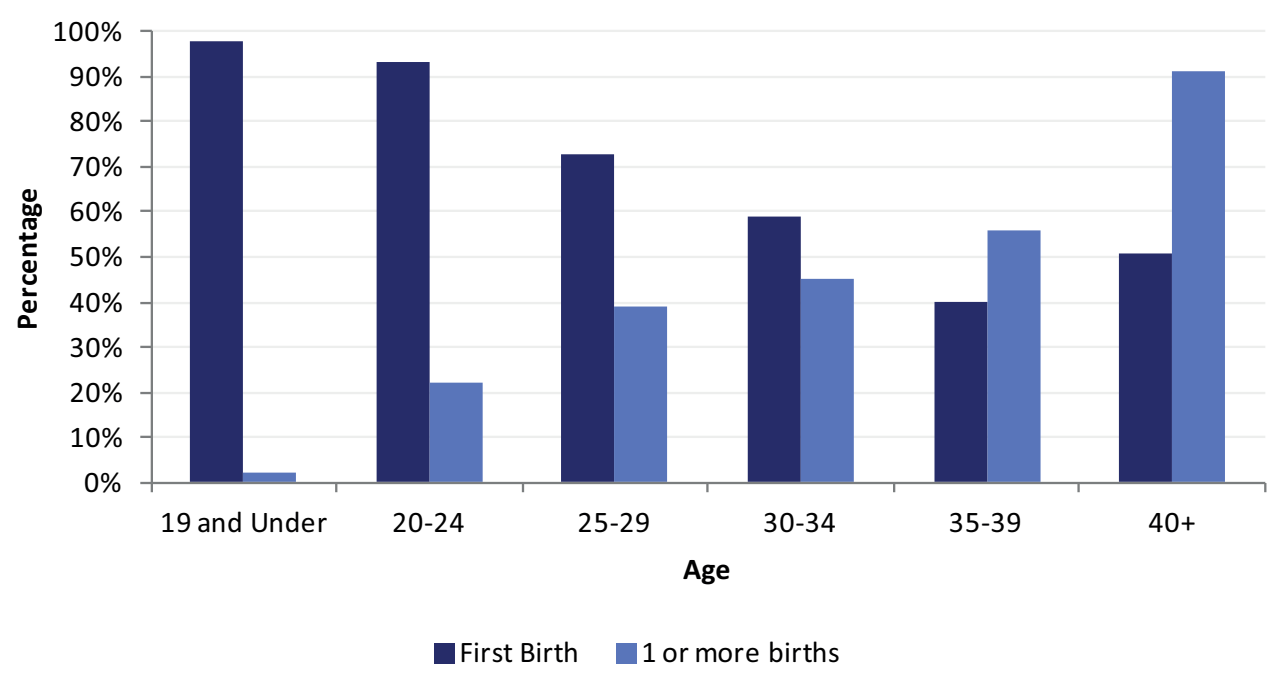

Figure 5.9: Age by Parity for all Mothers

\section{Parity by Deprivation Index}

Parity by Deprivation Index shows for all deprivation indices excluding index 10 , over $60 \%$ of mothers with known parity are first time mothers. Deprivation Index 2 only reports $57 \%$ of mothers giving birth for their first time and correspondingly a higher proportion of mothers who have previously given birth at $43 \%$, see figure 5.10 . The findings of higher rates of one or more prior births at higher deprivation indices is consistent with the ealrier finding that birth rates are higher at higher deprativation indices. A test of association shows that there is a relationship between parity and deprivation index $\left(\chi^{2}=114, \mathrm{df}=9\right.$, $\mathrm{p}$-value $\left.<0.0001\right)$.

\subsubsection{Birth Interventions and mode of delivery}

\section{Birth Interventions}

There are some common birth inventions that are used during child birth. These can include induction, epidural, episiotomy and manual removal of 


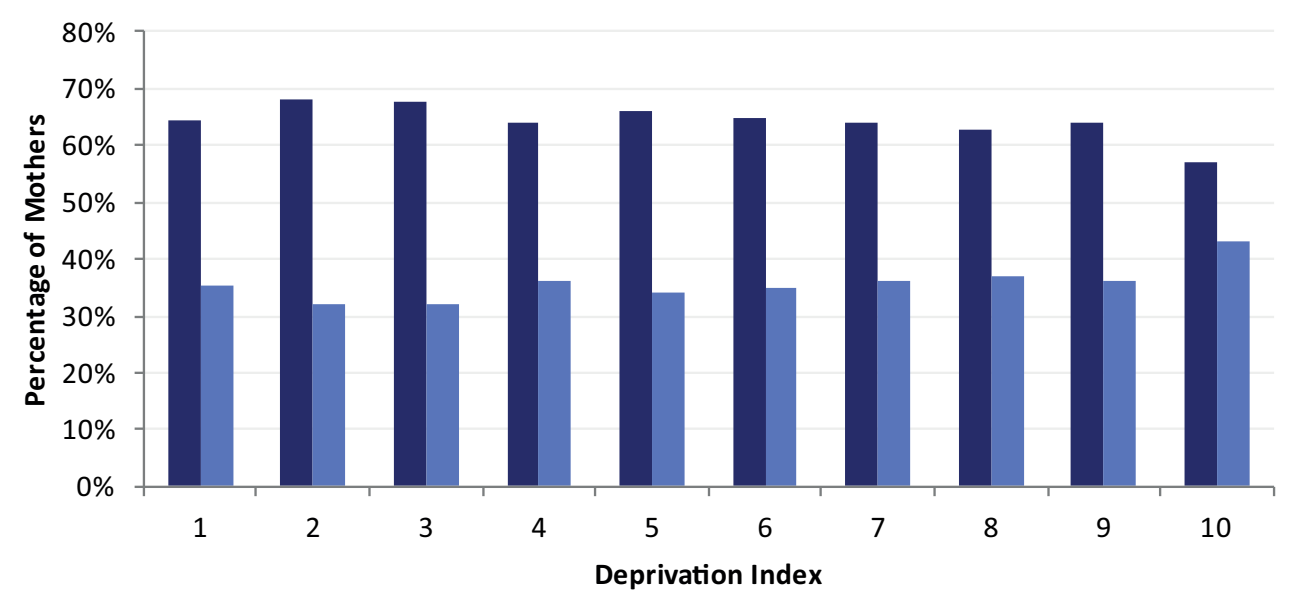

Girst Birth 1 or more births

Figure 5.10: Deprivation Index by Parity

the placenta. Episiotomy and manual removal of the placenta are two important factors for PPH (University of Virginia Health System, 2010; Harrison, 1998). In the NMDS 5,553 (9.95\%) of mothers had episiotomy and $810(1.45 \%)$ had a manual removal of the placenta.

\section{Mode of Delivery}

There are many medical tools that can assist the mother with delivery. Not all mothers require these procedures and are therefore classified as a 'normal birth'. There are large numbers of emergency Caesarean sections $(7,732)$ and Elective Caesarean Sections $(5,732$, see Table 5.5). There are small proportions of other assisted births such as breech, the use of forceps or vacuum extraction. $66.46 \%(37,093)$ of mothers had a normal birth. Caesarean sections accounted for $24.12 \%$, the remaining are breech births $(0.25 \%)$, forceps $(3.17 \%)$ and vacuum extraction $(6.00 \%)$, (see Table 5.5). There are 5 different categories of breech births these are: an assisted breech delivery, assisted breech delivery with forceps to aftercoming head, a breech extraction, a breech extraction with forceps to after- 
coming head and a spontaneous breech delivery. As birth numbers are very low, these have been combined to one category called breech.

Table 5.5: Type of Births Procedures

\begin{tabular}{|l|r|}
\hline Type of Birth & Number of Births \\
\hline Emergency Caesarean Section & $7,732(13.85 \%)$ \\
Elective Caesarean Section & $5,732(10.27 \%)$ \\
Breech & $137(0.25 \%)$ \\
Forceps & $1,768(3.17 \%)$ \\
Vacuum Extraction & $3,349(6.00 \%)$ \\
Normal Birth & $37,093(66.46 \%)$ \\
\hline Total & $55,811(100 \%)$ \\
\hline
\end{tabular}

\subsection{Potential Predictors for PPH}

This section looks at potential factors that can be related to PPH. As this dataset does not contain all factors that are associated a few variables have been selected to be investigated. These variables are most likely to have potential impacts on PPH. First a summary of the number of PPH for each DHB will be presented, then demographic, maternal, birth characteristics and birth interventions are explored.

\subsubsection{District Health Boards}

Table 5.6 shows in order of highest proportion of PPH for each DHB. This shows Auckland DHB has the highest proportion of PPH at $14.02 \%$, followed by Otago with the next highest proportion at $11.19 \%$. Tairawhiti at $11.09 \%$, Northland at $11.32 \%$, Mid Central, Counties Manukau and Waikato at $10 \%$. Nelson Marlborough and Southland DHBs have very small proportions $4.25 \%$ and $3.90 \%$ respectively. The national rate of $\mathrm{PPH}$ is $9.40 \%$. 
This table 5.6 shows where DHBs sit amongst the national rate of PPH. It shows that there are some much higher rates $(14.02 \%)$ than the national rate and some very low rates (3.90\%). Even though Tairawhiti has a very low number of births, it has a high rate of $\mathrm{PPH}$ at $11.09 \%$, compared to the national rate. Where as Waitemata has a high number of births $(6,797)$ but a lower rate of $\mathrm{PPH}$ at $7.55 \%$. A test of association shows that there is a relationship between PPH and DHBs $\left(\chi^{2}=369, \mathrm{df}=20, \mathrm{p}\right.$-value $\left.<0.0001\right)$.

Table 5.6: The number of Mothers who have had a PPH by DHB

\begin{tabular}{|l|r|r|r|r|}
\hline \multirow{2}{*}{ DHB } & \multicolumn{4}{|c|}{ Postpartum Haemorrhage } \\
\cline { 2 - 5 } & Yes & No & Total & $(\%)$ \\
\hline Auckland & 854 & 5,237 & 6,091 & 14.02 \\
Otago & 210 & 1,666 & 1,876 & 11.19 \\
Tairawhiti & 72 & 577 & 649 & 11.09 \\
Northland & 204 & 1,646 & 1,850 & 11.03 \\
Counties Manukau & 835 & 7,051 & 7,886 & 10.59 \\
MidCentral & 211 & 1,793 & 2,004 & 10.53 \\
Waikato & 488 & 4,269 & 4,757 & 10.26 \\
Canterbury & 593 & 5,446 & 6,039 & 9.82 \\
West Coast & 30 & 310 & 340 & 8.82 \\
Taranaki & 118 & 1,312 & 1,430 & 8.25 \\
Capital and Coast & 284 & 3,227 & 3,511 & 8.09 \\
Hutt & 155 & 1,763 & 1,918 & 8.08 \\
Lakes & 110 & 1,270 & 1,380 & 7.97 \\
Waitemata & 513 & 6,284 & 6,797 & 7.55 \\
Hawkes Bay & 149 & 1,837 & 1,986 & 7.50 \\
Bay of Plenty & 186 & 2,430 & 2,616 & 7.11 \\
Wairarapa & 31 & 424 & 455 & 6.81 \\
South Canterbury & 41 & 561 & 602 & 6.81 \\
Whanganui & 49 & 734 & 783 & 6.26 \\
Nelson Marlborough & 63 & 1,419 & 1,482 & 4.25 \\
Southland & 53 & 1,306 & 1,359 & 3.90 \\
\hline Total & 5,249 & 50,562 & 55,811 & 9.40 \\
\hline
\end{tabular}




\subsubsection{Demographics}

This sections investigates mothers' demographic characteristics such as age and ethnicity against PPH.

\section{Mother's Age}

Table 5.7 shows the percentage of PPH by each age group. This shows the age group $40+$ years has the highest percentage of $\mathrm{PPH}$ at $10.87 \%$. This is approximately $2 \%$ higher than the 19 and under age group which has the lowest percentage of $\mathrm{PPH}$ at $8.67 \%$. All other age groups have a rate which is close to the $\mathrm{PPH}$ national rate of $9.40 \%$. A chi-square test shows there is no relationship between age and $\mathrm{PPH}\left(\chi^{2}=8.46, \mathrm{df}=5, \mathrm{p}\right.$-value $\left.=0.1324\right)$.

Table 5.7: Mother's Age by PPH

\begin{tabular}{|l|r|r|r|r|}
\hline \multirow{2}{*}{ Age } & \multicolumn{4}{|c|}{ Postpartum Haemorrhage } \\
\cline { 2 - 5 } & Yes & No & Total & $(\%)$ \\
\hline 19 and Under & 387 & 4,075 & 4,462 & $8.67 \%$ \\
$20-24$ & 933 & 8,793 & 9,726 & $9.59 \%$ \\
$25-29$ & 1,263 & 12,274 & 13,537 & $9.33 \%$ \\
$30-34$ & 1,505 & 14,527 & 16,032 & $9.39 \%$ \\
$35-39$ & 939 & 9,073 & 10,012 & $9.38 \%$ \\
$40+$ & 222 & 1,820 & 2,042 & $10.87 \%$ \\
\hline Total & 5,249 & 50,562 & 55,811 & $9.40 \%$ \\
\hline
\end{tabular}

\section{Mother's Ethnicity}

Figure 5.11 shows the percentage of mothers ethnic groups for mothers who have had a PPH. The European group shows that $8.52 \%$ of mothers had a PPH. The Pacific ethnic group has the highest percentage of mothers who have had a PPH at $13.62 \%$. Even though Pacific ethnicity makes up a total of $10.23 \%$ of the population of mothers who gave birth in 2007 , a high proportion of these mothers had a PPH.

Even though European is the biggest ethnic group (58\%, see table, 5.3) the 
number of mothers have a lower rate of $\mathrm{PPH}$ at $8.52 \%$ compare this to $13.62 \%$ for the Pacific group. The Asian ethnicity have the second highest rate of $\mathrm{PPH}$ at $10.84 \%$, and Māori at $9.14 \%$. For a test of association there is a relationship between PPH and ethnicity $\left(\chi^{2}=162, \mathrm{df}=4, \mathrm{p}\right.$-value $<$ 0.0001).

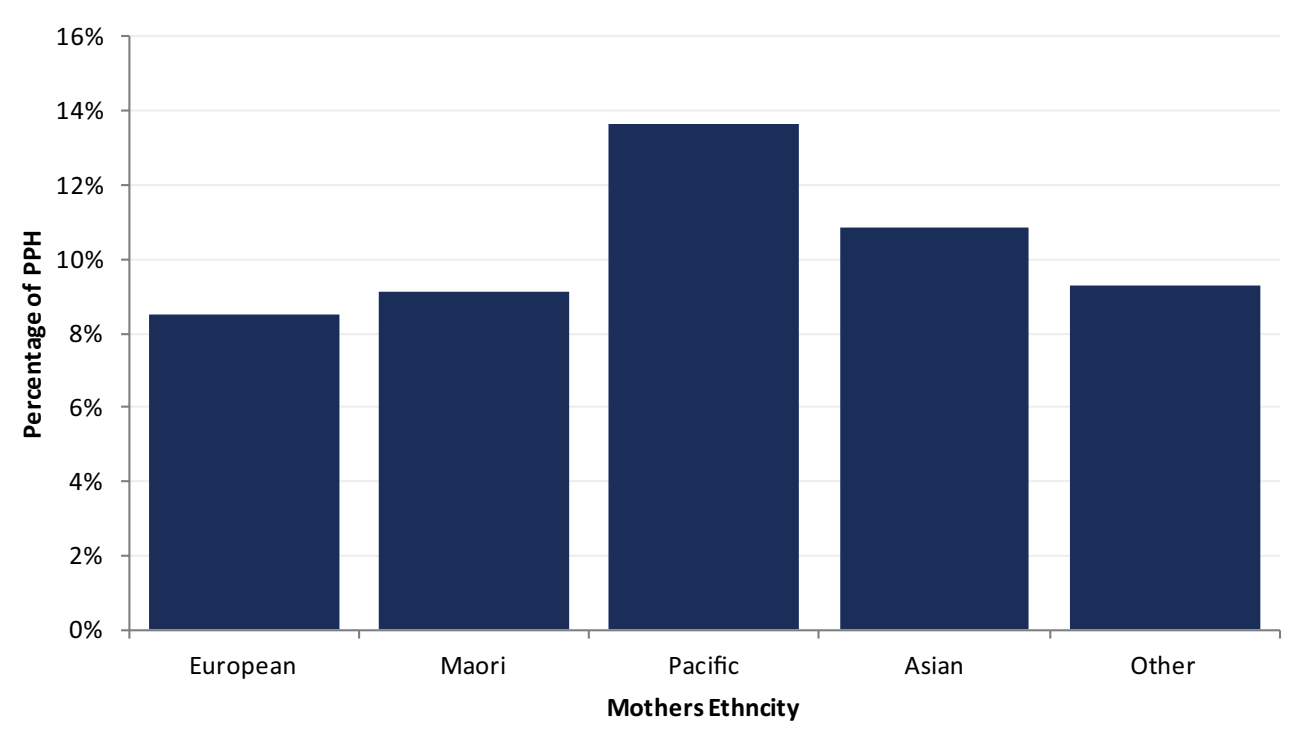

Figure 5.11: Mother's Ethnicity for mothers who have had a PPH

\subsubsection{Maternal Birth Characteristics}

This section investigates maternal birth characteristics such as parity, birth weight, gestation, episiotomy and removal of placenta against $\mathrm{PPH}$.

\section{Parity}

Looking at parity against PPH there seem to be more first time mothers that have had a PPH, (11.07\%) compared to mothers who have given birth at least once before $(8.81 \%$, see table 5.8$)$. Note that there are 26,083 missing values for this variable. If all observations were complete, this may change 
the percentage of mothers who have a PPH. A test of association shows a relationship between parity and $\mathrm{PPH}\left(\chi^{2}=38.02, \mathrm{df}=1, \mathrm{p}\right.$-value $\left.<0.0001\right)$.

Table 5.8: Parity by PPH

\begin{tabular}{|l|l|l|l|l|}
\hline \multirow{2}{*}{ Parity } & \multicolumn{3}{|c|}{ Postpartum Haemorrhage } \\
\cline { 2 - 5 } & Yes & No & Total & $(\%)$ \\
\hline 0 & 2,105 & 16,909 & 19,014 & 11.07 \\
$1+$ & 944 & 9,770 & 10,714 & 8.81 \\
Missing & 2,200 & 23,883 & 26,083 & 8.43 \\
\hline Total & 5,249 & 50,562 & 55,811 & 9.40 \\
\hline
\end{tabular}

\section{Birth Weight}

The average birth weight for the NMDS for 2007 is 3466g. Any weight under $2500 \mathrm{~g}$ is considered a low birth weight. For mothers who have had a $\mathrm{PPH}, 88 \%$ of birth weights range between $2500 \mathrm{~g}$ to $4499 \mathrm{~g}$. There are smaller percentage of babies weighing $2499 \mathrm{~g}$ or less (5\%) and $7 \%$ weight over $4500 \mathrm{~g}$. see table 5.9 A Chi-Square test of association shows that there is a difference between PPH and babie's birth weights $\left(\chi^{2}=144.12, \mathrm{df}=2\right.$, $\mathrm{p}$-value $<0.0001)$.

Table 5.9: New Born's birth weight for mothers who had a PPH

\begin{tabular}{|l|r|}
\hline Birth weight & Frequency \\
\hline$<2499$ & $256(5 \%)$ \\
$2500-4499$ & $4,660(88 \%)$ \\
$4500+$ & $292(7 \%)$ \\
\hline Total & $5,208(100 \%)$ \\
\hline
\end{tabular}

\section{Gestation}

The proportion of pre-term mothers who have a $\mathrm{PPH}$ is $9.38 \%$ and the proportion of post-term mothers who have a $\mathrm{PPH}$ is higher at $15.71 \%$. See table 5.10.A test of association shows that there is no difference between 
gestation and $\mathrm{PPH}\left(\chi^{2}=3.25, \mathrm{df}=2, \mathrm{p}\right.$-value $\left.=0.1960\right)$.

Table 5.10: PPH by Gestation

\begin{tabular}{|l|r|r|r|r|}
\hline Gestation & Yes & No & Total & $(\%)$ \\
\hline Pre term (<37 weeks) & 556 & 5,369 & 5,925 & 9.38 \\
Normal (37-42) & 4,596 & 44,211 & 48,807 & 9.42 \\
Post-term (42+ weeks) & 11 & 59 & 70 & 15.71 \\
Missing & 86 & 923 & 1,009 & 8.52 \\
\hline Total & 5,249 & 50,562 & 55,811 & 9.4 \\
\hline
\end{tabular}

\section{Episiotomy and Removal of Placenta}

A test of association shows us that there is an association between episiotomy and PPH $\left(\chi^{2}=152.31, \mathrm{df}=1, \mathrm{p}\right.$-value $\left.<0.0001\right)$. Of the 5,553 mothers who had episiotomy 777 of these had a PPH (14.8\%). For mothers who had the procedure to remove the placenta (810) 574 of these mothers had a PPH (70.8\%). A chi-square test shows that there is an association between removal of the placenta and PPH $\left(\chi^{2}=3643.74, \mathrm{df}=1\right.$, p-value $<0.0001)$.

\subsubsection{Mode of Delivery and birth interventions}

This section explores mode of delivery and birth interventions against PPH.

Table 5.11 shows the percentage of mothers who have had a PPH for the different types of birth. The use of forceps has a high percentage of PPH at $16.91 \%$ and for Emergency Caesarean Sections the rate is $15.11 \% .14 .27 \%$ of mothers who have a Vacuum Extraction have a PPH. 7.43\% of mothers who had a normal birth have a PPH. This is a lower rate than the national average $(9.40 \%)$. A chi-square test for a test of association shows that there is an relationship between the type of birth and $\mathrm{PPH}\left(\chi^{2}=676.45, \mathrm{df}=9\right.$, 
p-value $<0.0001)$

Table 5.11: Type of Birth by Postpartum Haemorrhage

\begin{tabular}{|l|r|r|r|r|}
\hline \multirow{2}{*}{ Type of Birth } & \multicolumn{4}{|c|}{ Postpartum Haemorrhage } \\
\cline { 2 - 5 } & Yes & No & Total & $(\%)$ \\
\hline Emergency Caesarean Section & 1,168 & 6,564 & 7,732 & 15.11 \\
Elective Caesarean Section & 533 & 5,199 & 5,732 & 9.30 \\
Breech & 14 & 123 & 137 & 10.22 \\
Forceps & 299 & 1,469 & 1,768 & 16.91 \\
Vacuum Extraction & 478 & 2,871 & 3,349 & 14.27 \\
Normal Birth & 2,757 & 34,336 & 37,093 & 7.43 \\
\hline Total & 5,249 & 50,562 & 55,811 & 9.40 \\
\hline
\end{tabular}




\section{Chapter 6}

\section{Unadjusted Comparison}

In this chapter we explore the DHB characteristics, PPH rates, ethnicity, age, gender of the baby, parity and deprivation index. The second section of this chapter presents the method and results for the unadjusted funnel plot.

\subsection{DHB Comparisons}

From the EDA we already know that DHBs have different population sizes, birth rates and PPH rates (see figure 6.1). As DHBs are an important part of this analysis the following section explores demographic and maternal characteristics of DHBs.

These figures are presented in geographical order from the top of the North Island to the bottom of the South Island. If the figures were to be presented in alphabetical order, the pattern of similarities and differences between adjacent DHBs would be lost. Therefore representing these figures in a geographical order helps gain a clearer understanding of the relationships between DHBs (see figure 5.1). 


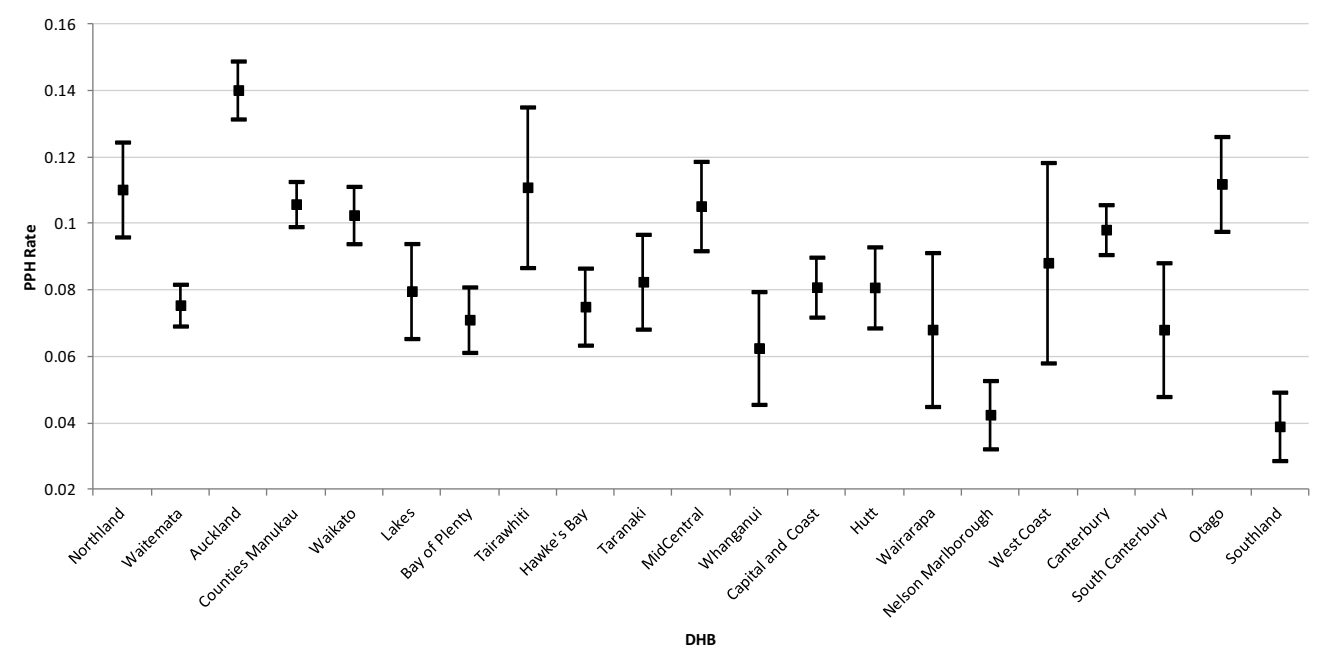

Figure 6.1: PPH comparison across DHBs

First we look at the PPH rate for each DHB and their 95\% confidence intervals (see figure 6.1). The confidence intervals are calculated as: $\hat{\theta}_{i} \pm$ $Z \sqrt{\frac{\hat{\theta}_{i}\left(1-\hat{\theta}_{i}\right)}{n_{i}}}$ where $\hat{\theta}_{i}=X_{i} / n_{i}$, and $X_{i}=$ the number of PPH for DHB $i$ and $n_{i}=$ the total number of births for DHB $i$ and $Z=1.96$.

The PPH rate differs amongst DHBs. Auckland DHB has the highest rate at $14 \%$, with very narrow confidence limits. Where as Tairawhiti and the West Coast have very wide confidence limits due to their smaller populations. Nelson Marlborough and Southland DHBs have the lowest PPH rate at 0.038 and 0.042 respectively .

\subsubsection{Ethnicity}

From figure 6.2 shows that Northland, Lakes and Tairawhiti DHBs have the largest proportions of Māori ethnicity at $46 \%$, Lakes $45 \%$ and Tairawhiti $59 \%$. Whanganui (35\%), Hawke's Bay (34\%), Bay of Plenty (32\%) and Waikato (28\%) have the next largest proportions of Māori. Auckland at 


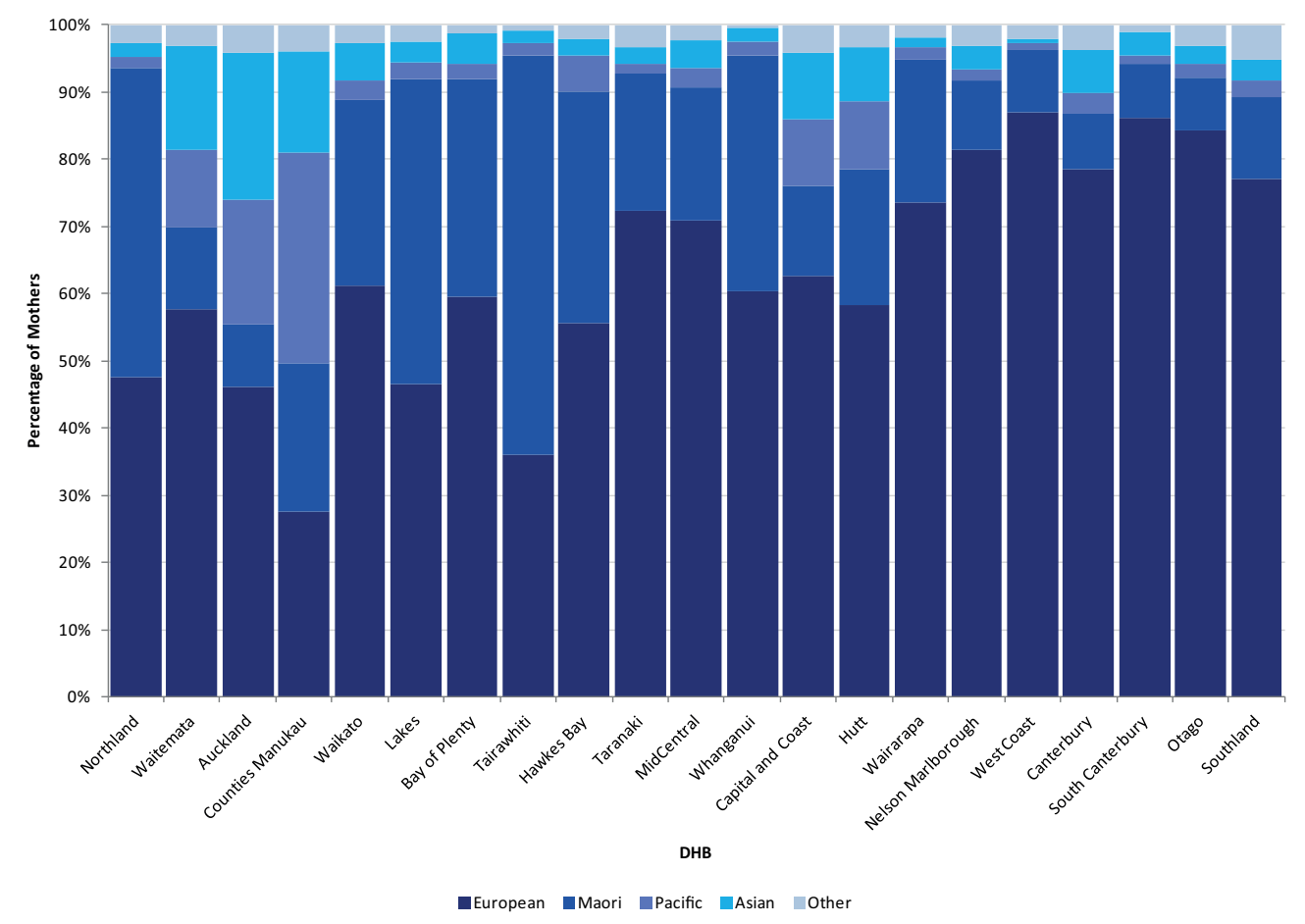

Figure 6.2: DHB by Ethnicity

$9 \%$, Canterbury, South Canterbury and Otago at $8 \%$ have the smallest proportions of Māori.

West Coast (87\%), South Canterbury (86\%), Otago (84\%) and Nelson Marlborough $(81 \%)$ have the highest proportion of European ethnicity. Counties Manukau (27\%) and Tairawhiti (36\%) have the smallest proportion of European ethnicity.

Counties Manukau has the largest proportion of Pacific ethnicity at 31\% followed by Pacific ethnicity are Auckland (19\%), Waitemata (12\%), Capital \& Coast and Hutt (10\%). Taranaki, West Coast and South Canterbury have the lowest proportions of Pacific ethnicity at $1 \%$. 
Auckland has the highest proportion of Asian ethnicity at 22\%, followed by Waitemata and Counties Manukau at $15 \%$ and Capital \& Coast at $10 \%$. Wairarapa and West Coast have the lowest proportions of Asian ethnicity at $1 \%$.

Overall we can see that the top of the North Island is largely made up of European, Māori, Pacific and Asian ethnicities. The middle of the North Island has the highest proportion of Māori (this area includes Lakes, Bay of Plenty, Tairawhiti, Hawke's Bay, Taranaki and MidCentral). The lower part of the North Island is predominately made up of European ethnicity and approximately 18\% Māori. This area consists of Whanganui, Wairarapa, Hutt and Capital \& Coast DHBs. The South Island DHBs are largely made up of European ethnicity (80\%). Visually we can see that the DHBs are made up of different proportions of ethnic groups. A test of association shows that there is a relationship between DHBs and ethnicity, $\left(\chi^{2}=\right.$ 14617, $\mathrm{df}=80, \mathrm{p}$-value $<0.0001)$.

\subsubsection{Mother's Age}

Tairawhiti, Lakes and Whanganui DHBs have the youngest median age of mothers giving birth at 27 years. Capital \& Coast have the highest median age at 32 years. Waitemata, Auckland and Canterbury have the next highest median age of 31 years,(see figure 6.3). The rest of the DHBs have a median of 28,29 or 30 years.

The median has been used here to identify the 'middle' age, rather than the average. The median is used as a measure of location in the skewed distributions. For example if one DHB had extremely low values then the mean would move towards these values. This would skew the mean and would produce biased results. 


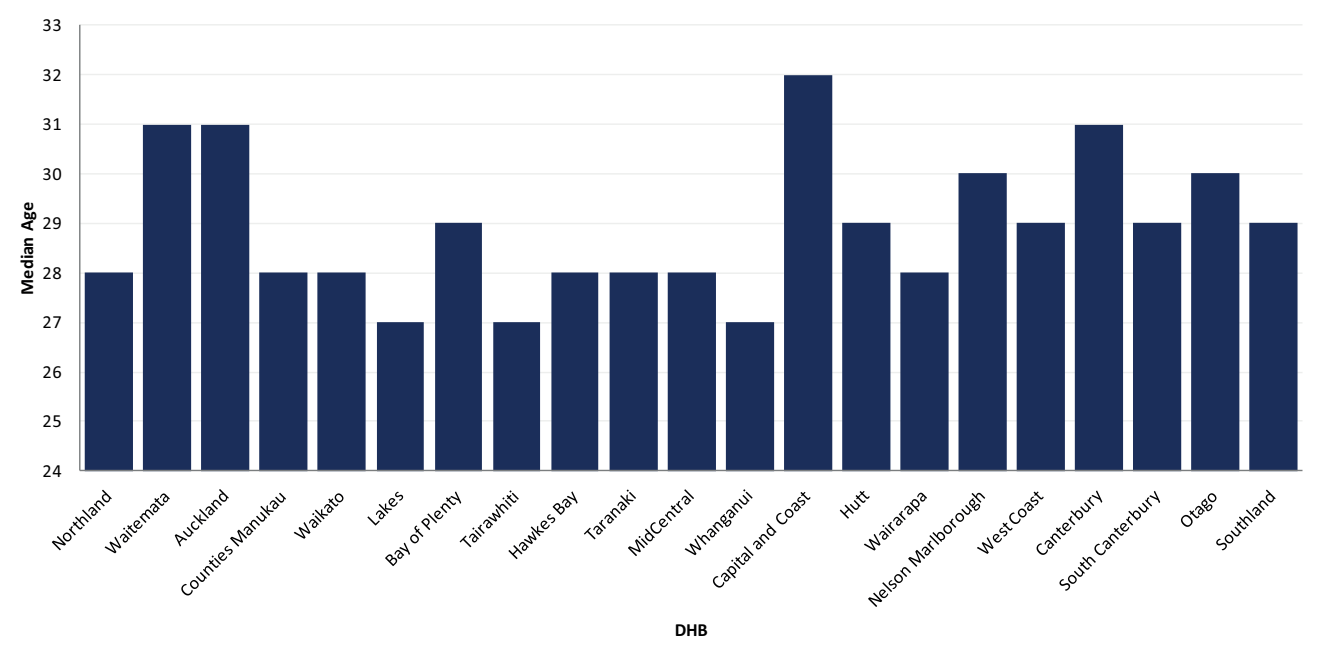

Figure 6.3: DHB by Median Age

For a test of association age has been categorised into age groups. The age groups used here are $<19,20-24,25-29,30-34,35-39$ and 40+. A test of association shows that there is a relationship between DHB and age $\left(\chi^{2}=1979, \mathrm{df}=100, \mathrm{p}\right.$-value $\left.<0.0001\right)$.

\subsubsection{Gender}

There are $51.5 \%$ male babies and $48.5 \%$ female babies. Auckland (52.52\%), Bay of Plenty (52.14\%), Capital \& Coast (52.49\%) and South Canterbury $(53.32 \%)$ have the highest proportion of male births. A test of association shows no relationship between gender and DHB $\left(\chi^{2}=15, \mathrm{df}=20\right.$, $\mathrm{p}$-value $=0.7498)$. 


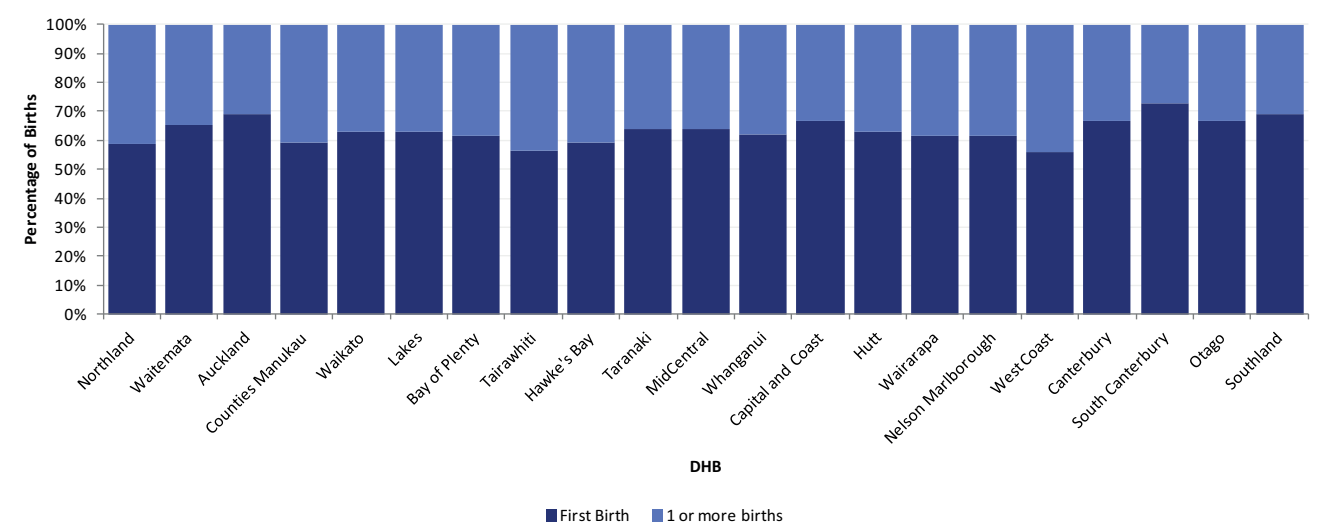

Figure 6.4: DHB by Parity

\subsubsection{Parity}

Figure 6.4 shows there are more first time mothers for all DHBs. South Canterbury (72.70\%), Southland (68.92\%), Auckland (68.88\%), Canterbury $(66.77 \%)$ and Otago $(66.70 \%)$ have the highest percentage of first time mothers. There are high proportions of mothers who have had previous births in West Coast (43.89\%), Tairawhiti (43.70\%) and Northland (41.35\%). This is using the raw data and not the imputed values. There are approximately 26,083 missing values. A test of association shows that there is a relationship between parity and $\operatorname{DHB}\left(\chi^{2}=158, \mathrm{df}=20\right.$, $\mathrm{p}$-value $\left.<0.0001\right)$.

\subsubsection{Deprivation Index}

Figure 6.5 shows the median deprivation index for Northland, Counties Manukau, Lakes, Tairawhiti and Hawke's Bay DHB is 9. The least deprived areas with an index of 4 is Capital \& Coast. Waitemata, Canterbury, Otago and Southland have a median deprivation index of 5. A chi-square test shows an association between deprivation index and DHB $\left(\chi^{2}=19484, \mathrm{df}=180, \mathrm{p}\right.$-value $\left.<0.0001\right)$. 


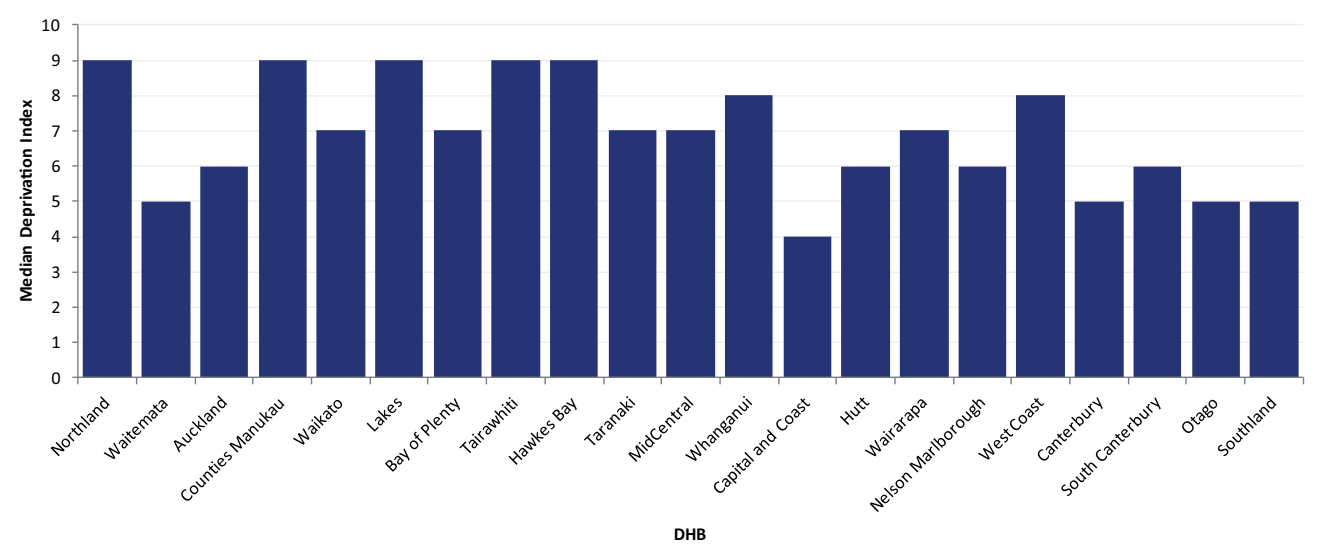

Figure 6.5: DHB by NZ Deprivation Index

\subsubsection{Summary}

Results from the EDA show a variety of characteristics of key explanatory factors between DHBs. DHBs have different population mixes, ethnic groups, deprivation indexes and birth characteristics. Northland DHB has the largest Māori population ( $46 \%$ ) with $41.35 \%$ of mothers having previously given birth. Northland DHB is also one of the most deprived areas with a median index of 9. Tairawhiti DHB also has one of the largest Māori population (46\%), with median age of mothers giving birth of 27 years and $43.7 \%$ of mothers who have previously given birth. Tairawhiti DHB also has a deprivation index of 9. Capital \& Coast has the highest median age of mothers giving birth at 32 years and is the lowest deprived area at index 4 . South Canterbury has the largest European ethnic group at $86 \%$ and $73 \%$ are first time mothers. This shows us that DHBs differ in populations mix across NZ. These differences must be adjusted for when comparing PPH rates across DHBs since some of these demographic and birth characteristics are related to PPH. We will start considering a comparison with no adjustment. 


\subsection{Unadjusted Funnel Plot}

For the unadjusted funnel plot the rate of PPH for each DHB is calculated as the total number of women who have had a PPH divided by the total population of mothers for that DHB. This is the PPH rate. Each DHB has their own specific rate of $\mathrm{PPH}$. This $\mathrm{PPH}$ rate is then plotted against the number of women who gave birth in 2007 for the DHB. The rate $\theta$ is the NZ national rate of $\mathrm{PPH}$ and $\theta_{0}$ is the target rate. The control limits are calculated for each DHB and superimposed onto the graph. The $95 \%$ and $99.8 \%$ control limits are calculated for each DHB and these are then plotted as the control limits.

\subsubsection{Unadjusted Funnel Plots Method}

The unadjusted funnel plot is constructed using the follow method described below.

1. Calculate the unadjusted rate $\hat{\theta}_{i}=X_{i} / n_{i}$ where $X_{i}$ is the total number of women who have had a PPH in DHB $i$ and $n_{i}$ is the total number of women who gave birth in DHB $n_{i}$. This is the proportion of women that have had a PPH in each DHB.

2. Calculate the variance using the equation $\operatorname{var}_{i}=\hat{\theta_{0}}\left(1-\hat{\theta_{0}}\right) / n_{i}$ where $\hat{\theta_{0}}=y_{i} / n_{i}$ where $\hat{\theta_{0}}=\sum_{i} X_{i} / \sum_{i} n_{i}$ is the national rate and $n_{i}$ is the total number of women who give birth in DHB $i$.

3. The confidence interval can be calculated as $\hat{\theta}_{0} \pm Z_{p} \sqrt{v a r_{i}}$

4. To plot the unadjusted funnel plot. Plot $\hat{\theta_{0}}=\frac{Y_{i}}{n_{i}}$ against the precision parameter $n_{i}$, with the target $\hat{\theta}_{0}=p$ shown as a horizontal line and superimpose the control limits on the plot as function of the parameter $n_{i}$. The national rate is $\theta_{0}=5249 / 55811=0.09405$. The horizontal 
axis is the DHB population and the vertical axis the PPH rate. Each DHB's unadjusted PPH rate is then plotted and the control limits superimposed on top of this.

\subsubsection{Unadjusted Funnel Plot Results}

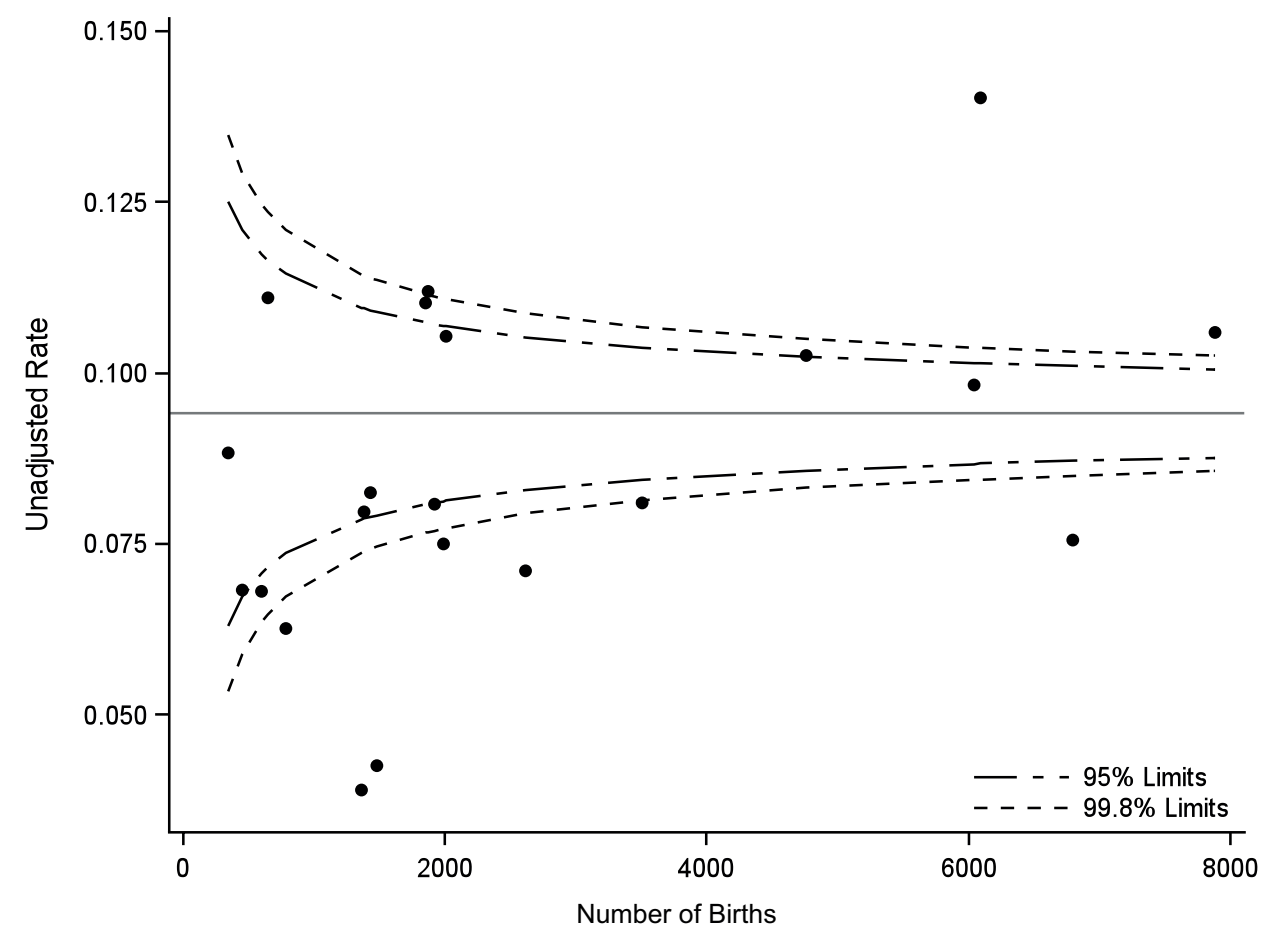

Figure 6.6: Unadjusted Funnel Plot

Figure 6.6 shows the unadjusted funnel plot for the rate of $\mathrm{PPH}$ for each DHB and the control limits. Of the 21 DHBs, 7 are within the $95 \%$ control limits and 14 are outside these control limits. There are 11 DHBs that are within the $99.8 \%$ confidence limits, with two of these DHBs on the boarder of the $95 \%$ confidence limits. Those DHBs that sit above the upper control limits are underperfomring, with a higher than expected proportion of PPH. Those that sit below the lower control limits have significantly lower 
PPH rates than that seen at the national level. The funnel plot show there are some extreme outliers at both ends of the spectrum. In the upper right of the funnel plot one DHB that has an unadjusted rate over 0.125 and is far beyond the control limits. The lower right hand of the figure shows two DHBs that have an unadjusted rate under 0.05 and are well outside the control limits, even when the control limits here are wider at this point.

The funnel plot shows large variation between the PPH rates across DHBs. This variability shown in figure 6.6 could be because of unmeasured covariates. As the funnel plot shows extensive overdispersion, the next step would be to adjust for covariates. This would include demographics, maternal and birth characteristics. 


\section{Chapter 7}

\section{Risk Adjusted Comparisons}

In this chapter the imputation method and results are presented. The choice of model for the logistic regression is shown. The risk adjusted and winsorised funnel plots methods and results are presented.

\subsection{Imputation}

This section explores the variables to impute, describes the method used and the results of the imputation are presented.

\subsubsection{Investigation of Variables to be Imputed}

To investigate if imputation is a valid procedure we look at the distributions of the variables to be imputed. This is to identify how they differ from the rest of the sample. The three variables to be investigated are gestation, parity and birth weight. These have been identified as important predictors of PPH (University of Virginia Health System (2010); Taylor et al. (2005); Waitemata District Health Board (2006); Jacobs (2012); Combs et al. (1991)) and also have a large number of missing values as seen in the EDA. Gestation has 1.84\% (1,009) missing values, Birth weight has $0.8 \%$ 
(448) missing values and parity has $46.74 \%(26,083)$ missing values. Ideally we should not see any difference in distributions for the predicators against the outcome (except for the data size generated difference). If the data is MAR then these distributions should look relatively similar.

First we look at the variables and their distributions against age, ethnic group and deprivation index. Then the missing values are investigated against PPH and DHBs to see how they differ. For example, a distribution of mothers age for all mothers who gave birth in 2007 will be shown and this will be compared against mothers who have a missing recorded value for gestation.
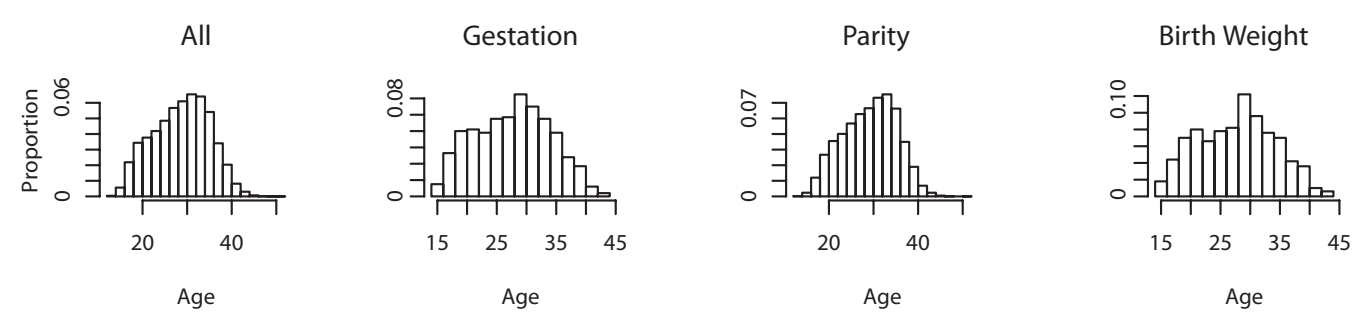

Figure 7.1: Age Distribution, for the whole dataset, data where gestation is missing, data where parity is missing and data where birth weight is missing (as defined below)

The first investigation shows the distributions of age, ethnic group and deprivation index against gestation, parity and birth weight. There are four data sets used for the first comparison against age. In figure 7.1 the 'all' distribution shows mothers age for all mothers who gave birth in 2007. The 'gestation' distribution shows gestation by mothers age for mothers that have a missing value for gestation. The 'Parity' histogram shows the distribution of mothers age for mothers who have a missing value for parity. The 'birth weight' histogram shows mothers age by birth weight for mothers who have a missing value for birth weight. 
For the age distribution against the missing variables (see figure 7.1) the distributions of parity displays a similar pattern to the whole dataset. Gestation is more 'flat' between the ages of 18-26 years rather than a gradual increased step by step to the peak age of 30 years. Birth weight also has a more 'flat' distribution of ages between 15-25 years. The peak here is 30 years which is the same for all mothers age. Even though gestation and birth weight show a slightly different pattern of ages in the younger years from 15-25, the overall pattern is still similar to the entire dataset.

Figure 7.2 shows the distribution of ethnic group and deprivation index for the entire dataset and where gestation, parity and birth weight values are missing. Ethnic group is coded as 1 for European, 2 for Māori, 3 for Pacific, 4 for Asian and 5 for Other ethnicity. The trend for the ethnicity is similar for all four histograms. It shows the largest group is European, followed by Māori. Where gestation has a missing value the Māori ethnic group have a higher number missing values proportionally to the other ethnic groups. The birth weight figure also shows a similar pattern. However the pattern for ethnicity is relatively similar for gestation, parity and birth weight against the entire dataset. This shows that the missingness is not related to these characteristics.

In the deprivation index comparison (see figure 7.2), all three distributions of gestation, parity and birth weight have a similar pattern to the entire dataset. There are fewer mothers in the least deprived areas and more mothers in the most deprived areas. The gestation and birth weight histograms have a smaller amount of mothers in the least deprived areas compared to the entire dataset.

The second investigation looks at $\mathrm{PPH}$ and $\mathrm{DHB}$ and their distribution of missingness. PPH and DHBs are important measures in this study. It is therefore essential to explore the pattern of missingness for gestation, par- 


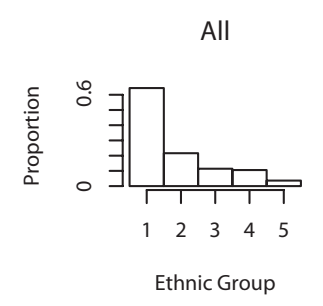

All

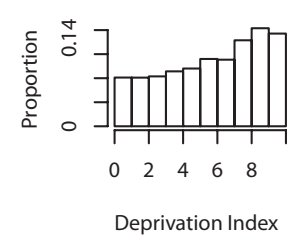

Gestation

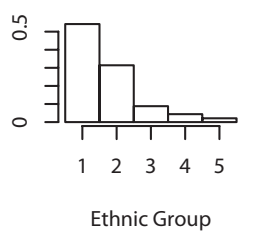

Gestation

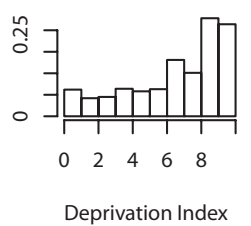

Parity

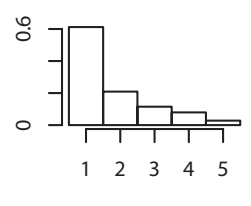

Ethnic Group

Parity

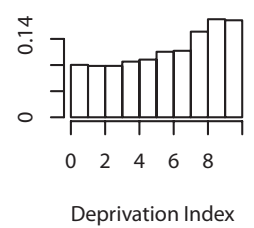

Birth Weight

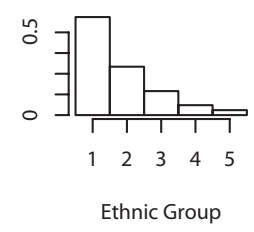

Birth Weight

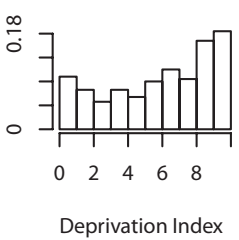

Figure 7.2: Distribution of Ethnic Group (top) and Deprivation Index distribution (bottom) for the whole dataset, where gestation is missing, where parity is missing and where birth weight is missing

ity and birth weight against PPH and DHBs.

Table 7.1: The number of percentage of missing parity by PPH.

$$
\text { Parity }
$$

\begin{tabular}{l|rrr|r} 
PPH & Observed & Missing & Total & Missing Rate \\
\hline Yes & 3,049 & 2,200 & 5,249 & $41.9 \%$ \\
No & 26,729 & 23,833 & 50,562 & $47.1 \%$ \\
\hline Total & 29,778 & 26,033 & 55,811 & $46.6 \%$
\end{tabular}

\begin{tabular}{l|rrr|r}
\multicolumn{5}{|c}{ Birth Weight } \\
PPH & Observed & Missing & Total & Missing Rate \\
\hline Yes & 5,206 & 43 & 5249 & $0.82 \%$ \\
No & 50,157 & 405 & 50,562 & $0.80 \%$ \\
\hline Total & 55,363 & 448 & 55,811 & $0.80 \%$
\end{tabular}

Table 7.1 shows the missingess rate for each variable by PPH. We would expect to see the same amount of missingness against each variable for $\mathrm{PPH}$ and no PPH. Table 7.1 shows this that there are some differences 


\begin{tabular}{l|rrr|r}
\multicolumn{5}{c}{ Gestation } \\
PPH & Observed & Missing & Total & Missing Rate \\
\hline Yes & 5,163 & 86 & 5249 & $1.64 \%$ \\
No & 46,639 & 923 & 50,562 & $1.83 \%$ \\
\hline Total & 54,082 & 1,009 & 55,811 & $1.84 \%$
\end{tabular}

with the missing rate for Parity. The missing rate for parity by no PPH is $47.1 \%$ and for a $\mathrm{PPH}$ is $41.9 \%$ these two rates are different. Birth wiehgts missingess rate for No $\mathrm{PPH}$ and for a PPH are similar at $0.80 \%$ and $0.82 \%$ respectively. Gestation missingess rate is differs by $0.2 \%$.

Table 7.2: Percentage of missing records for Gestation, Birth Weight and Parity for each DHB.

\begin{tabular}{|l|r|r|r|r|}
\hline DHB & Total Observed & Parity \% & Gestation \% & Birth Weight \% \\
\hline Northland & 1,850 & 45.89 & 0.22 & 0.22 \\
Waitemata & 6,797 & 47.15 & 0.03 & 0.13 \\
Auckland & 6,091 & 42.32 & 0.16 & 0.21 \\
Counties Manukau & 7,886 & 45.27 & 0.77 & 0.77 \\
Waikato & 4,757 & 43.73 & 1.64 & 1.43 \\
Lakes & 1,380 & 47.03 & 0.14 & 0.14 \\
Bay of Plenty & 2,616 & 43.08 & 3.52 & 0.46 \\
Tairawhiti & 649 & 58.40 & 0.31 & 0.15 \\
Hawke's Bay & 1,986 & 45.27 & 27.09 & 2.87 \\
Taranaki & 1,430 & 43.92 & 2.10 & 1.61 \\
MidCentral & 2,004 & 48.95 & 0.50 & 0.40 \\
Whanganui & 783 & 47.77 & 0.51 & 0.51 \\
Capital and Coast & 3,511 & 43.58 & 3.28 & 3.28 \\
Hutt & 1,918 & 45.78 & 1.25 & 1.15 \\
Wairarapa & 455 & 52.53 & 0.44 & 0.22 \\
Nelson Marlborough & 1,482 & 48.65 & 0.27 & 0.27 \\
West Coast & 340 & 47.06 & 0.59 & 0.59 \\
Canterbury & 6,039 & 54.69 & 0.10 & 0.23 \\
South Canterbury & 602 & 38.54 & 0.66 & 0.66 \\
Otago & 1,876 & 53.57 & 0.16 & 0.43 \\
Southland & 1,359 & 51.21 & 1.18 & 1.18 \\
\hline
\end{tabular}

Table 7.2 shows the percentage of missing values for parity, gestation and birth weight for each DHB. $47 \%$ of parity is missing, this has the largest number of missing values. Tairawhiti DHB has the largest number of miss- 
ing parity (58\%). Canterbury, Otago and Wairarapa have over half of the values of parity missing (54\%, 53\%, 52\% respectively). South Canterbury has the lowest number of missing values for parity $(38 \%)$. In general the DHBs have a similar proportion of missing values for parity and there are only a few DHBs that stand out as being different to the other DHBs. Almost half the data values are missing for this variable and therefore is not ignorable. Parity is the number of times a mother has given birth. The values can range from $0-14$ with 0 being the most frequent. The median is 0 and mean is 0.6468 . We will most likely want to impute this value in the range of $0-2$.

Gestation has $1.8 \%$ of missing values. In table 7.2 there is one noticeable DHB that has a larger number of missing values for gestation. This is the Hawke's Bay DHB with 27\% (538) missing values for gestation are missing. Capital \& Coast have the next largest proportion of missing values for gestation $(3.25 \%)$. There are a total of 1,009 missing values for gestation period and hence not ignorable because a large number of missing values come from one DHB (see table 7.2).

Gestation period is defined as the period in weeks that the mother carries the baby before giving birth. This can range from 0-42 weeks, the normal range of gestation is $37-42$ weeks. In this data gestation period ranges from 14-45, with a large number of observations from 37-40 weeks (see figure 5.7). The median is 39 and lower quartile 37 and upper quartile 39. This would be a reasonable range 37-39, to impute for the missing values. This data is continuous.

There are 448 missing values for birth weight. Birth weight is recorded when the baby is first born and is measured in grams. $3.28 \%$ (115) of missing values for birth weight come from Capital \& Coast DHB. 2.87\% of missing values come from Hawke's Bay DHB and 1.43\% from Waikato 
DHB. Since this is not a random pattern of missingess, as such a large proportion come from a few DHBs.

The first investigation for mothers age, ethnic group and deprivation index shows the variables have missing values (gestation, parity and birth weight) all have similar distributions. The second investigation for gestation, parity and birth weight against PPH and DHB shows the pattern of missingness is not random. Some DHBs have a larger number of missing values than others. This illustrates the pattern of missingness is not ignorable and is therefore essential these variables gestation, parity and birth weight are imputed.

In order to prepare the data for imputation, it is important to consider the distributional assumptions of the method that will be used and the selection of variables that are imputed together (Arnold and Kronmal, 2003). As a multiple regression imputation method will be used it is essential to identify the covariates that will be used and to consider the model assumptions. It is also necessary to have consistency with the regression model used for each variable gestation, parity and birth weight. If related variables are not imputed together and then later utilised for analysis this will affect the relationship among them, by that the newly imputed variables will now not be related (Arnold and Kronmal, 2003).

\subsubsection{Imputation Method}

\section{Set up of Data}

Variables used in the regression are required to be formatted to numeric and binary form. This is necessary to allow the regression model to run to calculate the predictors and coefficients of the model. Below is a detailed step of which variables required transformation before the imputa- 
tion function was run.

- Deprivation Index has been formatted into numeric

- Hospital Type is recorded as values 1 and 2 and this is changed to a binary variable which takes the values 0 and 1 .

- Caesarean Section is formatted as binary $(0,1)$ where 1 denotes for an event, that the mother has had a Caesarean section.

- Gender of the baby is in character form M and F and is formatted into binary 0 and 1 where Male $=1$ and Female $=0$.

- As ethnic group is a categorical variable, a set of dummy variables are created to incorporate ethnicity into the model. A binary variable is created for each ethnic group, where 1 is for an event, that the mother is in that ethnic group and 0 is for not in that ethnic group. Five different ethnic variables are created for Māori, Pacific, Asian, Other and NA, where the European ethnic group is the reference group.

- Gestation and DHB are turned into numeric. Birth weight is set to NA if recorded at 9999.

\section{Method}

This method of imputation is based on a similar method as described by Gelman and Hill (2007), Chapter 25.

1. Create a random imputation function in $R$.

Run a simple random imputation, for the three variables that have missing values, based on the observed data. 
A simple random imputation is used to impute missing values of $X$ based on the observed data for these values. This is used first as an interim step. This allows the next steps in the imputation to use all available observations.

As this part does not include information based on other observations it may not make much sense to use this. However it is a convenient starting point to the next step, where all observations are required.

2. Use a multiple regression imputation method that states, if the missing variables are a matrix $Y$ with columns $Y_{1}, \ldots, Y_{k}$ and full observed predictors are $X$, this first imputes all missing $Y$ values using a crude approach (as in the first step) and then imputes $Y_{1}$ given $Y_{2}, \ldots, Y_{k}$ and $X$, then $Y_{2}$ given $Y_{1}, Y_{3}, \ldots, Y_{k}$ and $X$, using the newly imputed values for $Y_{1}$ and continuing this loop until all values are imputed.

3. A function in $\mathrm{R}$ is set up to run a simulation 20 times where a linear model is used for birth weight which is predicted with the variables of parity, mothers age, ethnic groups, plurality, gestation, hospital type, deprivation index, gender, DHB and PPH. The imputed value is obtained by taking the last predicted values from the simulation and is used to impute a value for birth weight where missing.

Gestation also has a linear model and predicted values are used to impute for the missing values of gestation. The same variables are used as in the birth weight model, however gestation has now been substituted with birth weight. 
For parity a GLM with a logit link function is used. The fitted values are used for the prediction of missing values. Again the same variables are used as in the birth weight model, however parity has not been substituted with birth weight.

4. As these models are separate, all variables need to be added back into the full dataset.

Once the imputation method is run the relationships and distributions amongst the variables are explored. The data is scrutinised for outliers and any errors.

\subsubsection{Imputation Results}

Once the imputation has been run, checks need to be made. This will ensure that any imputation that has been made has not greatly altered the distribution of the variables. This section will go through each variable individually to check the distribution and summary statistics prior to and after imputation. Figure 7.3 shows histograms of the variables to be imputed.

\section{Results of Birth Weight Imputation}

Table 7.3 compares the summary statistics of the variable birth weight prior to and after imputation. The minimum birth weight has changed from 225 to 119.2. The median and mean has remained the same. The change in the minimum is expected because when imputing such values the imputation method has no restrictions on the minimum and maximum values. All missing values now have a value which fits well with the distribution of birth weight prior to imputation.

Figure 7.3 shows the distribution of birth weight values of all babies. Prior to imputation the graph shows a normal distribution with a minimum 
weight of $225 \mathrm{~g}$ and maximum weight of $7000 \mathrm{~g}$. On average most new born babies weigh in the range of 3500-4000g, here the birth weight mean is $3466 \mathrm{~g}$ which is just a little lower than average. After imputation the distribution is similar, with the range is from $119.2 \mathrm{~g}$ to $7000 \mathrm{~g}$. Looking at the imputed values there are larger proportions of imputed values in the range of 3000-4500g. This suggest this imputation is a satisfactory approach to imputing birth weight as we would expect a large number to be imputed around this range. The linear regression model gives an Adjusted R-squared of 0.3897 which means the explained variance from this regression is only $39 \%$ of the total variance. (See Appendix B.3)

Table 7.3: Summary of Birth Weight Imputation

\begin{tabular}{lccccccr} 
& Min & 1st Quarter & Median & Mean & 3rd Quarter & Max & NA \\
\hline Prior Imputation & 225 & 3140 & 3490 & 3466 & 3830 & 7000 & 448 \\
After Imputation & 119.2 & 3140 & 3490 & 3466 & 3830 & 7000 & 0
\end{tabular}

\section{Results of Gestation Imputation}

Table 7.4 compares the summary statistics for gestation prior to and after imputation. There is no change to any of the statistics when all values are imputed for. All NA's now have a value after imputation. Figure 7.3 shows prior to and after imputation the distribution of gestation is relatively similar. The linear regression model gives an Adjusted R-squared of 0.3858 , that is only $38.5 \%$ of the variation is explained in this model, and a $p$-value $<0.0001$. (See Appendix B.1).

Table 7.4: Summary of Gestation Imputation

\begin{tabular}{lccccccr} 
& Min & 1st Quarter & Median & Mean & 3rd Quarter & Max & NA \\
\hline Prior Imputation & 14 & 38 & 40 & 39.20 & 40 & 45 & 1,009 \\
After Imputation & 14 & 38 & 40 & 39.20 & 40 & 45 & 0
\end{tabular}

\section{Results of Parity Imputation}

For parity prior to imputation there are more mothers who have not given 
birth before. After imputation this still remains true. Note that this variable is recorded as 0 or 1 where, 0 means the mother has not given birth before and 1 that the mother has given birth at least once before. Table 7.5 shows the summary statistics of parity prior to and after imputation. The minimum and maximum values remain the same as expected and the mean only changes slightly from 0.3600 to 0.3611 (see figure 7.3). To assess the measure of fit the binomial logistic regression model gives AIC of 31,233 and Residual deviance of 31,165 on 29,462 degrees of freedom (See Appendix B.2).

Table 7.5: Summary of Parity Imputation

\begin{tabular}{lccccccr} 
& Min & 1st Quarter & Median & Mean & 3rd Quarter & Max & NA \\
\hline Prior Imputation & 0 & 0 & 1 & 0.3600 & 1 & 1 & 26,083 \\
After Imputation & 0 & 0 & 1 & 0.3611 & 1 & 1 & 0
\end{tabular}

\subsubsection{Summary}

Using an multiple imputation seems to be a reasonable approach to this data. It does not influence the variables imputed in anyway. It is important for these variables to be imputed as they are potential predictors for $\mathrm{PPH}$. It is also essential to choose the most appropriate imputation method. If this is not done then this could severely impact the results and analysis.

When identifying and investigating the variables of interest for imputing, it is important to examine the distribution of missingness of the variables (Rubin, 1976; Scheffer, 2002). In particular exploring what is missing, how much is missing and the type and pattern of missingness (e.g. MCAR, MAR, not ignorable). If it is non-ignorable then imputation methods will need to be selected and used to fill in the missing data. If left missing, the data can cause bias and lead to incorrect inferences from our conclusions. 

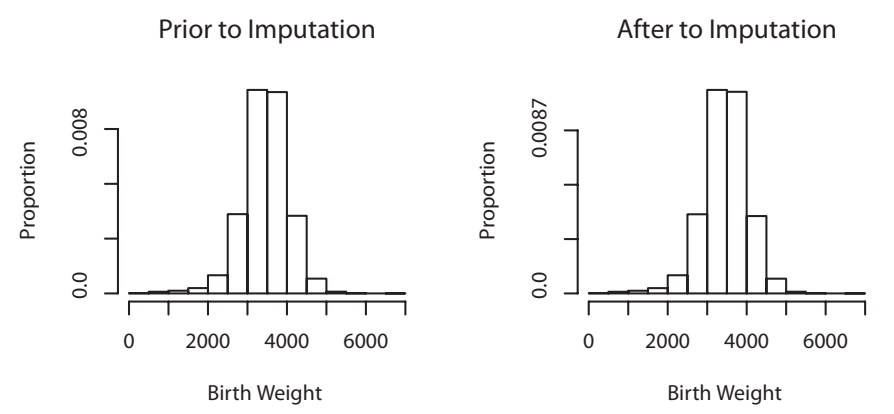

Distribution of Imputated Values
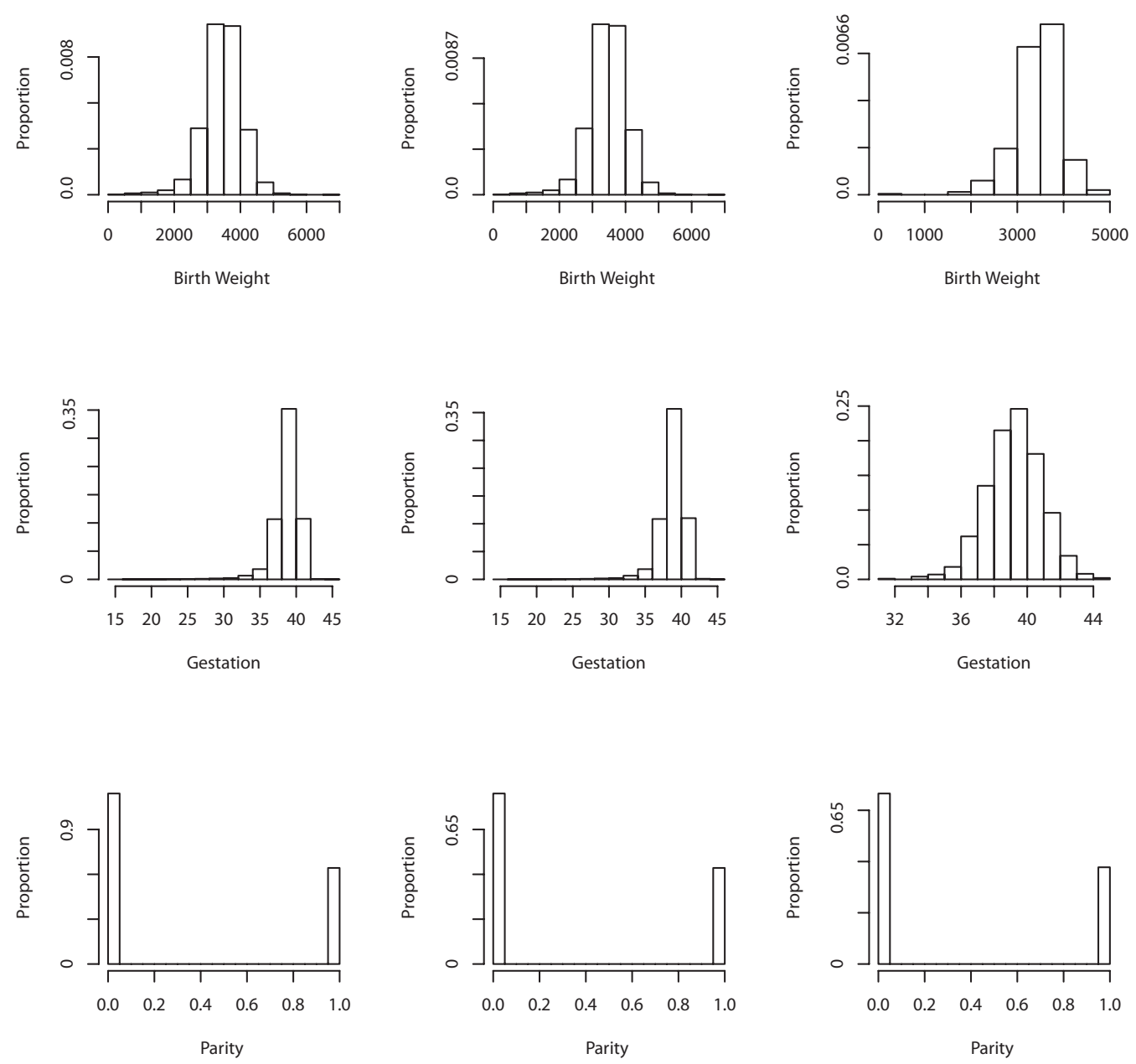

Figure 7.3: Distribution of birth weight, gestation and parity, before and after imputation

An imputation method needs to be carefully chosen depending on the characteristics of the data. Here gestation and birth weight are treated as continuous variables and parity is binary variable. These characteristics need to be taken into account.

A Multiple regression approach was chosen as this would give the most appropriate results. They would be unbiased, specific to the variables of 
choice and produce reliable values. This imputation approach was carried out simultaneously for all three variables, to make sure the relationships among variables are not ignored. If they were ignored then the final dataset would be misleading and these relationships ignored.

The imputation process involved detailed explorations of the data in order to select which variables to impute. This involved investigating which variables should be imputed together, distributional assumptions and identification of outliers and extreme values.

Looking at complete case distributions and imputed values showed distributions are similar for gestation, parity and birth weight. Different distributional analysis were considered for gestation, results found a general linear model was preferred over a Poisson model. Many imputation methods were considered, however multiple random regression was the preferred approach.

\subsection{Logistic Regression Results}

\section{Applying the Logistic Model to the data}

The aim for this regression is to assess the probability of PPH occurring based on explanatory variables and their relationships (for example birth weight, gestation, ethnicity etc). As there are a large number of potential predictors to choose from, it is important to identify those that are useful. The first step in the risk adjusted funnel plot is to identify the important variables significant to the outcome of PPH. Once we identify these variables adjustments can be made by using the predicted probabilities from the model.

In a ideal world we would want to include every explanatory variable 
available that may be useful in predicting the response, however this task would be too onerous and would be very difficult to interpret. It is preferable to use a selection of steps to make the model as simple as possible and therefore easier to interpret. Choosing the appropriate model for our data to fully explains the outcome $\mathrm{PPH}$, requires iterations of logistic regression to help identify the 'important' variables. A selection of backwards elimination, forward selection and stepwise regression model methods are used to determine the best model.

Following consultation with an expert in this area (Sibanda T, personal communication) and literature (Waitemata District Health Board, 2006; University of Virginia Health System, 2010; Jacobs, 2012; Combs et al., 1991) it was determined that the variable and interactions in figure 7.4 (the gray shaded areas) are the most important to investigate. These variables and their interactions are chosen in the logistic regression method, to help choose the most appropriate model for the risk adjustment method. This leaves us with a choice of 13 main effects and 36 possible interactions to look at.

Once the stepwise regression has run, a logistic regression is run using the variables and their interaction identified in the stepwise method. This is to obtain the predicted probabilities and apply these to the risk adjusted method.

\section{Method}

To first identify the important factors the following procedure is used to fit the model using proc logistic in SAS.

In all models the response variable is $\mathrm{PPH}$. The predictors include the main effects and some two way interactions. Figure 7.4 shows the main effects and interactions that could be potential predictors of $\mathrm{PPH}$, these 


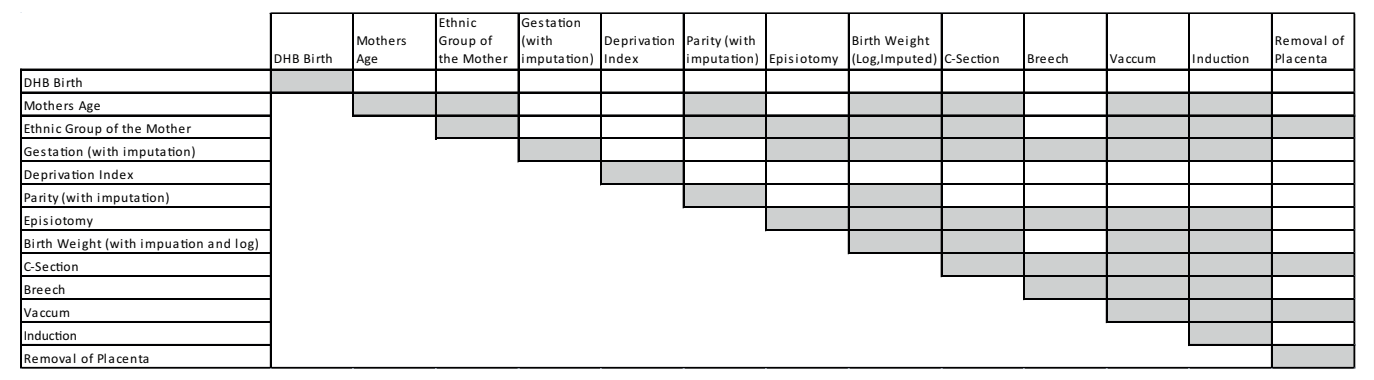

Figure 7.4: Matrix of Regression Variables for selection

are shaded in gray. I used significant level 0.05 (5\%) to identify the variables that are to remain or be removed in the regression model. Any variable that had a p-value of 0.05 or greater were eliminated from the model. PPH=1 is defined as an event has occurred. Even though a stepwise logistic regression method is the preferred option, both forward and backwards elimination were run to be used as a comparison against the stepwise method. This was to see if both or one of these methods produced the same results. The forward selection produced the same results as the stepwise (see Appendix C). The backwards elimination method added some extra factors including mothers age and the interaction between mothers age ethnic group, mothers age and birth weight, mothers age and C-section.

The stepwise method is the chosen method for the choice of variables to predict PPH. It shows 10 main effects and 9 interactions are significant factors in predicting PPH. Figure 7.5 shows the matrix of the variables considered potential factors, in gray and those in blue that have been chosen (see Appendix C.1).

The goodness of fit for the stepwise regression for selection of model shows the R-Square is 0.0538 which shows this model only explains $5 \%$ of the variation. The Hosmer and Lemeshow Goodness of Fit Test indicates the quality of model fit $\mathrm{p}=$ value $=0.0023$ 
The next model is the final model to use for the risk adjusted funnel plot. This takes the variables and their interactions as identified in the stepwise regression as significant. This model is used to obtain the predicted probabilities to apply to the risk adjusted funnel plot.

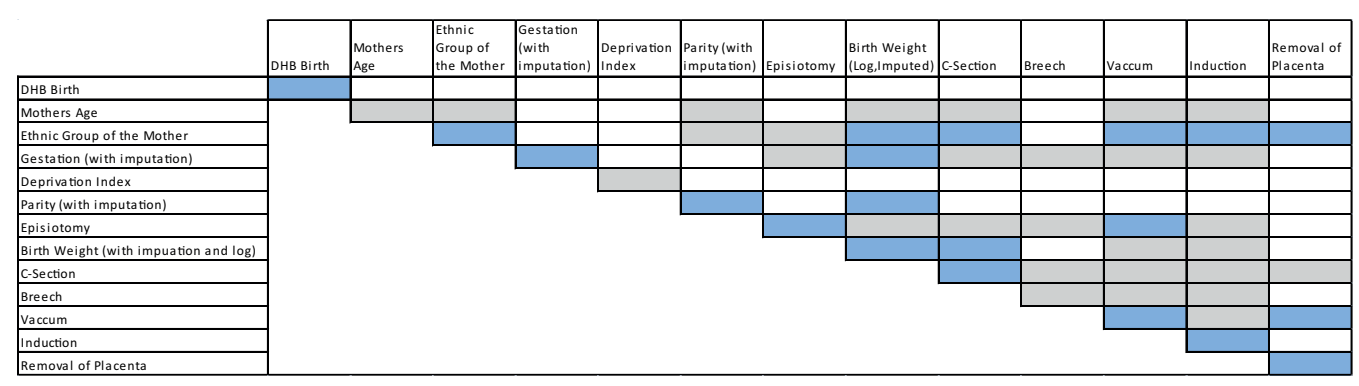

Figure 7.5: Matrix of Regression Variables for that are chosen

Figure 7.6 displays the log odds ratio and the $95 \%$ confidence interval for each factor. Asian and Pacific ethnic groups compared to European have a lower risk of PPH if all other factors are held constant. Ethnic groups Māori and Other have a higher risk for PPH compared to the European group, if all other factors are held constant. An increase in the baby's birth weight has a decrease in the risk of $\mathrm{PPH}$, if all other factors remain constant. Parity and C-section have a increased risk for PPH, where all other factors are held constant.

\subsection{Risk Adjusted Funnel Plots}

The following method describes how the risk adjusted funnel plot is constructed.

1. Run a logistic regression using PPH as the event where the probability that PPH has occurred, event occurred $=1$, where $P(p p h=1)$ 


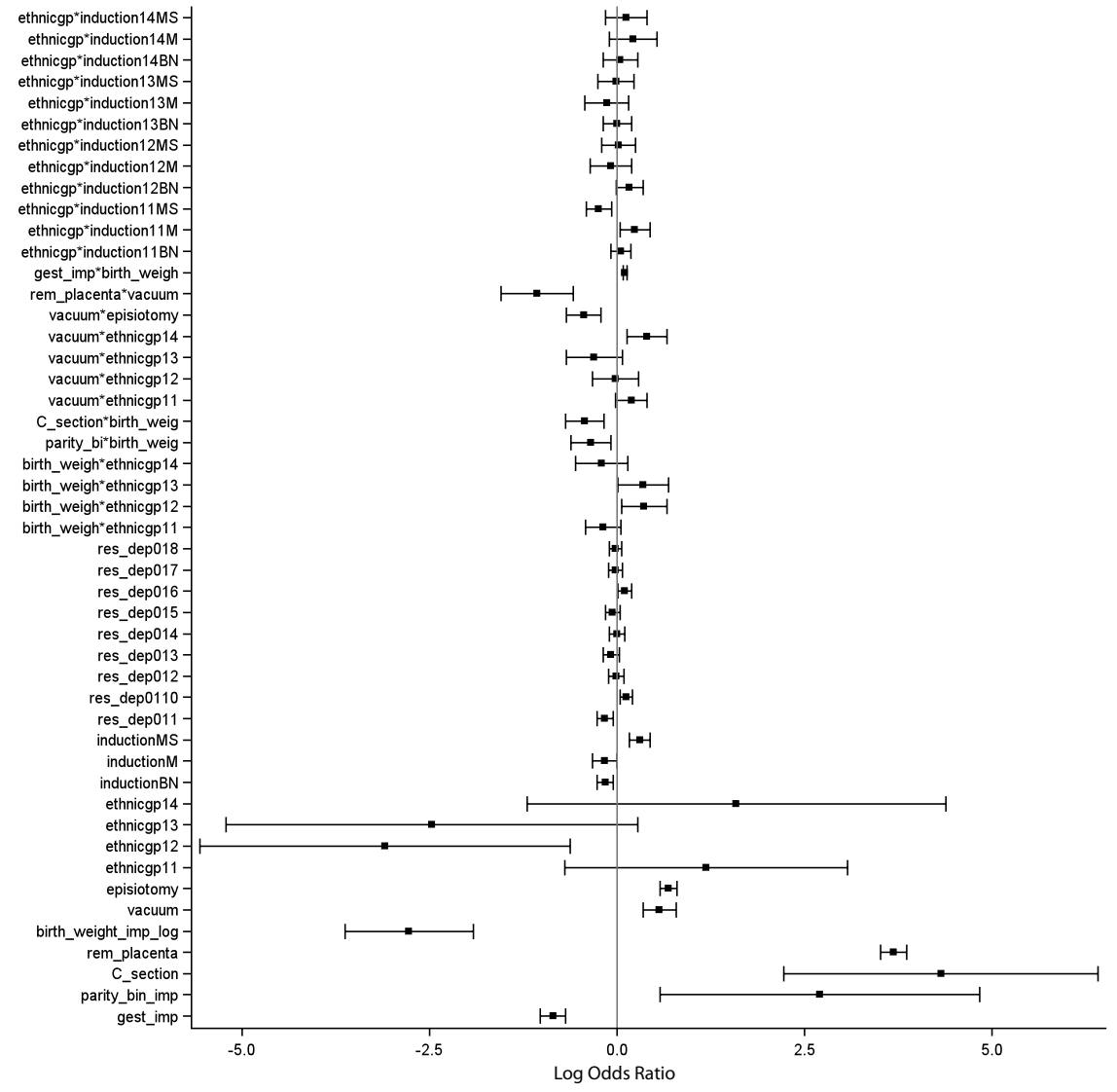

Figure 7.6: Log Odds Ratio

The covariates chosen here are from the previous stepwise logistic regression in section 7.2. This will obtain the predicted values for each individual mother.

2. Calculate the sum of predicted values for each DHB $i$

This is the expected number of PPH, $E_{i}=\sum j \hat{y}_{i j}$ for each $i$ where,

$E_{i}=\sum \hat{p}_{i} j$, for each $i \ldots \hat{p}_{i} j$ is the predicted probability of PPH for 
mother $j$ in the DHB $i$.

3. Calculate the risk adjusted rate $y_{i}$

Risk Adjusted rate $=\frac{O_{i}}{E_{i}} \times \hat{\theta}$

where

$\hat{\theta}_{O}$ is the national rate of $\mathrm{PPH}$.

$O_{i}$ is the number of mothers with PPH in DHB $i$

$E_{i}$ is the expected number of PPH for DHB $i$

Therefore the risk adjusted rate is

$$
=\frac{O_{i}}{\sum \hat{p}_{i j}} \times \hat{\theta}_{0}
$$

$\hat{p}_{i j}$ is the predicted values for each mother $j$ in DHB $i$

4. To plot the adjusted funnel plot. Plot $Y_{i}$ against the precision parameter $n_{i}$, with the target $\theta_{0}$ shown as a horizontal line and superimpose the control limits on the plot as function of the parameter $n_{i}$.

\subsubsection{Risk Adjusted Funnel Plot Results}

After adjusting for variables as described in section 7.2, figure 7.7 shows the adjusted funnel plot and its control limits. From a quick glance there are many DHBs that still remain outside the control limits. There are now 10 DHBs that are out of control, compared to figure 6.6 there were $7 \mathrm{DHB}^{\prime} \mathrm{s}$ that sit outside the ctonrol limits. Ten of the 21 DHBs lie outside the $99.8 \%$ control limits, with one of these on the cusp of the limits. Eight DHBs lie with the 95\% control limits. From the adjusted funnel plot (figure 7.7) it is very difficult to draw any conclusions because many DHBs are divergent from one another. Over half of the DHBs fall outside the $95 \%$ control limits. 


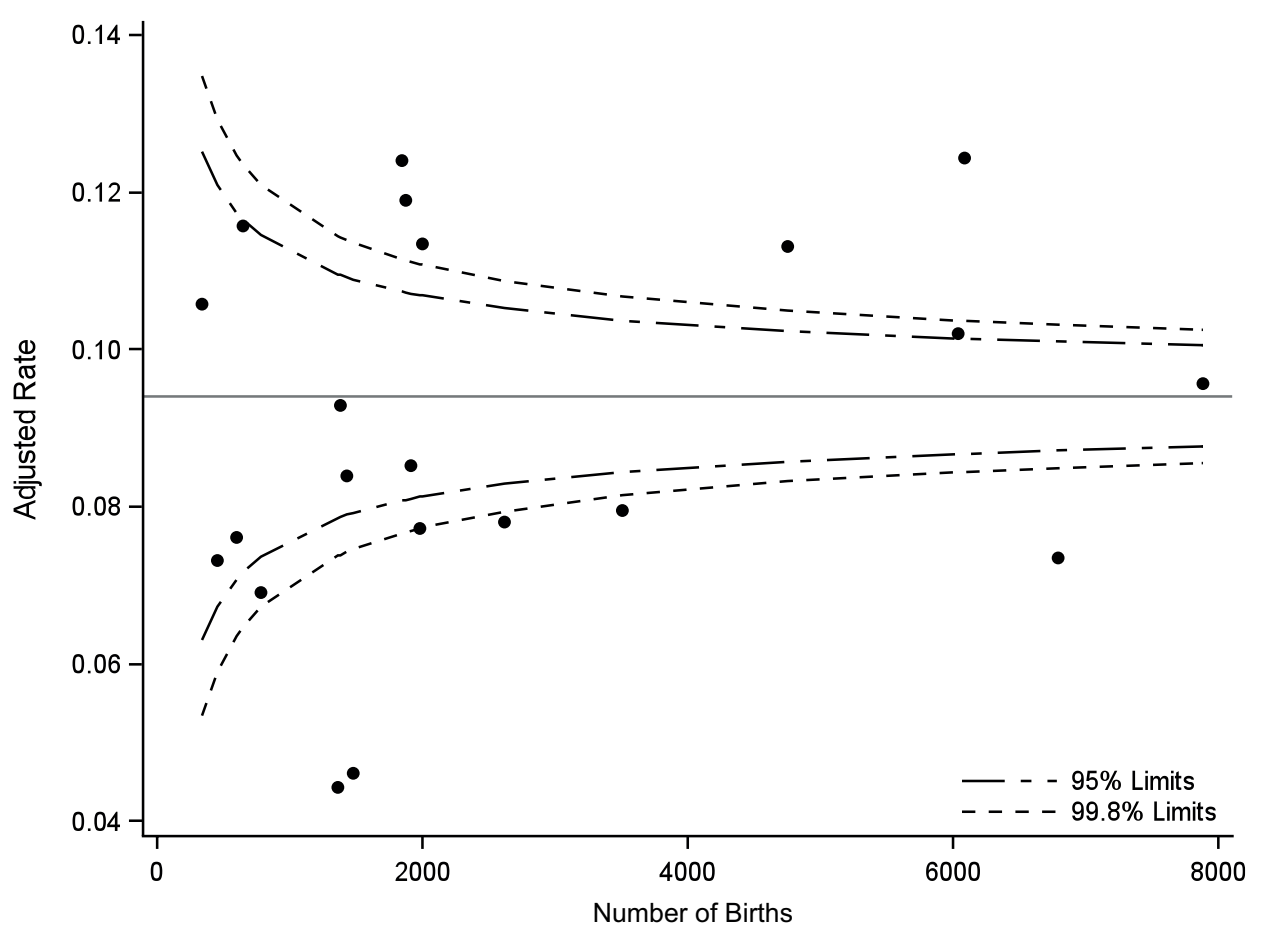

Figure 7.7: Adjusted Rate

Looking at figure 7.7 in more detail, some DHBs that have shifted more considerably than others compared to the unadjusted funnel plot. In figure 6.6 Auckland DHB was at the point on the top right of the figure, the most extreme outlier (with an unadjusted rate of 0.140 ). In the adjusted funnel plot (see figure 7.7) Auckland maintains the same position at the top right of the funnel plot but now has moved closer towards the control limits (with a rate of 0.125). Nelson Marlborough and Southland DHBs still remain well outside the control limits. Nelson Marlborough and Southland are the two points close to each other at the bottom left of the graph. Their rates have moved from $0.043,0.038$ to $0.045,0.043$ respectively. Waitemata is the point at the right of the graph outside the lower control limits. After adjustment Waitemata still remains well outside the control limits. Northland, Otago, MidCentral and Waikato have 
now moved from being inside the confidence limits to outside the confidence limits after the adjustment has been made. These DHBs are above the upper confidence limits. Bay of Plenty has moved inside the $99.8 \%$ confidence limits. Capital \& Coast have moved just outside the lower $99.8 \%$ confidence limit. In the unadjusted funnel plot Capital \& Coast were on the cusp of the $99.8 \%$ confidence limit.

This shows us that some DHBs have moved outside the control limits and some inside the limits. These DHBs rates have moved closer to the natural rate as expected (Counties Manukau and Lakes District).

Using and adjustment method the variables available, it is noticeable that some DHBs move closer to mean and some move further away. Suggesting there are still population differences between the DHBs and furthermore there are other factors that are not measured and availble for us to use, that may need to be considered when adjusting for PPH. This model shows larger variability between DHBs as many DHBs fall outside the $95 \%$ and $99.8 \%$ control limits, indicating overdispersion around the target. This can occur because of unmeasured covariates not available or have not been measured or recorded. It may be more appropriate to use a Winsorisation approach to adjust for the overdipsersion.

\subsection{Winsorised Funnel Plots}

\section{Winsorised Estimate}

An approach for this over-dispersion would be to use a standard GLM approach. This introduces a factor $\phi$ that will inflate the null variance by a certain percentage. This is a multiplicative variance model where each DHB's variance will change by a different amount, but through a common $\phi$. It is expected that DHBs with larger variances will be affected more. 


\section{Method}

1. Calculate the naive Z-scores for each DHB

$$
Z_{i}=\frac{Y_{i}-\theta_{o}}{\sigma}
$$

where

$p_{i}=$ unadjusted rate of PPH for each DHB

$\theta_{0}=\mathrm{NZ}$ national rate of $\mathrm{PPH}$

$\sigma=\sqrt{\left(\theta_{o}\left(1-\theta_{o}\right) / n_{i}\right)}$

where $n_{i}=$ sample size of each DHB

2. Rank these Z-scores from largest to smallest

3. Identify $Z_{q}$ and $Z_{1-q}$ the $100_{q}$ percentiles, the largest and lowest naive $Z$-scores, where $q=0.1$. Take the top and bottom $10 \%$ of the $Z_{i}$ DHBs. This would be $21 \times 10 \%=2.1$. Identify the bottom two and top two DHBs. Take the second highest Z-score and assign this to the top two DHBs Z-scores. For the lowest two DHB Z-scores take the second to lowest $Z$-score and assign this to the two lowest DHB Z-scores. Leave the rest of the Z-scores unchanged. These are newly calculated Zscores, $Z^{w}$ that have been pulled in by the 'winsorised' $10 \%$. From this, new confidence limits can be calculated.

4. Calculate the estimate $\hat{\phi}$ in (4.4) using $Z^{w}$.

$$
\hat{\phi}^{W}=\frac{1}{I} \sum_{i} Z_{i}^{W}(q)^{2}
$$

where $I=$ the total number of DHBs.

$\sum_{i} Z_{i}^{W}(q)^{2}=$ is the sum of the Z-scores new, squared.

The estimate $\hat{\phi}^{W}$ can also be multiplied by a debiasing factor $w(q)$ if all DHBs are in control and the only variability is due to overdispersion. If this is the case, the variance of the winsorised Z-statistics 
will tend to be less than $\phi$. If it is assumed that $Z^{W}(q)$ is from a qWinsorised standard normal distribution, then the variance can be calculated as in ?? paper:

$$
\mathbb{V}\left[Z^{W}(q)\right]=1+2 q\left(z_{q}^{2}-1\right)-2 z_{q} e^{-z_{q}^{2} / 2} / \sqrt{2 \pi}=1 / w(q)
$$

so $\hat{\phi}=w(q) \hat{\phi}^{W}$, where $z_{q}=\Phi^{-1}(1-q)$.

where $\Phi^{-1}(1-q)$ is the $100(1-q) \%$ percentile of the standard normal distribution.

So if we are to use a winsorised estimate of $10 \%$ then $w(0.10)=1.47$ and the variance would then be $\mathbb{V}\left[Z^{W}(q)\right]=1 / 1.47=0.68$. This will underestimate the variance by $32 \%$.

5. The boundaries can then be calculated by

$$
\theta_{0} \pm z_{p} \sqrt{\hat{\phi} g\left(\theta_{0}\right) / n_{i}}
$$

where

$\theta_{0}=$ Target NZ rate $\hat{p}$

$Z_{p}=95 \%$ confidence interval limits at 1.96

$\hat{\phi}=$ winsorised estimate

$g\left(\theta_{0}\right)=\theta_{0}\left(1-\theta_{0}\right)$

$n_{i}=$ precision parameter $=$ sample size

If the data has no true over-dispersion, then we can check for this. It can be assumed that $I \hat{\phi}$ has approximately a $\chi_{I}^{2}$ distribution, which means the expected value $\mathbb{E}(\hat{\phi})=1 / I$, and that the $\mathbb{V}(\hat{\phi})=2 / I$. As the above is an assumption, one must test this assumption before applying this to the data. There are two points to check: (Spiegelhalter, 2005a) 
1. That if $\hat{\phi}<1+2 \sqrt{2 / I}$ assume that $\phi=1$;

2. If the estimated is $\hat{\phi}>1+2 \sqrt{2} / I$ assume there is overdispersion.

Here the estimate $\hat{\phi}$ is 10.85 and $1+2 \sqrt{2} / I=1.13$ where $I=21$. Therefore $10.85>1.61$ and $\hat{\phi}$ is not less than 1 . This concludes there is overdispersion and adjustments can be made for overdispersion.

\subsubsection{Winsorised Estimates with Random Effects}

The other approach for adjusting for over-dispersion is using the winsorised estimate as calculated above and adding a random effects term.

In this approach we assume that each DHB has its own distribution for the PPH rate, that is $\mathbb{E}\left(p_{i}\right)=\theta_{i}$ and $\mathbb{V}\left(Y_{i}\right)=s_{i}^{2}$ (Spiegelhalter, 2005a). For DHBs whose rates are on target $\theta_{i}$ is distributed with mean $\theta_{0}$ and standard deviation $\tau$. The null hypothesis will therefore be a distribution, (for example $\left.\theta_{i} \sim N\left(\theta_{0}, \tau\right)\right)$ rather than a point $\left(\theta_{i}=\theta_{0}\right)$. The standard deviation $\tau$ can be estimated using the methods of moments estimator.

Method of moments estimate

$$
\hat{\tau}^{2}=\frac{I \hat{\phi}-(I-1)}{\sum_{i} w_{i}-\sum_{i} w_{i}^{2} / \sum_{i} w_{i}}
$$

where $w_{i}=1 / s_{i}^{2}$

$s_{i}^{2}=$ is the variance for each DHB proportion $p_{i}\left(1-p_{i}\right) / n_{i}$

$\hat{\phi}$ is the test for heterogeneity if $\hat{\phi}<(I-1) / I$ then $\tau$ is to be set to 0 and complete homogeneity is assumed. 
The funnel plot boundaries are given by

$$
\theta_{0} \pm z_{p} \sqrt{\mathbb{V}\left(Y \mid \theta_{0}, n_{i}\right)+\hat{\tau}^{2}}
$$

\section{Method}

To apply this to the data the following steps below are used.

1. Calculate $\hat{\tau}^{2}$ as in equation 7.6

where

$\hat{\phi}=$ winsorised estimate

$I=$ number of DHBs

2. Calculate the control limits

$$
\theta_{0} \pm z_{p} \sqrt{\mathbb{V}\left(Y \mid \theta_{0}, n_{i}\right)+\tau^{2}}
$$

where

$\theta_{0}=$ Target of NZ Rate $p$

$Z_{p}=95 \%$ confidence limits

$V\left(Y \mid \theta_{0}, n_{i}\right)=\frac{g\left(\theta_{0}\right)}{n_{i}}=\frac{\theta_{0}(1-\theta)}{n_{i}}$

where,

$n_{i}=$ precision parameter

$\hat{\tau}^{2}$ can be calculated as

$$
\hat{\tau}^{2}=\frac{I \hat{\phi}-(I-1)}{\sum_{i} w_{i}-\sum_{i} w_{i}^{2} / \sum_{i} w_{i}}
$$

where,

$I=$ number of DHBs

$\hat{\phi}=$ winsorised estimate

$w_{i}=1 / s_{i}^{2}$

$\operatorname{Var}\left(p_{i}\right)=s_{i}^{2}$ variance of proportion of each DHB $p_{i}\left(1-p_{i}\right) / n_{i}$

$p_{i}=$ unadjusted rate of PPH for each DHB $i$. 


\subsubsection{Winsorisation Funnel Plot Results}

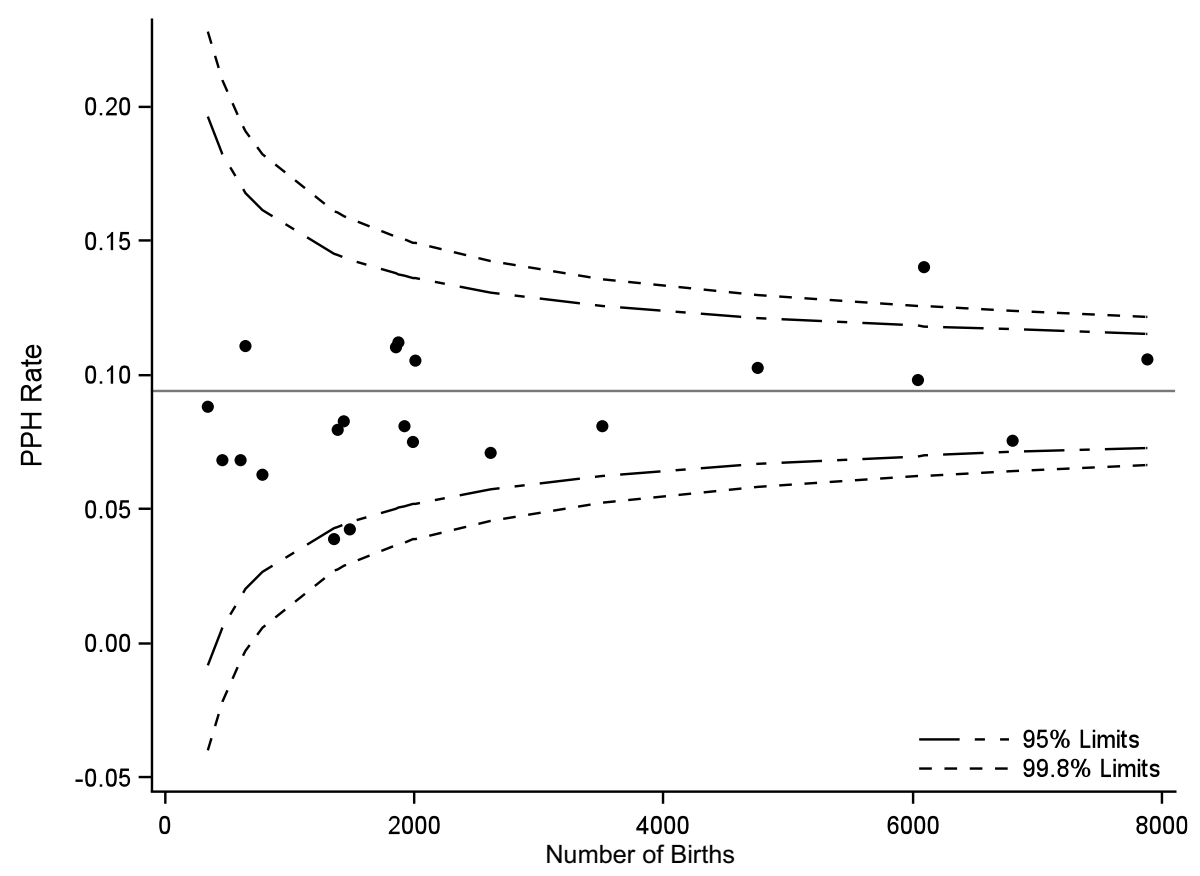

Figure 7.8: Winsorisation Funnel Plot

When estimating the inflation factor usin gresiduals subject to $10 \%$ winsorisation, the winsorised estimate, 19 DHBs in figure 7.8 appear to be well within the $95 \%$ control limits. Two DHBs are on the cusp of the $95 \%$ control limits at the population mark about 1600. These are Southland and Nelson Marlborough. One DHB is outside the $99.8 \%$ control limits, at the population mark of approximately 6000. This is Auckland DHB. This has moved closer to the $99.8 \%$ limits where as in the previous funnel plots Auckland was situated well outside the limits. With the use of the $10 \%$ winsorised estimate has caused the control limits to widen, causing nearly all DHBs to be inside these limits. 
When examining the previous two figures 6.6 and 7.7 these funnel plots have identified a few outliers. One in particular was very extreme compared the rest. This is Auckland DHB. As this was such an extreme outlier this one DHB could cause the results to shrink and conceal the true meaning of the funnel plot.

Sometimes when there are particular large estimates for a DHB it can cause an increase in the estimate $\phi$, which can widen the funnel limits and make it very difficult to determine cases that are of interest. A more robust approach could be to use an random effects term in the winsorisation. And secondly remove Auckland DHB and their observations from the data and rerun the analysis. To see if this makes any difference to the divergence among DHBs (Marshall et al., 2004).

\subsubsection{Winsorisation Random Effects Funnel Plot Results}

The adjustment of adding a constant random effects term to the model, makes a large difference. The confidence limits are narrowed compared to the winsoriation funnel plot (see figure 7.8). There are a large number of DHBs outside the confidence limits. $\hat{\tau}^{2}$ is 0.00032 , a very small value and hence why this funnel plots looks very similar to the unadjusted funnel plot, the random effects adjustment is making almost no difference.

Figure 7.9 shows the funnel plot of the winsoristed estimates using the random effects approach. This shows the PPH rate with a constant term added against the DHB population. Of the $21 \mathrm{DHBs}, 8 \mathrm{DHB}$ fall outside the $99.8 \%$ control limits and 13 DHBs fall outside the $95 \%$ confidence limits. Auckland DHB is the extreme outlier, the point at the top right of the graph. Other DHBs who were outliers in the unadjusted plot remain out- 


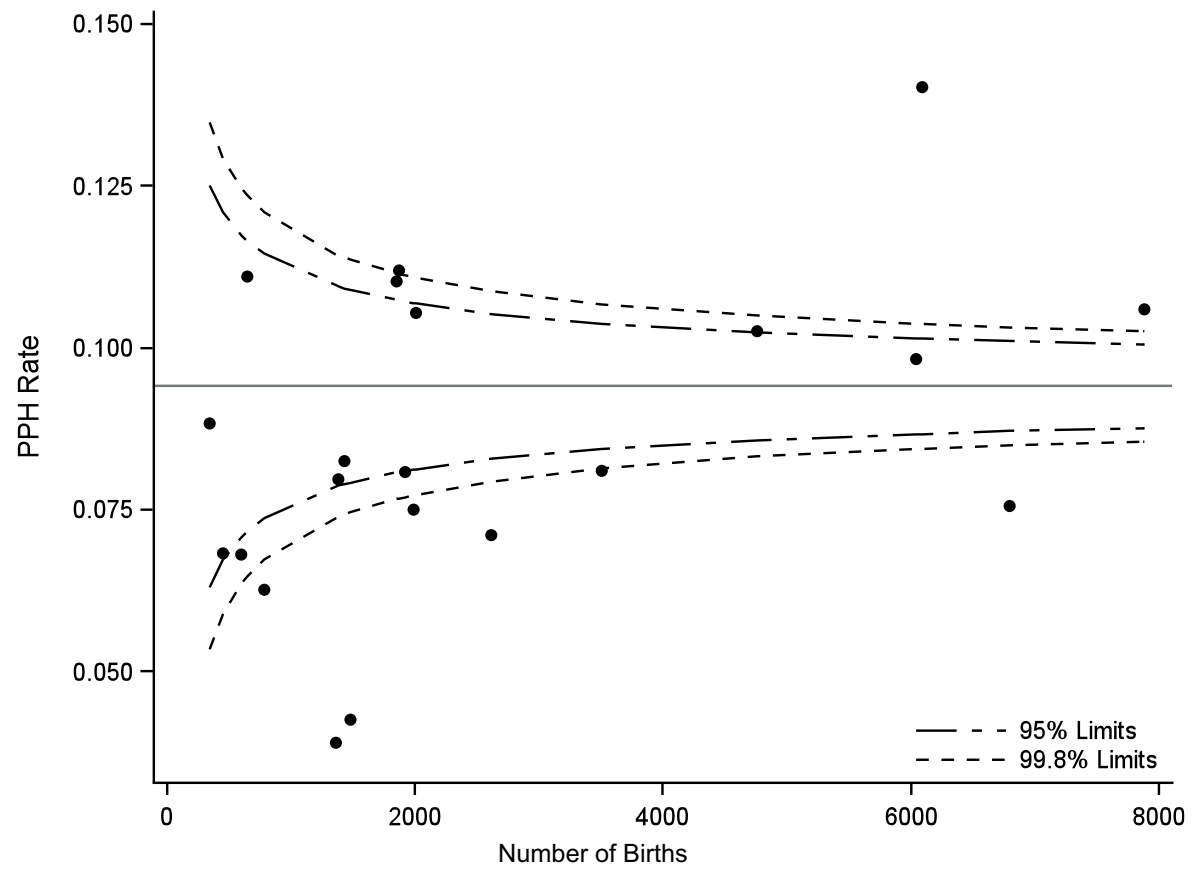

Figure 7.9: Winsorisation Random Effects Funnel Plot

liers in this funnel plot to.

As Auckland DHB seems to be an extreme outlier and could potentially be influencing the results the next step would be to remove Auckland DHB and rerun the analysis, to see if this makes any difference to the control limits.

\subsubsection{Winsorisation Results Excluding Auckland DHB}

As identified in figures (see 7.7, 7.8, 7.9) there was one extreme outlier observed, Auckland DHB. Auckland DHB has the highest rate of PPH of $14 \%$.

Figure 7.10 is a funnel plot using the winsorisation method excluding 


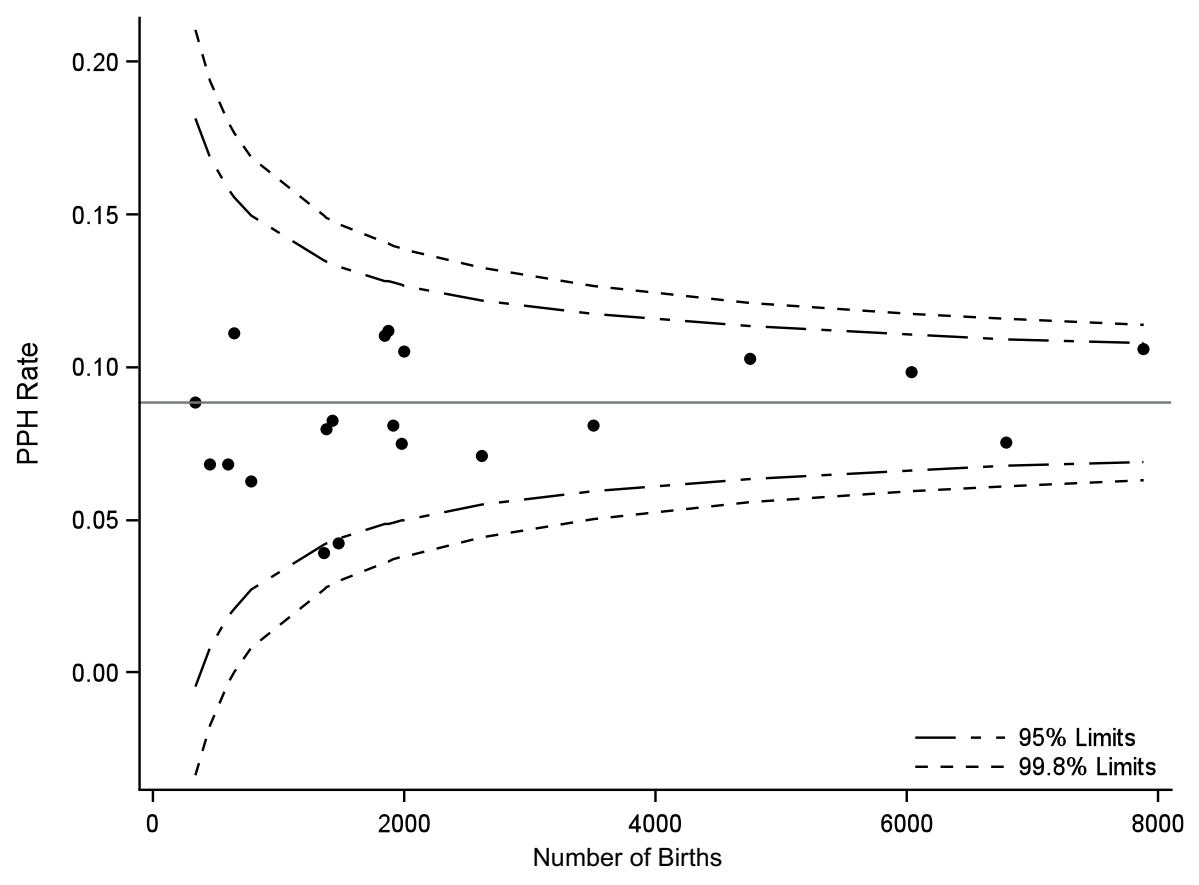

Figure 7.10: Winsorisation Funnel Plot Excluding Auckland DHB

Auckland DHB and their observations. A new national PPH rate $(p)$ is calculated. This is now $8.8 \%$. Using the same winsorisation method as in section 7.4, equation 7.5 is used to calculate a new set of control limits. $p_{i}$ is plotted against the precision parameter $n_{i}$, with the target $\theta_{0}$ as the horizontal line and the control limits superimposed on the plot as a function of the parameter $n_{i}$.

Figure 7.10 uses the standard approach excluding Auckland DHB. There are 2 DHBs that lie outside the $95 \%$ confidence limits and one on the cusp. All other DHBs are situated inside the control limits. The two DHBs that sit outside the 95\% confidence limits are Southland and Nelson Marlborough with rates of 0.0389 and 0.0425 respectively. The other DHB that sits on the cusp of the $95 \%$ confidence limit is situated to the far left of the graph and this is Counties Manukau DHB at a rate of 0.1058 . 
Compare this to figure 7.8 which is the winsorisation approach inclusive of Auckland. Excluding Auckland has not made much difference to the funnel plot results.

\subsubsection{Winsorisation with Random Effects Results Exclud- ing Auckland DHB}

This section uses the winsorisation with random effects method as described in section 7.4.1. The only difference here is that Auckland DHB and their observations are removed from the dataset. A new method of moments estimate is calculated using equation 7.6 and new funnel plot boundaries are also calculated using equation 7.8. The PPH rate is then plotted against each DHBs population with the $95 \%$ and $99.8 \%$ control limits superimposed on the plot.

Figure 7.11 shows the funnel plot for winsorisation method with a random effects term added, excluding Auckland DHB. The funnel plot limits narrow and many more DHBs sit outside the confidence limits. There are now 13 DHBs that lie outside the 95\% confidence limits and 9 DHBs that lie outside the $99.8 \%$ confidence limits. 


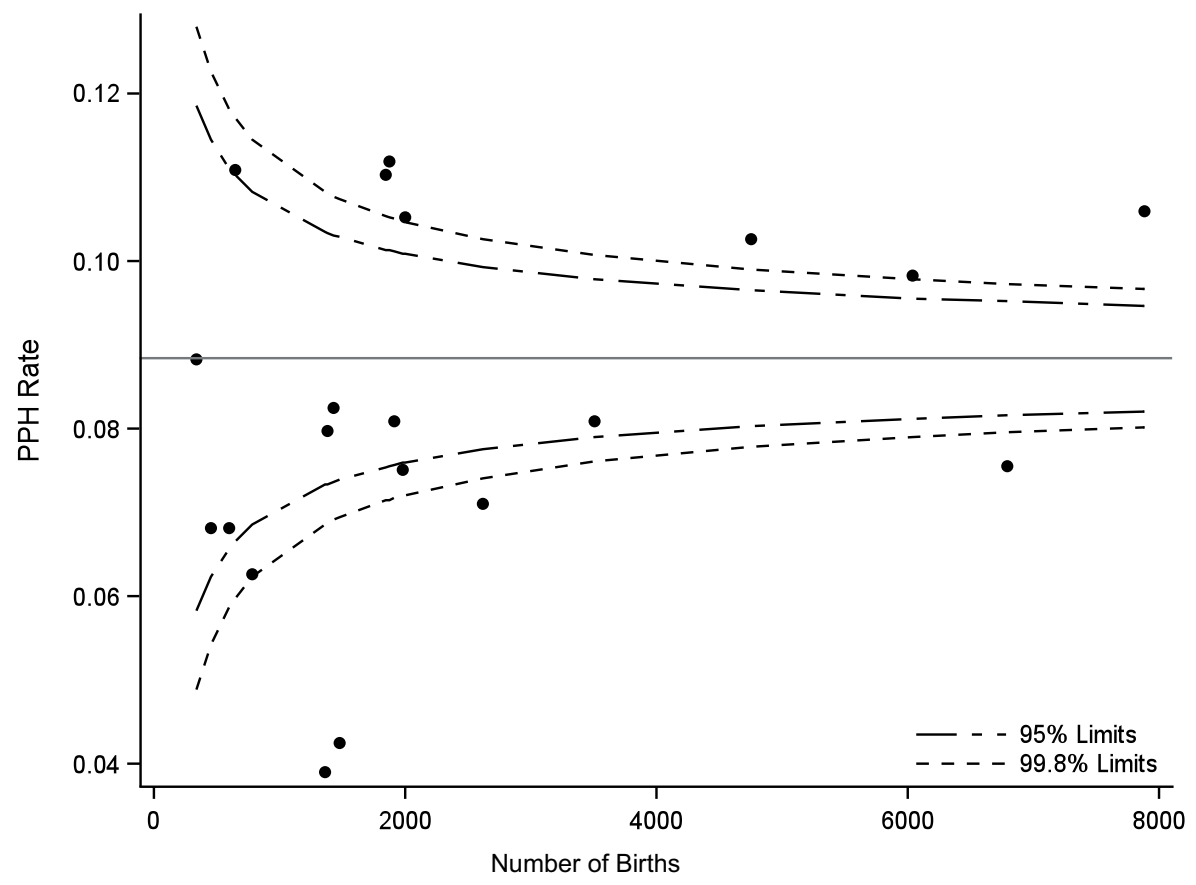

Figure 7.11: Winsorisation Random Effects excluding Auckland DHB 


\section{Chapter 8}

\section{Discussion}

In this thesis the work carried out shows a useful comparison of DHBs performance against PPH rates for the 2007 year in NMDS. The thesis uses a range of methods to demonstrate the use of funnel plots for measuring performance of institutions. This analysis has established a picture of performance across NZ DHBs regions in relation to each other and their PPH rates. The work itself is unique as the focus is on DHBs in NZ in a medical context where previously very little research has been carried out in the subject area the variation in outcomes of mothers and births across DHBs.

In the EDA we found there are differences in PPH among the demographics, maternal and birth characteristics. There are also differences among the DHBs and their characteristics. The rates of PPH are different, the population mixes of DHBs are made up of a varying range of ethnic groups, ages and deprivation indexes. We also found in the EDA there are a lot of factors associated with PPH. This indicates the need for adjustment.

Following the EDA we found some important predictors of PPH had a large number of missing values and that these values were not missing completely at random. These predictors were gestation, parity and birth weight. Several DHBs had more missing data than other DHBs for some 
variables. Imputation was then carried out to impute values for the missing values. A multiple regression imputation approach was used and the results from the imputation process were analysed. The variables imputed for are parity, birth weight and gestation.

Once the imputation had been run, the first funnel plot was constructed. This is the unadjusted funnel plot where the raw rate of $\mathrm{PPH}$ is plotted against each $\mathrm{DHB}$. The unadjusted rate shows large variability among the rates of PPH for each DHB's population. It shows there are some extreme outliers at both ends of the population spectrum. Some DHBs with smaller numbers of births have a low rate of PPH and some DHBs with large rate of PPH. The large variation among the DHBs suggests there is overdispersion among the DHBs consequently factors need to be taken into account for PPH and a risk adjusted funnel plot is constructed.

This risk adjusted funnel plot attempts to take into account factors that are good predictive measures for PPH. A logistic regression model is run to identify these covariates and then an adjustment is made to the $\mathrm{PPH}$ rate. This risk adjusted funnel plot shows some DHBs rates move away and others adjust considerably closer to the target. This suggests there are potentially some factors that are not adjusted for because large overdispersion around the target still remains. Further methods are used to adjust the control limits of the plot.

First a winsorised estimate is calculated which shows shrinkage of the adjusted PPH rate and a widening of the control limits. This is because an multiplicative factor has been added to the control limits and adjustments made. A 10\% winsorised estimate is added which inflates the control limits. It shows the larger DHBs with the higher Z-scores have moved the control limits outwards. It is from this point that you can really identify a few outliers that could be causing the change in the control limits. As 
there are such large estimates for one DHB this can cause an increase in the estimate $\phi$, which can widen the funnel limits and make it difficult to determine cases that are of interest. A more robust approach may be to include a random effects term.

With the addition of the random effects term causes the control limits to narrow. Using a winsorised estimate with a random effects term adds a constant term to the equation and appears to make a large difference to the control limits. The funnel plot, figure 7.9 shows no real difference compared to fiugre 6.6 as there are still a large number of DHBs outside the control limits. However there remains one outlier which could still cause shrinkage of the control limits of the DHB rate around the target.

On four funnel plots displayed one DHB stands out from the rest (see figures 6.6, 7.7, 7.8, 7.9). This is Auckland DHB. This DHB could have a large influence on the control limits produced. It was decided to remove Auckland DHB and its observations from the data and the analysis to be run again for the winsorised and random effects funnel plots.

With the exclusion of Auckland DHB the winsorisation funnel plot shows that nearly all DHBs are now within the 95\% control limits and two DHBS are just outside these limits. This shows that Auckland DHB has a strong influence on this funnel plot. The results from the winsoriation with random effects term added shows variability among DHBs as many DHBs sit outside the $95 \%$ control limits. With this additive model the control limits have now shrunk.

Future work based on this research could involve obtaining more factors associated with PPH. There were a few variables unavailable to predict PPH such measures may include: who the baby was delivered by(obstetrician or midwife), where the baby was delivered (home birth or hospital), the 
mothers previous medical history (previous PPH, C-section etc) these measures could be important to include in future analysis. Other such useful information that was not recorded is the mother's weight, infections and other medical details also could be useful measures of PPH.

Other work could involve an improved measure on the target rate $\theta$. A Bayesian hierarchical method can be used to improve the precision of the target and can control for variation that can occur. This would be an interesting piece of analysis to conduct and to compare the Bayesian method to the methods used in this research.

This analysis will provide useful information to health care providers for funding, quality of care and administrative processes. These set of funnel plots are a useful way to provide a visual analysis of providers against a outcome and can be used in any context. This research is a practical approach for hospitals, providers and their funders to identify areas for more in-depth self monitoring and quality improvement for health care. 


\section{Bibliography}

Arnold, A. M. and Kronmal, R. A. (2003). Multiple imputation of baseline data in the cardiovascular health study. AJE American Journal of Epidemiology, 157(1):74-84.

Berk, R. and MacDonald, J. (2008). Overdispersion and poisson regression. Journal of Quantitative Criminology, 269-284.

Combs, C. A., Murphy, E. L., and Laros, R. K. (1991). Factors associated with postpartum haemorrhage with vaginal birth. Obstetrics \& Gynecology, 66,3:353-356.

Coory, M., Duckett, D., and Sketcher-Baker, K. (2007). Using control charts to monitor quality of hospital care with administrative data. International Journal for Quality in Health Care Advance Access, pages 1-9.

Dover, D. and Schopflocher, D. (2011). Using funnel plots in public health surveillance. Population Health Metrics, 9:58.

Fox, J. (2008). Applied Regression Analysis and Generalized Linear Models. 'SAGE Publications, Inc.

Gale, C. P., Roberts, A. P., Batin, P. D., and Hall, A. S. (2006). Funnel plots, performance variation and the myocardial infraction national audit project 2003-2004. BMC Cardiovascular Disorders, 6:1471-226.

Gelman, A. and Hill, J. (2007). Data Analysis Using Regression and Multilevel/Hierarchical Models. Cambridge University Press, New York. 
Graham, P., Phil, H., Cumming, J., Raymont, A., and Finlayson, M. (2012). Variation in new zealand hospital outcomes: combining hierarchical bayesian modelling and propensity score methods for hospital performance comparisons. Health Service and Outcomes Research Methodology, 12:1-28.

Harrell, F. E. J. (2001). Regression Modeling Strategies. Springer-Verlag New York.

Harrison, K. \& Parke-Kivell, R. (1998). Management of postpartum haemorrhage. Prescriber Update, 16:4-9.

Jacobs, A. (2012). Causes and treatment of postpartum hemorrhage.

Knight, M., Callaghan, w. M., Berg, C., Alexander, S., ne Bouvier-Colle, M., Ford, J. B., Joseph, K. S., Lewis, G., Liston, R. M., Roberts, C. L., Oats, J., and Walker, J. (2009). Trends in postpartum hemorrhage in high resource countries: a review and recommendations from the international postpartum hemorrhage collaborative group. BMC Pregnancy and Childbirth, 9:55.

Little, R. J. A. and Rubin, D. B. (1989). Statistical Analysis with Missing Data. New York, NY: Wiley.

Marshall, C., Best, N., Bottle, A., and Aylin, P. (2004). Statistical issues in the prospective monitoring of health outcomes across multiple units. Journal of the Royal Statistical Society, Series A, 167:316-470.

McCormic, M. L., Sanghvi, H. C. G., Kinzie, B., and McIntosh, N. (2002). Preventing postpartum hemorrhage in low-resource settings. International Journal of Gynecology \& Obstetrics, 77:267-275.

Meng, X. L. (1994). Multiple-imputation inferences with uncongenial sources of input. Statistical Science, 9:538-573. 
Ministry of Health (2004). Report on maternity: Maternal and newborn information. Ministry of Health.

Ministry of Health (2012a). National minimum dataset (hospital events).

Ministry of Health (2012b). Nhi data dictionary v5.3.

Mousa, H. A. \& Alfirevic, Z. (2007). Treatment for primary postpartum haemorrhage. Cochrane Database of Systematic Review, 1:1-25.

National Womens Hospital (1996). National womens hospital annual report. Inhouse Publication.

Ohlssen, D. I., Sharplies, L. D., and J., S. D. (2007). A hierarchical modelling framework for identifying unusual performance in health care providers. Journal of Royal Statistical Society, 170:885-890.

Richardson, K., Blakely, T., Young, J., Graham, P., and Tobias, M. (2009). Do ethnic and socio-economic inequalities in mortality vary by region in new zealand? an application of hierarchical bayesian modelling. Social Science and Medicine, 69:1252-1260.

Rubin, D. B. (1976). Inference and missing data. Biometrika, 63:581-592.

Rubin, D. B. (1987). Multiple Imputation for Nonresponse in Surveys. John Wiley \& Sons, Inc.

Rubin, D. B. (1996). Multiple imputation after 18+ years. Journal of the American Statistical Association, 91:366-374.

Scheffer, J. (2002). Dealing with missing data. Res. Lett. Inf. Math. Sci, 3:153-160.

Schenker, N., Raghunathan, T. E., Chiu, P., Makuc, D. M., Zhang, G., and Cohen, A. J. (2006). Multiple imputation of missing income data in the national health interview survey. Journal of the American Statistical Association, 101(475):924-933. 
Spiegelhalter, D. J. (2005a). Funnel plots for comparing institutional performance. Statistics in Medicine, 24:1185-1202.

Spiegelhalter, D. J. (2005b). Handling over-dispersion of performance indicators. Quality Safety Health Care, 14:347-351.

Statistics New Zealand (2012). Statistics new zealand, www.stats.govt.nz.

Sterne, J. A. C. and Egger, M. (2001). Funnel plots for detecting bias in meta-analysis: Guidelines on choice of axis. Journal of Clinical Epidemiology, 54:1046-1055.

Taylor, K. L., Simpson, J. M., Roberts, C. L., Olive, E. C., and HendersonSmart, D. J. (2005). Risk of complications in a second pregnancy following caesarean section in the first pregnancy: a population-based study. Medical Journal of Australia, 183,10:515-519.

University of Virginia Health System (2010). High-risk pregnancy \& postpartum hemorrhage.

Waitemata District Health Board (2006). Waitemata district health board annual maternity \& gynecology services report. Website. 


\section{Appendix A}

\section{Glossary}

Assisted vaginal birth - A vaginal birth that requires assistance, for example, forceps.

Assisted vaginal birth, forceps - A vaginal birth requiring assistance with the use of a metallic obstetric instrument.

Assisted vaginal birth, vacuum extraction - A vaginal birth requiring assistance with the use of a suction cap that can be applied to the baby's head.

Assisted vaginal birth, vaginal breech birth - An vaginal birth requiring assistance because of the birth of a baby by the buttocks or lower limbs first rather than the head.

Birthweight - The first weight of the baby obtained after birth, usually to the nearest $5 \mathrm{~g}$.

Caesarean section - An operative birth through an abdominal incision.

Caesarean section, acute - also known as an Emergency Caesarean, a cae- 
sarean section performed urgently for clinical reasons once labour has started. For the health of the baby and/or mother.

Caesarean section, elective - A Caesarean section performed as planned.

DHB - District Health Board, An organisation established by section 19 of the NZ Public Health and Disability Act 2000.

Episiotomy - A small incision of the perineal tissue surrounding the vagina at the time of birth.

Gestation - The duration of pregnancy in completed weeks, calculated from the date of the first day of a woman's last menstrual period and her infant's date of birth, or derived from a clinical assessment during pregnancy, or derived from an examination of the infant after birth.

Induction - A medical intervention to stimulate the onset of labour.

Parity - The number of previous pregnancies a mother has had that have resulted in a live birth or still births.

Postpartum Haemorrhage (PPH) - Abnormal bleeding experienced by the mother soon after labour or childbirth.

Postpartum Haemorrhage, primary - A blood loss of more than $500 \mathrm{ml}$ from the genital tract within 24 hours of childbirth.

Postpartum Haemorrhage, secondary - A blood loss of more than $500 \mathrm{ml}$ from the genital tract after 24 hours of childbirth up until 6 weeks after delivery. 


\section{Appendix B}

\section{Imputation Regression Results}

\section{B.1 Gestation}

summary(lm.2)

Call:

$\operatorname{lm}($ formula $=$ gest $\sim$ mothers_age + ethnicgp_MAO + ethnicgp_PCI + ethnicgp_ASN + ethnicgp_OTH + ethnicgp_NA + as.factor(parity_bin.imp) + C_section + hosp_type + as.numeric(res_dep01) + sex + factor(dhb_birth $)+$ birth_weight.imp + pp_haem)

Residuals:

Min 1Q Median 3Q Max

$\begin{array}{lllll}-20.84 & -0.78 & 0.11 & 0.93 & 6.69\end{array}$

Signif. codes: $0 * * 0.001 * * 0.01 * 0.05 .0 .11$

Residual standard error: 1.503 on 54732 degrees of freedom (1045 observations deleted due to missingness)

Multiple R-squared: 0.3862, Adjusted R-squared: 0.3858

F-statistic: 1044 on 33 and $54732 \mathrm{DF}$, p-value: $<2.2 e-16$ 


\begin{tabular}{|c|c|c|c|c|c|}
\hline Coefficients: & Estimate & Std. Error & t value & $\operatorname{Pr}(>|t|)$ & \\
\hline (Intercept) & 32.88 & 0.07 & 496.18 & $<2 e-16$ & $* * *$ \\
\hline mothers_age & -0.01 & 0.00 & -9.47 & $<2 e-16$ & $* * *$ \\
\hline ethnicgp_MAO & -0.01 & 0.02 & -0.34 & 0.73 & \\
\hline ethnicgp_PCI & -0.21 & 0.02 & -8.32 & $<2 e-16$ & $* * *$ \\
\hline ethnicgp_ASN & 0.26 & 0.02 & 11.02 & $<2 e-16$ & $* * *$ \\
\hline ethnicgp_OTH & 0.15 & 0.04 & 3.70 & 0.00 & $* * *$ \\
\hline ethnicgp_NA & 0.09 & 0.08 & 1.12 & 0.26 & \\
\hline as.factor(parity_bin.imp)1 & -0.16 & 0.01 & -11.63 & $<2 e-16$ & $* * *$ \\
\hline C_section & -0.47 & 0.02 & -30.49 & $<2 e-16$ & $* * *$ \\
\hline hosp_type & 0.17 & 0.03 & 5.51 & 0.00 & $* * *$ \\
\hline as.numeric(res_dep01) & 0.00 & 0.00 & -0.08 & 0.93 & \\
\hline $\operatorname{sex}$ & -0.28 & 0.01 & -21.56 & $<2 e-16$ & $* * *$ \\
\hline factor(dhb_birth)10 & 0.01 & 0.05 & 0.26 & 0.79 & \\
\hline factor(dhb_birth)11 & -0.05 & 0.05 & -0.99 & 0.32 & \\
\hline factor(dhb_birth)12 & -0.13 & 0.06 & -2.06 & 0.04 & $*$ \\
\hline factor(dhb_birth)13 & -0.14 & 0.04 & -3.16 & 0.00 & $* *$ \\
\hline factor(dhb_birth)14 & -0.11 & 0.05 & -2.28 & 0.02 & $*$ \\
\hline factor(dhb_birth)15 & -0.12 & 0.08 & -1.53 & 0.13 & \\
\hline factor(dhb_birth)16 & -0.07 & 0.05 & -1.25 & 0.21 & \\
\hline factor(dhb_birth)17 & -0.28 & 0.09 & -3.11 & 0.00 & $* *$ \\
\hline factor(dhb_birth)18 & -0.06 & 0.04 & -1.37 & 0.17 & \\
\hline factor(dhb_birth)19 & -0.18 & 0.07 & -2.58 & 0.01 & $*$ \\
\hline factor(dhb_birth)2 & -0.14 & 0.04 & -3.53 & 0.00 & $* * *$ \\
\hline factor(dhb_birth)20 & -0.11 & 0.05 & -2.24 & 0.02 & $*$ \\
\hline factor(dhb_birth)21 & -0.08 & 0.05 & -1.43 & 0.15 & \\
\hline factor(dhb_birth)3 & -0.09 & 0.04 & -2.12 & 0.03 & $*$ \\
\hline factor(dhb_birth)4 & -0.11 & 0.04 & -2.78 & 0.01 & $* *$ \\
\hline factor(dhb_birth) 5 & -0.05 & 0.04 & -1.15 & 0.25 & \\
\hline factor(dhb_birth)6 & -0.06 & 0.05 & -1.05 & 0.29 & \\
\hline factor(dhb_birth)7 & -0.08 & 0.05 & -1.71 & 0.09 & . \\
\hline factor(dhb_birth) 8 & -0.16 & 0.07 & -2.32 & 0.02 & * \\
\hline factor(dhb_birth)9 & 0.05 & 0.05 & 0.94 & 0.35 & \\
\hline birth_weight.imp & 0.00 & 0.00 & 179.80 & $<2 e-16$ & $* * *$ \\
\hline pp_haem & -0.14 & 0.02 & -6.33 & 0.00 & $* * *$ \\
\hline
\end{tabular}

\section{B.2 Parity}

\section{Summary $(\operatorname{lm} .3)$}

Call:

$\operatorname{glm}($ formula $=$ as.factor $($ parity_bin $) \sim$ mothers_age + ethnicgp_MAO + ethnicgp_PCI + ethnicgp_ASN + ethnicgp_OTH + ethnicgp_NA + as.numeric(gest.imp) 
+ C_section + hosp_type + as.numeric(res_dep01) + sex + as.factor(dhb_birth)

+ p_haem + birth_weight.imp, family $=$ binomial(logit))

Deviance Residuals:

$\begin{array}{lcccc}\text { Min } & \text { 1Q } & \text { Median } & \text { 3Q } & \text { Max } \\ -2.71 & -0.83 & -0.50 & 0.93 & 2.89\end{array}$

Signif. codes: $0 * * 0.001 * * 0.01 * 0.05 \cdot 0.11$

(Dispersion parameter for binomial family taken to be 1)

Null deviance: 38552 on 29495 degrees of freedom Residual deviance: 31165 on 29462 degrees of freedom (26315 observations deleted due to missingness) AIC: 31233

Number of Fisher Scoring iterations: 4

\section{B.3 Birth Weight}

summary(lm.1)

Call:

$\operatorname{lm}($ formula $=$ birth_weight $\sim$ mothers_age + ethnicgp_MAO + ethnicgp_PCI

+ ethnicgp_ASN + ethnicgp_OTH + ethnicgp_NA + as.factor(parity_bin.imp)

+ C_section + hosp_type + as.numeric(res_dep01) + sex + factor(dhb_birth)

+ gest.imp + pp_haem)

Signif. codes: $0 * * * 0.001{ }^{* *} 0.01 * 0.05 \cdot 0.1 \quad 1$

Residual standard error: 454 on 55292 degrees of freedom 


$\begin{array}{lccccc}\text { Coefficients: } & \text { Estimate } & \text { Std. Error } & \mathrm{z} \text { value } & \operatorname{Pr}(>|z|) & \\ \text { (Intercept) } & -2.20 & 0.34 & -6.39 & 0.00 & * * * \\ \text { mothers_age } & 0.19 & 0.00 & 67.65 & <2 e-16 & * * * * \\ \text { ethnicgp_MAO } & 1.21 & 0.04 & 28.65 & <2 e-16 & * * * \\ \text { ethnicgp_PCI } & 1.12 & 0.05 & 20.55 & <2 e-16 & * * * \\ \text { ethnicgp_ASN } & 0.33 & 0.05 & 6.99 & 0.00 & * * * * \\ \text { ethnicgp_OTH } & 0.35 & 0.08 & 4.29 & 0.00 & * * * \\ \text { ethnicgp_NA } & -0.93 & 0.19 & -4.95 & 0.00 & * * * \\ \text { as.numeric(gest.imp) } & -0.16 & 0.01 & -17.33 & <2 e-16 & * * * * \\ \text { C_section } & -0.56 & 0.03 & -16.63 & <2 e-16 & * * * \\ \text { hosp_type } & 0.58 & 0.07 & 8.35 & <2 e-16 & * * * \\ \text { as.numeric(res_dep01) } & 0.05 & 0.01 & 9.65 & <2 e-16 & * * * \\ \text { sex } & -0.08 & 0.03 & -2.85 & 0.00 & * * \\ \text { as.factor(dhb_birth)10 } & -0.01 & 0.11 & -0.07 & 0.94 & \\ \text { as.factor(dhb_birth)11 } & 0.12 & 0.11 & 1.13 & 0.26 & \\ \text { as.factor(dhb_birth)12 } & -0.01 & 0.14 & -0.06 & 0.96 & \\ \text { as.factor(dhb_birth)13 } & -0.54 & 0.10 & -5.65 & 0.00 & * * * \\ \text { as.factor(dhb_birth)14 } & -0.11 & 0.11 & -1.07 & 0.29 & \\ \text { as.factor(dhb_birth)15 } & 0.18 & 0.18 & 1.01 & 0.31 & \\ \text { as.factor(dhb_birth)16 } & 0.09 & 0.11 & 0.81 & 0.42 & \\ \text { as.factor(dhb_birth)17 } & 0.39 & 0.19 & 2.05 & 0.04 & * \\ \text { as.factor(dhb_birth)18 } & -0.26 & 0.09 & -2.91 & 0.00 & * * * \\ \text { as.factor(dhb_birth)19 } & -0.38 & 0.15 & -2.49 & 0.01 & * \\ \text { as.factor(dhb_birth)2 } & -0.38 & 0.09 & -4.30 & 0.00 & * * * \\ \text { as.factor(dhb_birth)20 } & -0.17 & 0.11 & -1.52 & 0.13 & \\ \text { as.factor(dhb_birth)21 } & -0.23 & 0.12 & -1.88 & 0.06 & * \\ \text { as.factor(dhb_birth)3 } & -0.78 & 0.09 & -8.81 & <2 e-16 & * * * \\ \text { as.factor(dhb_birth)4 } & -0.15 & 0.09 & -1.74 & 0.08 & * \\ \text { as.factor(dhb_birth)5 } & -0.18 & 0.09 & -2.01 & 0.04 & * \\ \text { as.factor(dhb_birth)6 } & -0.13 & 0.12 & -1.14 & 0.25 & \\ \text { as.factor(dhb_birth)7 } & -0.02 & 0.10 & -0.19 & 0.85 & \\ \text { as.factor(dhb_birth)8 } & -0.05 & 0.16 & -0.29 & 0.77 & \\ \text { as.factor(dhb_birth)9 } & 0.10 & 0.10 & 1.00 & 0.32 & \\ \text { pp_haem } & -0.38 & 0.05 & -8.06 & 0.00 & * * * \\ \text { birth_weight.imp } & 0.00 & 0.00 & 21.29 & <2 e-16 & * * * \\ & & & & & \end{array}$

Residuals:

Min 1Q Median 3Q Max

$\begin{array}{lllll}-3092.7 & -299.4 & -14.3 & 281.6 & 3800.1\end{array}$

(485 observations deleted due to missingness)

Multiple R-squared: 0.3901, Adjusted R-squared: 0.3897

F-statistic: 1071 on 33 and $55292 \mathrm{DF}$, p-value: $<2.2 e-16$ 


$\begin{array}{lccccc}\text { Coefficients: } & \text { Estimate } & \text { Std. Error } & \mathrm{t} \text { value } & \operatorname{Pr}(>|t|) & \\ \text { (Intercept) } & -3850.39 & 43.71 & -88.09 & <2 e-16 & * * * \\ \text { mothers_age } & 4.02 & 0.34 & 11.77 & <2 e-16 & * * * \\ \text { ethnicgp_MAO } & -59.61 & 5.65 & -10.55 & <2 e-16 & * * * \\ \text { ethnicgp_PCI } & 85.62 & 7.43 & 11.52 & <2 e-16 & * * * \\ \text { ethnicgp_ASN } & -204.40 & 7.03 & -29.06 & <2 e-16 & * * * \\ \text { ethnicgp_OTH } & -80.25 & 12.44 & -6.45 & 0.00 & * * * \\ \text { ethnicgp_NA } & -42.51 & 23.08 & -1.84 & 0.07 & . \\ \text { as.factor(parity_bin.imp)1 } & 53.25 & 4.15 & 12.83 & <2 e-16 & * * * \\ \text { C_section } & 48.43 & 4.65 & 10.41 & <2 e-16 & * * * \\ \text { hosp_type } & 31.31 & 9.28 & 3.37 & 0.00 & * * * \\ \text { as.numeric(res_dep01) } & -2.25 & 0.78 & -2.87 & 0.00 & * * * \\ \text { sex } & 115.96 & 3.86 & 30.01 & <2 e-16 & * * * \\ \text { factor(dhb_birth)10 } & -0.31 & 16.15 & -0.02 & 0.98 & \\ \text { factor(dhb_birth)11 } & -0.84 & 14.76 & -0.06 & 0.95 & \\ \text { factor(dhb_birth)12 } & 20.63 & 19.42 & 1.06 & 0.29 & \\ \text { factor(dhb_birth)13 } & 27.71 & 13.46 & 2.06 & 0.04 & * \\ \text { factor(dhb_birth)14 } & 13.55 & 15.01 & 0.90 & 0.37 & \\ \text { factor(dhb_birth)15 } & 45.65 & 23.85 & 1.91 & 0.06 & . \\ \text { factor(dhb_birth)16 } & 16.28 & 16.04 & 1.02 & 0.31 & \\ \text { factor(dhb_birth)17 } & 32.51 & 26.95 & 1.21 & 0.23 & \\ \text { factor(dhb_birth)18 } & -13.71 & 12.41 & -1.11 & 0.27 & \\ \text { factor(dhb_birth)19 } & 41.17 & 21.55 & 1.91 & 0.06 & * \\ \text { factor(dhb_birth)2 } & 26.85 & 12.28 & 2.19 & 0.03 & * \\ \text { factor(dhb_birth)20 } & 1.87 & 15.15 & 0.12 & 0.90 & \\ \text { factor(dhb_birth)21 } & -15.64 & 16.52 & -0.95 & 0.34 & \\ \text { factor(dhb_birth)3 } & -1.10 & 12.42 & -0.09 & 0.93 & \\ \text { factor(dhb_birth)4 } & 8.30 & 12.06 & 0.69 & 0.49 & \\ \text { factor(dhb_birth)5 } & -3.91 & 12.77 & -0.31 & 0.76 & \\ \text { factor(dhb_birth)6 } & 10.33 & 16.19 & 0.64 & 0.52 & \\ \text { factor(dhb_birth)7 } & 1.18 & 13.86 & 0.09 & 0.93 & \\ \text { factor(dhb_birth)8 } & 17.65 & 20.75 & 0.85 & 0.40 & \\ \text { factor(dhb_birth)9 } & -14.91 & 14.83 & -1.01 & 0.31 & \\ \text { gest.imp } & 181.87 & 1.02 & 178.29 & <2 e-16 & * * * * \\ \text { pp_haem } & 110.30 & 6.67 & 16.53 & <2 e-16 & * * * \\ & & & & & \end{array}$




\section{Appendix C}

\section{Model Selection Results}

The follow SAS code is used for the logistic regression stepwise method:

PROC LOGISTIC DATA=data2;

CLASS induction (PARAM=EFFECT) ethnicgp (PARAM=EFFECT) res dep01 (PARAM=EFFECT);

MODEL pp_haem (Event $=$ ' 1 ') =gest imp mothers_age

parity_bin_imp C_section breech rem_placenta birth_weight_imp_log

episiotomy vacuum induction ethnicgp res_dep01 mothers_age*ethnicgp

mothers_age*birth_weight_imp_log birth_weight_imp_log*ethnicgp

mothers_age*parity_bin_imp parity_bin_imp*ethnicgp

parity_bin_imp*birth_weight_imp_log episiotomy*ethnicgp

birth_weight_imp_log*episiotomy mothers_age ${ }^{*} C_{-}$section

C_section*ethnicgp C_section*birth_weight_imp_log C_section*episiotomy

C_section*gest_imp breech*episiotomy gest_imp*breech C_section*breech

mothers_age*vacuum vacuum*ethnicgp birth_weight_imp_log*vacuum

episiotomy*vacuum gest imp*vacuum C section*vacuum breech*vacuum

mothers_age*induction birth_weight_imp_log*induction episiotomy*induction

gest_imp*induction C_section*induction breech*induction vacuum*induction

rem_placenta*ethnicgp C_section*rem_placenta rem_placenta*vacuum

gest_imp*birth_weight_imp_log induction*ethnicgp

/

SELECTION=STEPWISE

$\mathrm{SLE}=0.025$

SLS $=0.05$

LINK=LOGIT

RUN; 
The SAS output gives:

\begin{tabular}{|l|r|r|r|}
\hline \multicolumn{4}{|c|}{ Type 3 Analysis of Effects } \\
\hline Effect & DF & Square & Pr > ChiSq \\
\hline gest_imp & 1 & 96.72 & $<.0001$ \\
\hline parity_bin_imp & 1 & 6.18 & 0.01 \\
\hline C_section & 1 & 16.33 & $<.0001$ \\
\hline rem_placenta & 1 & 1727.54 & $<.0001$ \\
\hline birth_weight_imp_log & 1 & 40.26 & $<.0001$ \\
\hline episiotomy & 1 & 146.58 & $<.0001$ \\
\hline vacuum & 1 & 25.50 & $<.0001$ \\
\hline induction & 3 & 42.76 & $<.0001$ \\
\hline ethnicgp & 4 & 13.16 & 0.01 \\
\hline res_dep01 & 9 & 26.92 & 0.00 \\
\hline birth_weigh*ethnicgp & 4 & 14.97 & 0.00 \\
\hline parity_bi*birth_weig & 1 & 6.60 & 0.01 \\
\hline C_section*birth_weig & 1 & 10.43 & 0.00 \\
\hline vacuum*ethnicgp & 4 & 11.07 & 0.03 \\
\hline episiotomy*vacuum & 1 & 14.04 & 0.00 \\
\hline rem_placenta*vacuum & 1 & 18.90 & $<.0001$ \\
\hline gest_imp*birth_weigh & 1 & 92.60 & $<.0001$ \\
\hline induction*ethnicgp & 12 & 23.58 & 0.02 \\
\hline
\end{tabular}

\begin{tabular}{|l|r|r|r|}
\hline \multicolumn{4}{|c|}{ Odds Ratio Estimates } \\
\hline & $\begin{array}{r}\text { Point } \\
\text { Effect }\end{array}$ & \multicolumn{2}{|c|}{$\mathbf{9 5 \%}$ Wald } \\
\cline { 3 - 4 } & Estimate & Confidence Limits \\
\hline res_dep01 1 vs 9 & 0.77 & 0.66 & 0.89 \\
\hline res_dep01 10 vs 9 & 1.02 & 0.91 & 1.13 \\
\hline res_dep01 2 vs 9 & 0.89 & 0.77 & 1.02 \\
\hline res_dep01 3 vs 9 & 0.83 & 0.72 & 0.95 \\
\hline res_dep01 4 vs 9 & 0.90 & 0.79 & 1.02 \\
\hline res_dep01 5 vs 9 & 0.85 & 0.75 & 0.97 \\
\hline res_dep01 6 vs 9 & 1.00 & 0.88 & 1.12 \\
\hline res_dep01 7 vs 9 & 0.88 & 0.78 & 1.00 \\
\hline res_dep01 8 vs 9 & 0.88 & 0.79 & 0.99 \\
\hline
\end{tabular}

\begin{tabular}{|l|r|l|r|}
\hline \multicolumn{3}{|c|}{ Association of Predicted Probabilities and Observed } \\
\hline Percent Concordant & 68.3 & Somers' D & 0.38 \\
\hline Percent Discordant & 30.4 & Gamma & 0.38 \\
\hline Percent Tied & 1.3 & Tau-a & 0.07 \\
\hline Pairs & $2.62 \mathrm{E}+08$ & C & 0.69 \\
\hline
\end{tabular}


SAS output continued:

\begin{tabular}{|c|c|c|c|c|c|c|c|}
\hline \multicolumn{8}{|c|}{ Analys is of Maximum Likelihood Estimates } \\
\hline \multirow[b]{2}{*}{ Parameter } & & & \multirow[b]{2}{*}{ DF } & \multirow[b]{2}{*}{ Estimate } & \multirow{2}{*}{\begin{tabular}{|r|} 
Standard \\
Error \\
\end{tabular}} & \multirow{2}{*}{\begin{tabular}{r|} 
Wald \\
Square
\end{tabular}} & \multirow[b]{2}{*}{ Pr $>$ ChiSq } \\
\hline & & & & & & & \\
\hline Intercept & & & 1 & 19.02 & 3.32 & 32.78 & $<.0001$ \\
\hline gest_imp & & & 1 & -0.85 & 0.09 & 96.72 & $<.0001$ \\
\hline parity_bin_imp & & & 1 & 2.71 & 1.09 & 6.18 & 0.01 \\
\hline C_section & & & 1 & 4.32 & 1.07 & 16.33 & $<.0001$ \\
\hline rem_placenta & & & 1 & 3.70 & 0.09 & 1727.54 & $<.0001$ \\
\hline birth_weight_imp_log & & & 1 & -2.77 & 0.44 & 40.26 & $<.0001$ \\
\hline episiotomy & & & 1 & 0.69 & 0.06 & 146.58 & $<.0001$ \\
\hline vacuum & & & 1 & 0.57 & 0.11 & 25.50 & $<.0001$ \\
\hline induction & $\mathrm{BN}$ & & 1 & -0.16 & 0.06 & 7.58 & 0.01 \\
\hline induction & $\mathrm{M}$ & & 1 & -0.16 & 0.08 & 3.61 & 0.06 \\
\hline induction & MS & & 1 & 0.31 & 0.07 & 18.91 & $<.0001$ \\
\hline ethnicgp & 11 & & 1 & 1.19 & 0.96 & 1.52 & 0.22 \\
\hline ethnicgp & 12 & & 1 & -3.09 & 1.26 & 6.02 & 0.01 \\
\hline ethnicgp & 13 & & 1 & -2.47 & 1.40 & 3.11 & 0.08 \\
\hline ethnicgp & 14 & & 1 & 1.59 & 1.43 & 1.25 & 0.26 \\
\hline res_dep01 & 1 & & 1 & -0.16 & 0.06 & 7.58 & 0.01 \\
\hline res_dep01 & 10 & & 1 & 0.12 & 0.04 & 8.50 & 0.00 \\
\hline res_dep01 & 2 & & 1 & -0.01 & 0.05 & 0.03 & 0.85 \\
\hline res_dep01 & 3 & & 1 & -0.08 & 0.05 & 2.03 & 0.15 \\
\hline res_dep01 & 4 & & 1 & 0.00 & 0.05 & 0.00 & 0.99 \\
\hline res_dep01 & 5 & & 1 & -0.05 & 0.05 & 1.17 & 0.28 \\
\hline res_dep01 & 6 & & 1 & 0.10 & 0.04 & 5.38 & 0.02 \\
\hline res_dep01 & 7 & & 1 & -0.02 & 0.05 & 0.16 & 0.69 \\
\hline res_dep01 & 8 & & 1 & -0.02 & 0.04 & 0.17 & 0.68 \\
\hline birth_weigh*ethnicgp & 11 & & 1 & -0.18 & 0.12 & 2.39 & 0.12 \\
\hline birth_weigh*ethnicgp & 12 & & 1 & 0.37 & 0.16 & 5.52 & 0.02 \\
\hline birth_weigh*ethnicgp & 13 & & 1 & 0.35 & 0.17 & 4.13 & 0.04 \\
\hline birth_weigh*ethnicgp & 14 & & 1 & -0.20 & 0.18 & 1.30 & 0.25 \\
\hline parity_bibirth_weig & & & 1 & -0.35 & 0.13 & 6.60 & 0.01 \\
\hline C_section*birth_weig & & & 1 & -0.43 & 0.13 & 10.43 & 0.00 \\
\hline vacuum*ethnicgp & 11 & & 1 & 0.19 & 0.11 & 3.14 & 0.08 \\
\hline vacuum*ethnicgp & 12 & & 1 & -0.02 & 0.16 & 0.02 & 0.90 \\
\hline vacuumethnicgp & 13 & & 1 & -0.30 & 0.19 & 2.53 & 0.11 \\
\hline vacuum*ethnicgp & 14 & & 1 & 0.40 & 0.14 & 8.42 & 0.00 \\
\hline episiotomy ${ }^{*}$ vacuum & & & 1 & -0.44 & 0.12 & 14.04 & 0.00 \\
\hline rem_placenta*vacuum & & & 1 & -1.06 & 0.24 & 18.90 & $<.0001$ \\
\hline gest_imp*birth_weigh & & & 1 & 0.11 & 0.01 & 92.60 & $<.0001$ \\
\hline induction*ethnicgp & $\mathrm{BN}$ & 11 & 1 & 0.05 & 0.07 & 0.61 & 0.44 \\
\hline induction*ethnicgp & $\mathrm{BN}$ & 12 & 1 & 0.17 & 0.09 & 3.55 & 0.06 \\
\hline induction*ethnicgp & $\mathrm{BN}$ & 13 & 1 & 0.01 & 0.10 & 0.00 & 0.95 \\
\hline induction*ethnicgp & $\mathrm{BN}$ & 14 & 1 & 0.05 & 0.12 & 0.15 & 0.70 \\
\hline induction*ethnicgp & $M$ & 11 & 1 & 0.24 & 0.10 & 5.69 & 0.02 \\
\hline induction*ethnicgp & $\mathrm{M}$ & 12 & 1 & -0.08 & 0.14 & 0.34 & 0.56 \\
\hline induction*ethnicgp & $\mathrm{M}$ & 13 & 1 & -0.13 & 0.15 & 0.81 & 0.37 \\
\hline induction*ethnicgp & $\mathrm{M}$ & 14 & 1 & 0.22 & 0.16 & 1.83 & 0.18 \\
\hline induction*ethnicgp & MS & 11 & 1 & -0.24 & 0.09 & 7.62 & 0.01 \\
\hline induction*ethnicgp & MS & 12 & 1 & 0.02 & 0.11 & 0.04 & 0.84 \\
\hline induction*ethnicgp & MS & 13 & 1 & -0.01 & 0.12 & 0.01 & 0.94 \\
\hline induction*ethnicgp & MS & 14 & 1 & 0.12 & 0.14 & 0.75 & 0.39 \\
\hline
\end{tabular}


Goodness of Fit

\begin{tabular}{|r|r|r|r|r|r|}
\hline \multicolumn{6}{|c|}{ Partition for the Hosmer and Lemeshow Test } \\
\hline & & \multicolumn{2}{|c|}{ pp_haem =1 } & \multicolumn{2}{|c|}{ pp_haem = 0 } \\
\cline { 3 - 6 } Group & Total & Observed & Expected & Observed & Expected \\
\hline $\mathbf{1}$ & 5561 & 225 & 203.89 & 5336 & 5357.11 \\
\hline $\mathbf{2}$ & 5537 & 209 & 258.62 & 5328 & 5278.38 \\
\hline $\mathbf{3}$ & 5550 & 272 & 290.11 & 5278 & 5259.89 \\
\hline $\mathbf{4}$ & 5539 & 311 & 324.83 & 5228 & 5214.17 \\
\hline $\mathbf{5}$ & 5538 & 384 & 373.8 & 5154 & 5164.2 \\
\hline $\mathbf{6}$ & 5535 & 468 & 455.62 & 5067 & 5079.38 \\
\hline $\mathbf{7}$ & 5538 & 538 & 540.55 & 5000 & 4997.45 \\
\hline $\mathbf{8}$ & 5539 & 573 & 617.09 & 4966 & 4921.91 \\
\hline $\mathbf{9}$ & 5534 & 775 & 719.34 & 4759 & 4814.66 \\
\hline $\mathbf{1 0}$ & 5504 & 1459 & 1430.13 & 4045 & 4073.87 \\
\hline
\end{tabular}

\begin{tabular}{|r|r|r|}
\hline \multicolumn{2}{|c|}{$\begin{array}{c}\text { Hosmer and Lemeshow } \\
\text { Goodness-of-Fit }\end{array}$} \\
\hline \multicolumn{3}{|c|}{ Test } \\
\hline $\begin{array}{r}\text { Chi- } \\
\text { Square }\end{array}$ & DF & Pr > ChiSq \\
\hline 24.0328 & 8 & 0.0023 \\
\hline
\end{tabular}

Deviance and Pearson Goodness-of-Fit Statistics

\begin{tabular}{|l|r|r|r|r|}
\hline Criterion & Value & DF & Value/DF & $\mathbf{q}$ \\
\hline Deviance & 12978.85 & $1.60 \mathrm{E}+04$ & 0.8128 & 1 \\
\hline Pearson & 18028.06 & $1.60 \mathrm{E}+04$ & 1.129 & $<.0001$ \\
\hline
\end{tabular}

\begin{tabular}{|l|r|r|}
\hline \multicolumn{3}{|c|}{ Model Fit Statistics } \\
\hline \multirow{4}{*}{ Criterion } & Intercept & Intercept \\
\cline { 2 - 3 } & Only & and \\
\cline { 2 - 3 } AIC & & Covariates \\
\hline SC & 34561.96 & 31593.434 \\
\hline $\mathbf{- 2}$ Log L & 34559.962 & 31495.434 \\
\hline
\end{tabular}

\begin{tabular}{|l|l|l|l|}
\hline R-Square & 0.0538 & Max-rescal & 0.116 \\
\hline
\end{tabular}

\begin{tabular}{|l|r|r|r|}
\hline \multicolumn{4}{|c|}{ Testing Global Null Hypothesis: BETA=0 } \\
\hline Test & $\begin{array}{r}\text { Chi- } \\
\text { Square }\end{array}$ & DF & Pr $>$ ChiSq \\
\hline Likelihood & 3064.527 & 48 & $<.0001$ \\
\hline Score & 4814.224 & 48 & $<.0001$ \\
\hline Wald & 2676.975 & 48 & $<.0001$ \\
\hline
\end{tabular}




\section{C.1 Regression results for Adjusted Plot}

The follow SAS code is used for the logistic regression method:

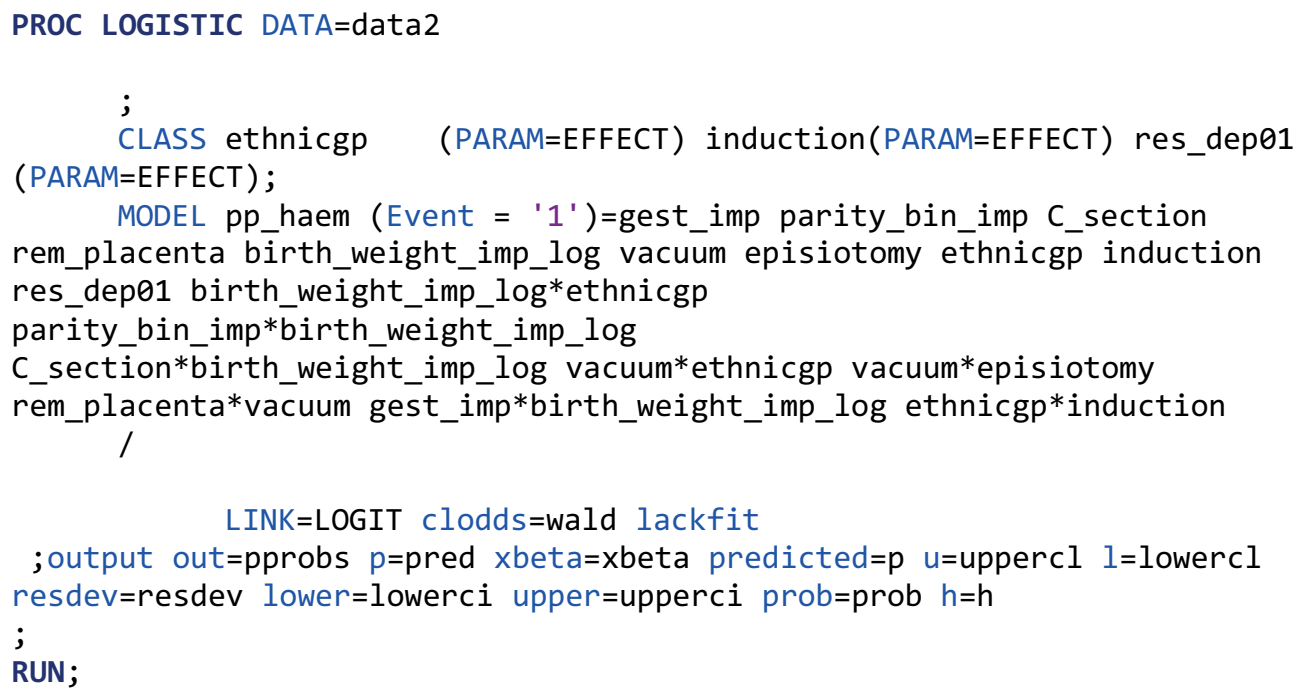

The SAS output gives:

\begin{tabular}{|l|c|c|}
\hline \multicolumn{3}{|c|}{ Model Fit Statistics } \\
\hline \multirow{3}{*}{ Criterion } & Intercept & Intercept \\
\cline { 2 - 3 } & Only & and \\
\cline { 2 - 3 } & & Covariates \\
\hline AIC & 34561.96 & 31593.434 \\
\hline SC & 34570.88 & 32030.607 \\
\hline-2 Log L & 34559.962 & 31495.434 \\
\hline
\end{tabular}

Testing Global Null Hypothes is: BETA=0

\begin{tabular}{|l|c|r|r|}
\hline \multicolumn{1}{|c|}{ Test } & $\begin{array}{c}\text { Chi- } \\
\text { Square }\end{array}$ & DF & Pr $>$ ChiSq \\
\hline Likelihood & 3064.527 & 48 & $<.0001$ \\
\hline Score & 4814.224 & 48 & $<.0001$ \\
\hline Wald & 2676.975 & 48 & $<.0001$ \\
\hline
\end{tabular}




\begin{tabular}{|l|r|l|r|}
\hline \multicolumn{3}{|c|}{ Responses } \\
\hline Percent Concordant & 68.3 & Somers' D & 0.378 \\
\hline Percent Discordant & 30.4 & Gamma & 0.383 \\
\hline Percent Tied & 1.3 & Tau-a & 0.065 \\
\hline Pairs & 261539454 & C & 0.689 \\
\hline
\end{tabular}

\begin{tabular}{|c|r|r|}
\hline \multicolumn{3}{|c|}{ Hosmer and Lemeshow Goodness-of-Fit } \\
\hline \multicolumn{3}{|c|}{ Test } \\
\hline Chi-Square & DF & Pr $>$ ChiSq \\
\hline 24.0328 & 8 & 0.0023 \\
\hline
\end{tabular}

\begin{tabular}{|l|r|r|r|}
\hline \multicolumn{3}{|c|}{ Type 3 Analysis of Effects } \\
\hline Effect & DF & $\begin{array}{c}\text { Wald Chi- } \\
\text { Square }\end{array}$ & Pr $>$ ChiSq \\
\hline gest_imp & 1 & 96.7217 & $<.0001$ \\
\hline parity_bin_imp & 1 & 6.1813 & 0.0129 \\
\hline C_section & 1 & 16.3346 & $<.0001$ \\
\hline rem_placenta & 1 & 1727.541 & $<.0001$ \\
\hline birth_weight_imp_log & 1 & 40.2573 & $<.0001$ \\
\hline vacuum & 1 & 25.5044 & $<.0001$ \\
\hline episiotomy & 1 & 146.5798 & $<.0001$ \\
\hline ethnicgp & 4 & 13.1602 & 0.0105 \\
\hline induction & 3 & 42.7636 & $<.0001$ \\
\hline res_dep01 & 9 & 26.9201 & 0.0014 \\
\hline birth_weigh*ethnicgp & 4 & 14.9698 & 0.0048 \\
\hline parity_bi*birth_weig & 1 & 6.6014 & 0.0102 \\
\hline C_section*birth_weig & 1 & 10.4318 & 0.0012 \\
\hline vacuum*ethnicgp & 4 & 11.0685 & 0.0258 \\
\hline vacuum ${ }^{*}$ episiotomy & 1 & 14.0375 & 0.0002 \\
\hline rem_placenta*vacuum & 1 & 18.9003 & $<.0001$ \\
\hline gest_imp*birth_weigh & 1 & 92.6004 & $<.0001$ \\
\hline ethnicgp*induction & 12 & 23.5845 & 0.0232 \\
\hline
\end{tabular}




\begin{tabular}{|c|c|c|c|c|c|c|c|}
\hline \multicolumn{8}{|c|}{ Analysis of Maximum Likelihood Estimates } \\
\hline Parameter & & & DF & Estimate & $\begin{array}{c}\text { Standard } \\
\text { Error }\end{array}$ & \begin{tabular}{|c|} 
Wald Chi- \\
Square
\end{tabular} & $\mathrm{Pr}>\mathrm{ChiSq}$ \\
\hline Intercept & & & 1 & 19.02 & 3.32 & 32.78 & $<.0001$ \\
\hline gest_imp & & & 1 & -0.85 & 0.09 & 96.72 & $<.0001$ \\
\hline parity_bin_imp & & & 1 & 2.71 & 1.09 & 6.18 & 0.01 \\
\hline C_section & & & 1 & 4.32 & 1.07 & 16.33 & $<.0001$ \\
\hline rem_placenta & & & 1 & 3.70 & 0.09 & 1727.54 & $<.0001$ \\
\hline birth_weight_imp_log & & & 1 & -2.77 & 0.44 & 40.26 & $<.0001$ \\
\hline vacuum & & & 1 & 0.57 & 0.11 & 25.50 & $<.0001$ \\
\hline episiotomy & & & 1 & 0.69 & 0.06 & 146.58 & $<.0001$ \\
\hline ethnicgp & 11 & & 1 & 1.19 & 0.96 & 1.52 & 0.22 \\
\hline ethnicgp & 12 & & 1 & -3.09 & 1.26 & 6.02 & 0.01 \\
\hline ethnicgp & 13 & & 1 & -2.47 & 1.40 & 3.11 & 0.08 \\
\hline ethnicgp & 14 & & 1 & 1.59 & 1.43 & 1.25 & 0.26 \\
\hline induction & $\mathrm{BN}$ & & 1 & -0.16 & 0.06 & 7.58 & 0.01 \\
\hline induction & $\mathrm{M}$ & & 1 & -0.16 & 0.08 & 3.61 & 0.06 \\
\hline induction & $\mathrm{MS}$ & & 1 & 0.31 & 0.07 & 18.91 & $<.0001$ \\
\hline res_dep01 & 1 & & 1 & -0.16 & 0.06 & 7.58 & 0.01 \\
\hline res_dep01 & 10 & & 1 & 0.12 & 0.04 & 8.50 & 0.00 \\
\hline res_dep01 & 2 & & 1 & -0.01 & 0.05 & 0.03 & 0.85 \\
\hline res_dep01 & 3 & & 1 & -0.08 & 0.05 & 2.03 & 0.15 \\
\hline res_dep01 & 4 & & 1 & 0.00 & 0.05 & 0.00 & 0.99 \\
\hline res_dep01 & 5 & & 1 & -0.05 & 0.05 & 1.17 & 0.28 \\
\hline res_dep01 & 6 & & 1 & 0.10 & 0.04 & 5.38 & 0.02 \\
\hline res_dep01 & 7 & & 1 & -0.02 & 0.05 & 0.16 & 0.69 \\
\hline res_dep01 & 8 & & 1 & -0.02 & 0.04 & 0.17 & 0.68 \\
\hline birth_weigh*ethnicgp & 11 & & 1 & -0.18 & 0.12 & 2.39 & 0.12 \\
\hline birth_weigh*ethnicgp & 12 & & 1 & 0.37 & 0.16 & 5.52 & 0.02 \\
\hline birth_weigh*ethnicgp & 13 & & 1 & 0.35 & 0.17 & 4.13 & 0.04 \\
\hline birth_weigh*ethnicgp & 14 & & 1 & -0.20 & 0.18 & 1.30 & 0.25 \\
\hline parity_bi*birth_weig & & & 1 & -0.35 & 0.13 & 6.60 & 0.01 \\
\hline C_section*birth_weig & & & 1 & -0.43 & 0.13 & 10.43 & 0.00 \\
\hline vacuum*ethnicgp & 11 & & 1 & 0.19 & 0.11 & 3.14 & 0.08 \\
\hline vacuum*ethnicgp & 12 & & 1 & -0.02 & 0.16 & 0.02 & 0.90 \\
\hline vacuum*ethnicgp & 13 & & 1 & -0.30 & 0.19 & 2.53 & 0.11 \\
\hline vacuum*ethnicgp & 14 & & 1 & 0.40 & 0.14 & 8.42 & 0.00 \\
\hline vacuum*episiotomy & & & 1 & -0.44 & 0.12 & 14.04 & 0.00 \\
\hline rem_placenta*vacuum & & & 1 & -1.06 & 0.24 & 18.90 & $<.0001$ \\
\hline gest_imp*birth_weigh & & & 1 & 0.11 & 0.01 & 92.60 & $<.0001$ \\
\hline ethnicgp*induction & 11 & $\mathrm{BN}$ & 1 & 0.05 & 0.07 & 0.61 & 0.44 \\
\hline ethnicgp*induction & 11 & $M$ & 1 & 0.24 & 0.10 & 5.69 & 0.02 \\
\hline ethnicgp*induction & 11 & MS & 1 & -0.24 & 0.09 & 7.62 & 0.01 \\
\hline ethnicgp*induction & 12 & $\mathrm{BN}$ & 1 & 0.17 & 0.09 & 3.55 & 0.06 \\
\hline ethnicgp*induction & 12 & $M$ & 1 & -0.08 & 0.14 & 0.34 & 0.56 \\
\hline ethnicgp*induction & 12 & MS & 1 & 0.02 & 0.11 & 0.04 & 0.84 \\
\hline ethnicgp*induction & 13 & $\mathrm{BN}$ & 1 & 0.01 & 0.10 & 0.00 & 0.95 \\
\hline ethnicgp*induction & 13 & $\mathrm{M}$ & 1 & -0.13 & 0.15 & 0.81 & 0.37 \\
\hline ethnicgp*induction & 13 & $\mathrm{MS}$ & 1 & -0.01 & 0.12 & 0.01 & 0.94 \\
\hline ethnicgp*induction & 14 & $\mathrm{BN}$ & 1 & 0.05 & 0.12 & 0.15 & 0.70 \\
\hline ethnicgp*induction & 14 & $\mathrm{M}$ & 1 & 0.22 & 0.16 & 1.83 & 0.18 \\
\hline ethnicgp*induction & 14 & MS & 1 & 0.12 & 0.14 & 0.75 & 0.39 \\
\hline
\end{tabular}

